\title{
Poset Topology: Tools and Applications
}

\author{
Michelle L. Wachs
}




\section{Contents}

Poset Topology: Tools and Applications 1

Introduction 3

Lecture 1. Basic definitions, results, and examples 5

1.1. Order complexes and face posets 5

1.2. The Möbius function 9

1.3. Hyperplane and subspace arrangements 11

1.4. Some connections with graphs, groups and lattices 16

$\begin{array}{ll}\text { 1.5. Poset homology and cohomology } & 17\end{array}$

1.6. Top cohomology of the partition lattice 19

Lecture 2. Group actions on posets 23

2.1. Group representations 23

2.2. Representations of the symmetric group 25

2.3. Group actions on poset (co)homology 30

2.4. Symmetric functions, plethysm, and wreath product modules 32

Lecture 3. Shellability and edge labelings $\quad 41$

3.1. Shellable simplicial complexes 41

3.2. Lexicographic shellability 45

3.3. CL-shellability and Coxeter groups $\quad 57$

3.4. Rank selection $\quad 62$

$\begin{array}{ll}\text { Lecture 4. Recursive techniques } & 67\end{array}$

4.1. Cohen-Macaulay complexes 67

4.2. Recursive atom orderings $\quad 71$

4.3. More examples $\quad 73$

4.4. The Whitney homology technique 77

4.5. Bases for the restricted block size partition posets 82

4.6. Fixed point Möbius invariant $\quad 90$

Lecture 5. Poset operations and maps 91

5.1. Operations: Alexander duality and direct product 91

5.2. Quillen fiber lemma $\quad 95$

5.3. General poset fiber theorems 100

5.4. Fiber theorems and subspace arrangements 103

5.5. Inflations of simplicial complexes 105

$\begin{array}{ll}\text { Bibliography } & 109\end{array}$ 
IAS/Park City Mathematics Series

Volume 00, 2004

\title{
Poset Topology: Tools and Applications
}

\author{
Michelle L. Wachs
}

\section{Introduction}

The theory of poset topology evolved from the seminal 1964 paper of Gian-Carlo Rota on the Möbius function of a partially ordered set. This theory provides a deep and fundamental link between combinatorics and other branches of mathematics. Early impetus for this theory came from diverse fields such as

- commutative algebra (Stanley's 1975 proof of the upper bound conjecture)

- group theory (the work of Brown (1974) and Quillen (1978) on p-subgroup posets)

- combinatorics (Björner's 1980 paper on poset shellability)

- representation theory (Stanley's 1982 paper on group actions on the homology of posets)

- topology (the Orlik-Solomon theory of hyperplane arrangements (1980))

- complexity theory (the 1984 paper of Kahn, Saks, and Sturtevant on the evasiveness conjecture).

Later developments have kept the theory vital. I mention just a few examples: Goresky-MacPherson formula for subspace arrangements, Björner-Lovász-Yao complexity theory results, Björner-Wachs extension of shellability to nonpure complexes, Forman's discrete version of Morse theory, and Vassiliev's work on knot invariants and graph connectivity.

So, what is poset topology? By the topology of a partially ordered set (poset) we mean the topology of a certain simplicial complex associated with the poset, called the order complex of the poset. In these lectures I will present some of the techniques that have been developed over the years to study the topology of a poset, and discuss some of the applications of poset topology to the fields mentioned above as well as to other fields. In particular, I will discuss tools for computing homotopy type and (co)homology of posets, with an emphasis on group equivariant (co)homology. Although posets and simplicial complexes can be viewed as essentially the same topological object, we will narrow our focus, for the most part,

Department of Mathematics, University of Miami, Coral Gables, Fl 33124.

E-mail address: wachs@math.miami.edu.

This work was partially supported by NSF grant DMS 0302310 . 
to tools that were developed specifically for posets; for example, lexicographical shellability, recursive atom orderings, Whitney homology techniques, (co)homology bases/generating set techniques, and fiber theorems.

Research in poset topology is very much driven by the study of concrete examples that arise in various contexts both inside and outside of combinatorics. These examples often turn out to have a rich and interesting topological structure, whose analysis leads to the development of new techniques in poset topology. These lecture notes are organized according to techniques rather than applications. A recurring theme is the use of original examples in demonstrating a technique, where by original example I mean the example that led to the development of the technique in the first place. More recent examples will be discussed as well.

With regard to the choice of topics, I was primarily motivated by my own research interests and the desire to provide the students at the PCMI graduate school with concrete skills in this subject. Due to space and time constraints and my decision to focus on techniques specific to posets, there are a number of very important tools for general simplicial complexes that I have only been able to mention in passing (or not at all). I point out, in particular, discrete Morse theory (which is a major part of the lecture series of Robin Forman, its originator) and basic techniques from algebraic topology such as long exact sequences and spectral sequences. For further techniques and applications, still of current interest, we strongly recommend the influential 1995 book chapter of Anders Björner [29].

The exercises vary in difficulty and are there to reinforce and supplement the material treated in these notes. There are many open problems (simply referred to as problems) and conjectures sprinkled throughout the text.

I would like to thank the organizers (Ezra Miller, Vic Reiner and Bernd Sturmfels) of the 2004 PCMI Graduate Summer School for inviting me to deliver these lectures. I am very grateful to Vic Reiner for his encouragement and support. I would also like to thank Tricia Hersh for the help and support she provided as my overqualified teaching assistant. Finally, I would like to express my gratitude to the graduate students at the summer school for their interest and inspiration. 


\section{LECTURE 1 \\ Basic definitions, results, and examples}

\subsection{Order complexes and face posets}

We begin by defining the order complex of a poset and the face poset of a simplicial complex. These constructions enable us to view posets and simplicial complexes as essentially the same topological object. We shall assume throughout these lectures that all posets and simplicial complexes are finite, unless otherwise stated.

An abstract simplicial complex $\Delta$ on finite vertex set $V$ is a nonempty collection of subsets of $V$ such that

- $\{v\} \in \Delta$ for all $v \in V$

- if $G \in \Delta$ and $F \subseteq G$ then $F \in \Delta$.

The elements of $\Delta$ are called faces (or simplices) of $\Delta$ and the maximal faces are called facets. We say that a face $F$ has dimension $d$ and write $\operatorname{dim} F=d$ if $d=|F|-1$. Faces of dimension $d$ are referred to as $d$-faces. The dimension $\operatorname{dim} \Delta$ of $\Delta$ is defined to be $\max _{F \in \Delta} \operatorname{dim} F$. We also allow the (-1)-dimensional complex $\{\emptyset\}$, which we refer to as the empty simplicial complex. It will be convenient to refer to the empty set $\emptyset$, as the degenerate empty complex and say that it has dimension -2 , even though we don't really consider it to be a simplicial complex. If all facets of $\Delta$ have the same dimension then $\Delta$ is said to be pure.

A $d$-dimensional geometric simplex in $\mathbb{R}^{n}$ is defined to be the convex hull of $d+1$ affinely independent points in $\mathbb{R}^{n}$ called vertices. The convex hull of any subset of the vertices is called a face of the geometric simplex. A geometric simplicial complex $K$ in $\mathbb{R}^{n}$ is a nonempty collection of geometric simplices in $\mathbb{R}^{n}$ such that

- Every face of a simplex in $K$ is in $K$.

- The intersection of any two simplices of $K$ is a face of both of them.

From a geometric simplicial complex $K$, one gets an abstract simplicial complex $\Delta(K)$ by letting the faces of $\Delta(K)$ be the vertex sets of the simplices of $K$. Every abstract simplicial complex $\Delta$ can be obtained in this way, i.e., there is a geometric simplicial complex $K$ such that $\Delta(K)=\Delta$. Although $K$ is not unique, the underlying topological space, obtained by taking the union of the simplices of $K$ under the usual topology on $\mathbb{R}^{n}$, is unique up to homeomorphism. We refer to this space as the geometric realization of $\Delta$ and denote it by $\|\Delta\|$. We will usually 


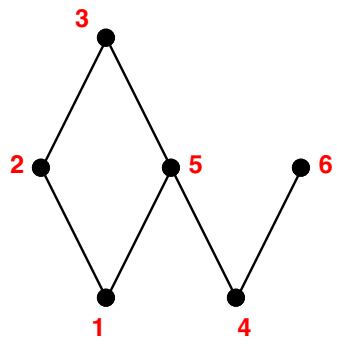

$\mathbf{P}$

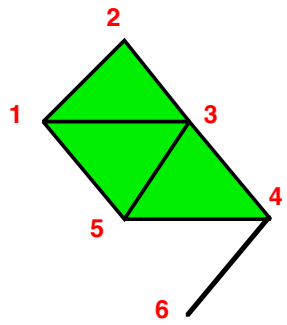

$\Delta(\mathrm{P})$

Figure 1.1.1. Order complex of a poset

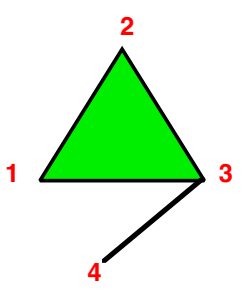

$\Delta$

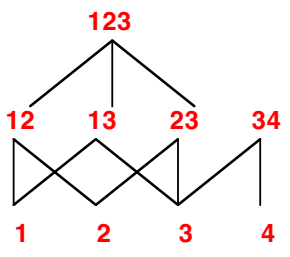

$\mathbf{P}(\Delta)$

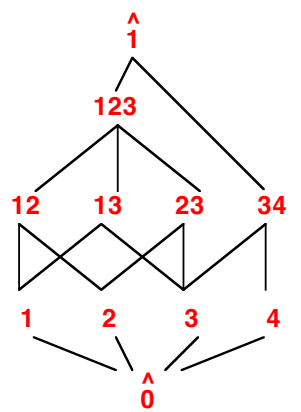

$\mathrm{L}(\Delta)$

Figure 1.1.2. Face poset and face lattice of a simplicial complex

drop the \|\| and let $\Delta$ denote an abstract simplicial complex as well as its geometric realization.

To every poset $P$, one can associate an abstract simplicial complex $\Delta(P)$ called the order complex of $P$. The vertices of $\Delta(P)$ are the elements of $P$ and the faces of $\Delta(P)$ are the chains (i.e., totally ordered subsets) of $P$. (The order complex of the empty poset is the empty simplicial complex $\{\emptyset\}$.) For example, the Hasse diagram of a poset $P$ and the geometric realization of its order complex are given in Figure 1.1.1.

To every simplicial complex $\Delta$, one can associate a poset $P(\Delta)$ called the face poset of $\Delta$, which is defined to be the poset of nonempty faces ordered by inclusion. The face lattice $L(\Delta)$ is $P(\Delta)$ with a smallest element $\hat{0}$ and a largest element $\hat{1}$ attached. An example is given in Figure 1.1.2. 


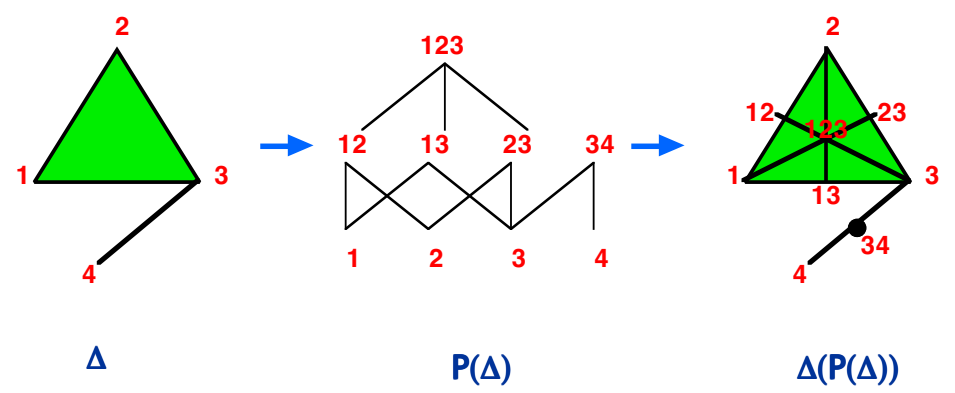

Figure 1.1.3. Barycentric subdivision

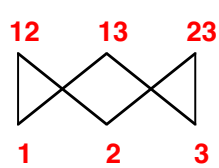

$\overline{B_{3}}$

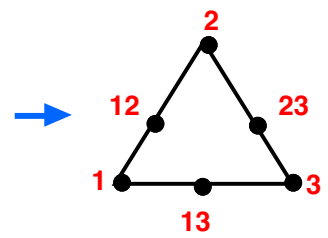

$\Delta\left(\overline{B_{3}}\right)$

Figure 1.1.4. Order complex of the subset lattice (Boolean algebra)

If we start with a simplicial complex $\Delta$, take its face poset $P(\Delta)$, and then take the order complex $\Delta(P(\Delta))$, we get a simplicial complex known as the barycentric subdivision of $\Delta$; see Figure 1.1.3. The geometric realizations are always homeomorphic,

$$
\Delta \cong \Delta(P(\Delta))
$$

When we attribute a topological property to a poset, we mean that the geometric realization of the order complex of the poset has that property. For instance, if we say that the poset $P$ is homeomorphic to the $n$-sphere $\mathbb{S}^{n}$ we mean that $\|\Delta(P)\|$ is homeomorphic to $\mathbb{S}^{n}$.

Example 1.1.1. The Boolean algebra. Let $B_{n}$ denote the lattice of subsets of $[n]:=\{1,2, \ldots, n\}$ ordered by containment, and let $\bar{B}_{n}:=B_{n}-\{\emptyset,[n]\}$. Then

$$
\bar{B}_{n} \cong \mathbb{S}^{n-2}
$$

because $\Delta\left(\bar{B}_{n}\right)$ is the barycentric subdivision of the boundary of the $(n-1)$-simplex. See Figure 1.1.4.

We now review some basic poset terminology. An $m$-chain of a poset $P$ is a totally ordered subset $c=\left\{x_{1}<x_{2}<\cdots<x_{m+1}\right\}$ of $P$. We say the length $l(c)$ of $c$ is $m$. We consider the empty chain to be a $(-1)$-chain. The length $l(P)$ of $P$ is 
defined to be

$$
l(P):=\max \{l(c): c \text { is a chain of } P\} .
$$

Thus, $l(P)=\operatorname{dim} \Delta(P)$ and $l(P(\Delta))=\operatorname{dim} \Delta$.

A chain of $P$ is said to be maximal if it is inclusionwise maximal. Thus, the set $\mathcal{M}(P)$ of maximal chains of $P$ is the set of facets of $\Delta(P)$. A poset $P$ is said to be pure (also known as ranked or graded) if all maximal chains have the same length. Thus, $P$ is pure if and only if $\Delta(P)$ is pure. Also a simplicial complex $\Delta$ is pure if and only if its face poset $P(\Delta)$ is pure. The posets and simplicial complexes of Figures 1.1.1 and 1.1.2 are all nonpure, while the poset and simplicial complex of Figure 1.1.4 are both pure.

For $x \leq y$ in $P$, let $(x, y)$ denote the open interval $\{z \in P: x<z<y\}$ and let $[x, y]$ denote the closed interval $\{z \in P: x \leq z \leq y\}$. Half open intervals $(x, y]$ and $[x, y)$ are defined similarly.

If $P$ has a unique minimum element, it is usual to denote it by $\hat{0}$ and refer to it as the bottom element. Similarly, the unique maximum element, if it exists, is denoted $\hat{1}$ and is referred to as the top element. Note that if $P$ has a bottom element $\hat{0}$ or top element $\hat{1}$ then $\Delta(P)$ is contractible since it is a cone. We usually remove the top and bottom elements and study the more interesting topology of the remaining poset. Define the proper part of a poset $P$, for which $|P|>1$, to be

$$
\bar{P}:=P-\{\hat{0}, \hat{1}\} .
$$

In the case that $|P|=1$, it will be convenient to define $\Delta(\bar{P})$ to be the degenerate empty complex $\emptyset$. We will also say $\Delta((x, y))=\emptyset$ and $l((x, y))=-2$ if $x=y$.

For posets with a bottom element $\hat{0}$, the elements that cover $\hat{0}$ are called atoms. For posets with a top element $\hat{1}$, the elements that are covered by $\hat{1}$ are called coatoms.

A poset $P$ is said to be bounded if it has a top element $\hat{1}$ and a bottom element $\hat{0}$. Given a poset $P$, we define the bounded extension

$$
\hat{P}:=P \cup\{\hat{0}, \hat{1}\}
$$

where new elements $\hat{0}$ and $\hat{1}$ are adjoined (even if $P$ already has a bottom or top element).

A poset $P$ is said to be a meet semilattice if every pair of elements $x, y \in P$ has a meet $x \wedge y$, i.e. an element less than or equal to both $x$ and $y$ that is greater than all other such elements. A poset $P$ is said to be a join semilattice if every pair of elements $x, y \in P$ has a join $x \vee y$, i.e. a unique element greater than or equal to both $x$ and $y$ that is less than all other such elements. If $P$ is both a join semilattice and a meet semilattice then $P$ is said to be a lattice. It is a basic fact of lattice theory that any finite meet (join) semilattice with a top (bottom) element is a lattice.

The dual of a poset $P$ is the poset $P^{*}$ on the same underlying set with the order relation reversed. Topologically there is no difference between a poset and its dual since $\Delta(P)$ and $\Delta\left(P^{*}\right)$ are identical simplicial complexes. The direct product $P \times Q$ of two posets $P$ and $Q$ is the poset whose underlying set is the cartesian product $\{(p, q): p \in P, q \in Q\}$ and whose order relation is given by

$$
\left(p_{1}, q_{1}\right) \leq_{P \times Q}\left(p_{2}, q_{2}\right) \text { if } p_{1} \leq_{P} p_{2} \text { and } q_{1} \leq_{Q} q_{2} .
$$




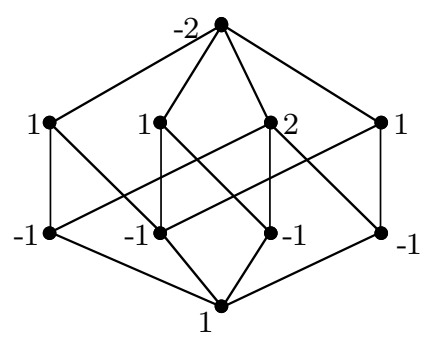

Figure 1.2.1. $\mu(\hat{0}, x)$

Define the join of two simplicial complexes $\Delta$ and $\Gamma$ on disjoint vertex sets to be the simplicial complex given by

$$
\Delta * \Gamma:=\{A \cup B: A \in \Delta, B \in \Gamma\} .
$$

The join (or ordinal sum) $P * Q$ of posets $P$ and $Q$ is the poset whose underlying set is the disjoint union of $P$ and $Q$ and whose order relation is given by $x<y$ if either (i) $x<_{P} y$, (ii) $x<_{Q} y$, or (iii) $x \in P$ and $y \in Q$. Clearly

$$
\Delta(P * Q)=\Delta(P) * \Delta(Q) .
$$

There are topological relationships between the join and product of posets, which are discussed in Section 5.1.

\subsection{The Möbius function}

The story of poset topology begins with the Möbius function $\mu\left(=\mu_{P}\right)$ of a poset $P$ defined recursively on closed intervals of $P$ as follows:

$$
\begin{aligned}
& \mu(x, x)=1, \quad \text { for all } x \in P \\
& \mu(x, y)=-\sum_{x \leq z<y} \mu(x, z), \quad \text { for all } x<y \in P .
\end{aligned}
$$

For a bounded poset $P$, define the Möbius invariant

$$
\mu(P):=\mu_{P}(\hat{0}, \hat{1}) .
$$

In Figure 1.2.1, the values of $\mu(\hat{0}, x)$ are shown for each element $x$ of the poset.

There are various techniques for computing the Möbius function of a poset; see [169]. Perhaps the most basic technique is given by the product formula.

Proposition 1.2.1. Let $P$ and $Q$ be posets. Then for $\left(p_{1}, q_{1}\right) \leq\left(p_{2}, q_{2}\right) \in P \times Q$,

$$
\mu_{P \times Q}\left(\left(p_{1}, q_{1}\right),\left(p_{2}, q_{2}\right)\right)=\mu_{P}\left(p_{1}, p_{2}\right) \mu_{Q}\left(q_{1}, q_{2}\right) .
$$

Exercise 1.2.2. Prove Proposition 1.2.1.

Exercise 1.2.3. Use the product formula to show that the Möbius function for the subset lattice $B_{n}$ is given by

$$
\mu(X, Y)=(-1)^{|Y-X|} \quad \text { for all } X \subseteq Y .
$$


Exercise 1.2.4. For positive integer $n$, the lattice $D_{n}$ of divisors of $n$ is the set of positive divisors of $n$ ordered by $a \leq b$ if $a$ divides $b$. Show that the Möbius function for $D_{n}$ is given by

$$
\mu(d, m)=\mu(m / d)
$$

where $\mu(\cdot)$ is the classical Möbius function of number theory, which is defined on the set of positive integers by

$$
\mu(n)= \begin{cases}(-1)^{k} & \text { if } n \text { is the product of } k \text { distinct primes } \\ 0 & \text { if } n \text { is divisible by a square. }\end{cases}
$$

This example is the reason for the name Möbius function of a poset.

The combinatorial significance of the Möbius function was first demonstrated by Rota in 1964 in his Steele-prize winning paper [147]. The Möbius function of a poset is used in enumerative combinatorics to obtain inversion formulas.

Proposition 1.2.5 (Möbius inversion). Let $P$ be a poset and let $f, g: P \rightarrow \mathbb{C}$. Then

$$
g(y)=\sum_{x \leq y} f(x)
$$

if and only if

$$
f(y)=\sum_{x \leq y} \mu(x, y) g(x) .
$$

Three examples of Möbius inversion are classical Möbius inversion $\left(P=D_{n}\right)$, inclusion-exclusion $\left(P=B_{n}\right)$, and Gaussian inversion $\left(P=B_{n}(q)\right.$, the lattice of subspaces of an $n$-dimensional vector space over the field with $q$ elements); see [169] for details.

Our interest in the Möbius function stems from its connection to the Euler characteristic. The reduced Euler characteristic $\tilde{\chi}(\Delta)$ of a simplicial complex $\Delta$ is defined to be

$$
\tilde{\chi}(\Delta):=\sum_{i=-1}^{\operatorname{dim} \Delta}(-1)^{i} f_{i}(\Delta),
$$

where $f_{i}(\Delta)$ is the number of $i$-faces of $\Delta$.

Proposition 1.2.6 (Philip Hall Theorem). For any poset $P$,

$$
\mu(\hat{P})=\tilde{\chi}(\Delta(P)) .
$$

Exercise 1.2.7. Prove Proposition 1.2.6.

It follows from the Euler-Poincaré formula below that the Euler characteristic is a topological invariant. Hence by Proposition 1.2.6, $\mu_{P}(x, y)$ depends only on the topology of the open interval $(x, y)$ of $P$.

Theorem 1.2.8 (Euler-Poincaré formula). For any simplicial complex $\Delta$,

$$
\tilde{\chi}(\Delta):=\sum_{i=-1}^{\operatorname{dim} \Delta}(-1)^{i} \tilde{\beta}_{i}(\Delta),
$$

where $\tilde{\beta}_{i}(\Delta)$ is the ith reduced Betti number of $\Delta$, i.e., the rank, as an abelian group, of the ith reduced homology of $\Delta$ over $\mathbb{Z}$. 

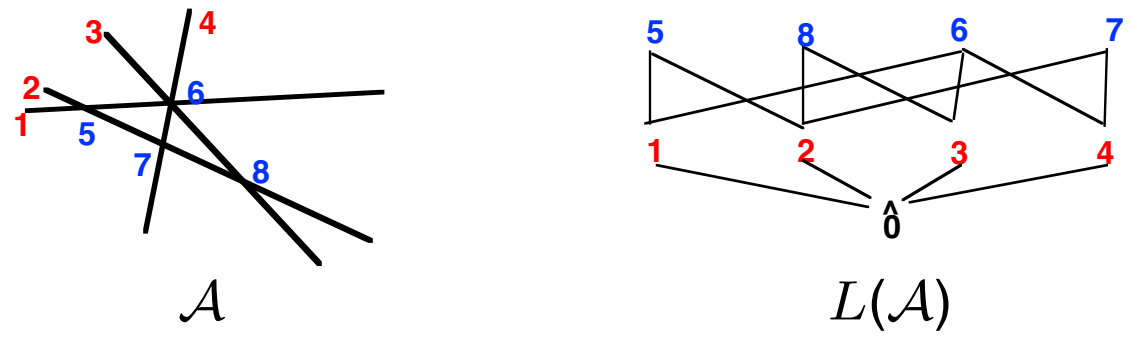

Figure 1.3.1. Intersection semilattice of a hyperplane arrangement

The Möbius function of a poset plays a fundamental role in the theory of hyperplane arrangements and the homology of a poset plays a fundamental role in the theory of subspace arrangements. We discuss the connection with arrangements in the next section.

\subsection{Hyperplane and subspace arrangements}

A hyperplane arrangement $\mathcal{A}$ is a finite collection of (affine) hyperplanes in some vector space $V$. We will consider only real hyperplane arrangements $\left(V=\mathbb{R}^{n}\right)$ and complex hyperplane arrangements $\left(V=\mathbb{C}^{n}\right)$ here.

Real hyperplane arrangements divide $\mathbb{R}^{n}$ into regions. A remarkable formula for the number of regions was given by Zaslavsky [213] in 1975. This formula involves the notion of intersection semilattice of a hyperplane arrangement.

The intersection semilattice $L(\mathcal{A})$ of a hyperplane arrangement $\mathcal{A}$ is defined to be the meet semilattice of nonempty intersections of hyperplanes in $\mathcal{A}$ ordered by reverse inclusion. Note that we include the intersection over the empty set which is the bottom element $\hat{0}$ of $L(\mathcal{A})$. Note also that $L(\mathcal{A})$ has a top element if and only if $\cap \mathcal{A} \neq \emptyset$. Such an arrangement is called a central arrangement. Hence for central arrangements $\mathcal{A}$, the intersection semilattice $L(\mathcal{A})$ is actually a lattice. An example of a hyperplane arrangement in $\mathbb{R}^{2}$ and its intersection semilattice are given in Figure 1.3.1.

Before stating Zaslavsky's formula, we discuss four fundamental examples of real hyperplane arrangements and their intersection lattices, to which we refer throughout these lectures.

Example 1.3.1. The (type A) coordinate hyperplane arrangement and the Boolean algebra $B_{n}$. The coordinate hyperplane arrangement is the central hyperplane arrangement consisting of the coordinate hyperplanes $x_{i}=0$ in $\mathbb{R}^{n}$. It is easy to see that the intersection lattice of this arrangement is isomorphic to the subset lattice $B_{n}$. Indeed, the intersection

$$
\left\{\mathbf{x} \in \mathbb{R}^{n}: x_{i_{1}}=x_{i_{2}}=\cdots=x_{i_{k}}=0\right\},
$$

where $1 \leq i_{1}<i_{2}<\cdots<i_{k} \leq n$, corresponds to the subset $\left\{i_{1}, i_{2}, \ldots, i_{k}\right\}$. This correspondence is an isomorphism from the intersection lattice to $B_{n}$.

Example 1.3.2. The type $B$ coordinate hyperplane arrangement and the face lattice of the $n$-cross-polytope $C_{n}$. The type B coordinate hyperplane arrangement is the 


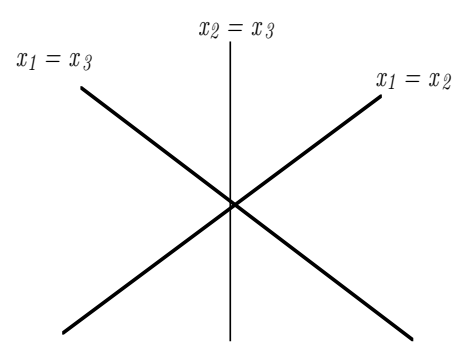

$\mathcal{A}_{2}$

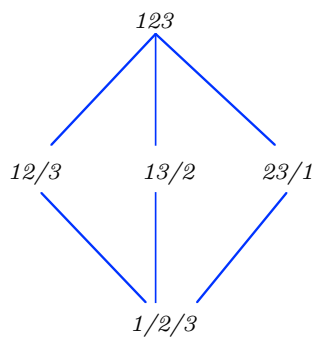

$L\left(\mathcal{A}_{2}\right)=\Pi_{3}$

Figure 1.3.2. Intersection lattice of braid arrangement

affine hyperplane arrangement consisting of the hyperplanes $x_{i}= \pm 1$ in $\mathbb{R}^{n}$. One can see that if we attach a top element $\hat{1}$ to the intersection semilattice of this arrangement we have a lattice that is isomorphic to the lattice of faces of the $n$ cross-polytope, which we denote by $C_{n}$. (This is dual to the lattice of faces of the $n$-cube.) Indeed, the intersection

$$
\left\{\mathbf{x} \in \mathbb{R}^{n}: \epsilon_{1} x_{i_{1}}=\epsilon_{2} x_{i_{2}}=\cdots=\epsilon_{k} x_{i_{k}}=1\right\},
$$

where $1 \leq i_{1}<i_{2}<\cdots<i_{k} \leq n$ and $\epsilon_{i} \in\{-1,1\}$, maps to the $(n-k)$-face

$$
\left\{\mathbf{x} \in[-1,1]^{n}: \epsilon_{1} x_{i_{1}}=\epsilon_{2} x_{i_{2}}=\cdots=\epsilon_{k} x_{i_{k}}=1\right\}
$$

of the $n$-cube. This correspondence is an isomorphism from the intersection lattice to the dual of the face lattice of the cube.

Example 1.3.3. The (type A) braid arrangement and the partition lattice $\Pi_{n}$. For $1 \leq i<j \leq n$, let

$$
H_{i, j}=\left\{\mathbf{x} \in \mathbb{R}^{n}: x_{i}=x_{j}\right\} .
$$

The hyperplane arrangement

$$
\mathcal{A}_{n-1}:=\left\{H_{i, j}: 1 \leq i<j \leq n\right\}
$$

is known as the braid arrangement or the type A Coxeter arrangement. The intersection lattice $L\left(\mathcal{A}_{n-1}\right)$ is isomorphic to $\Pi_{n}$, the lattice of partitions of the set $[n]$ ordered by refinement. Indeed, for each partition $\pi \in \Pi_{n}$, let $\ell_{\pi}$ be the linear subspace of $\mathbb{R}^{n}$ consisting of all points $\left(x_{1}, \ldots, x_{n}\right)$ such that $x_{i}=x_{j}$ whenever $i$ and $j$ are in the same block of $\pi$. The map $\pi \mapsto \ell_{\pi}$ is a poset isomorphism from $\Pi_{n}$ to $L\left(\mathcal{A}_{n-1}\right)$. The braid arrangement $\mathcal{A}_{2}$ intersected with the plane $x_{1}+x_{2}+x_{3}=0$ and the partition lattice $\Pi_{3}$ are shown in Figure 1.3.2.

Example 1.3.4. The type $B$ braid arrangement and the type $B$ partition lattice $\Pi_{n}^{B}$. For $1 \leq i<j \leq n$, let

$$
H_{i, j}^{+}=\left\{\mathbf{x} \in \mathbb{R}^{n}: x_{i}=x_{j}\right\} \quad \text { and } \quad H_{i, j}^{-}=\left\{\mathbf{x} \in \mathbb{R}^{n}: x_{i}=-x_{j}\right\} .
$$

For $i=1, \ldots n$, let

$$
H_{i}=\left\{\mathbf{x} \in \mathbb{R}^{n}: x_{i}=0\right\} .
$$




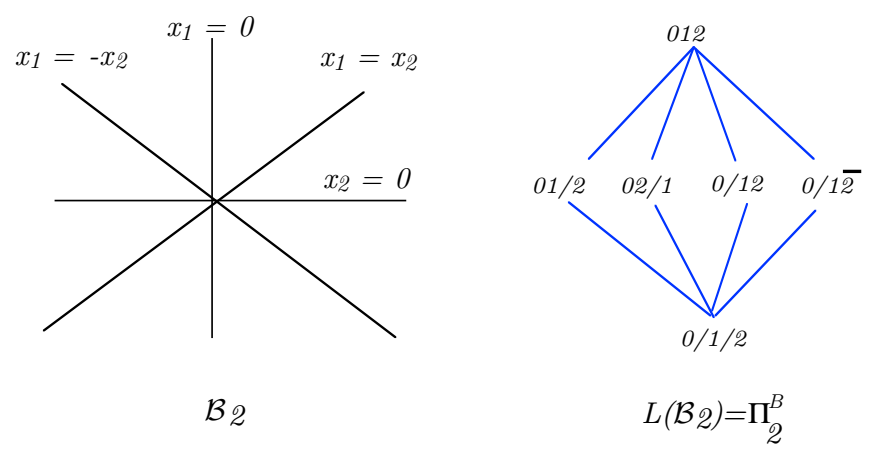

Figure 1.3.3. Intersection lattice of type B braid arrangement

The hyperplane arrangement

$$
\mathcal{B}_{n}:=\left\{H_{i, j}^{+}: 1 \leq i<j \leq n\right\} \cup\left\{H_{i, j}^{-}: 1 \leq i<j \leq n\right\} \cup\left\{H_{i}: 1 \leq i \leq n\right\}
$$

is called the type $B$ braid arrangement or the type $B$ Coxeter arrangement. The intersection lattice $L\left(\mathcal{B}_{n}\right)$ is isomorphic to the type $B$ (or signed) partition lattice. The elements of the type B partition lattice $\Pi_{n}^{B}$ are partitions of $\{0, \ldots, n\}$ for which any of the elements of $[n]$ can have a bar except for the elements of the block

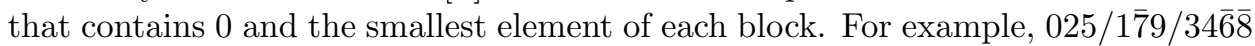
is an element of $\Pi_{9}^{B}$, while $02 \overline{5} / 1 \overline{7} 9 / \overline{3} 4 \overline{6} \overline{8}$ is not because 5 and 3 are not allowed to be barred. The covering relation is given by $\pi_{1} \lessdot \pi_{2}$ if $\pi_{2}$ is obtained from $\pi_{1}$ by merging two blocks $B_{1}$ and $B_{2}$ into a single block $B$ in the following manner: Suppose $\min B_{1}<\min B_{2}$. Then

- if $0 \in B_{1}$, let $B$ be the union of $B_{1}$ and $B_{2}$ with all bars removed,

- if $0 \notin B_{1}$, let $B$ be the union of either

- $B_{1}$ and $B_{2}$ with all bars intact or

- $B_{1}$ and $\bar{B}_{2}$, where $\bar{B}_{2}$ is obtained from $B_{2}$ by barring all unbarred elements and unbarring all barred elements.

For example, the type B partitions that cover $025 / 1 \overline{7} 9 / 34 \overline{6} \overline{8}$ in $\Pi_{9}^{B}$ are

$$
012579 / 34 \overline{6} \overline{8}, \quad 0234568 / 1 \overline{7} 9, \quad 025 / 134 \overline{6} \overline{7} \overline{8} 9, \quad 025 / 1 \overline{3} \overline{4} 6 \overline{7} 89 .
$$

The isomorphism from $\Pi_{n}^{B}$ to $L\left(\mathcal{B}_{n}\right)$ is quite natural. Take a typical type B partition

$$
025 / 1 \overline{7} 9 / 34 \overline{6} \overline{8} \text {. }
$$

It maps to the subspace

$$
\left\{\mathbf{x} \in \mathbb{R}^{9}: x_{2}=x_{5}=0, x_{1}=-x_{7}=x_{9}, x_{3}=x_{4}=-x_{6}=-x_{8}\right\},
$$

in $L\left(\mathcal{B}_{n}\right)$. The type $\mathrm{B}$ braid arrangement $\mathcal{B}_{2}$ and the type $B$ partition lattice $\Pi_{2}^{B}$ are shown in Figure 1.3.3.

Examples 1.3.1 and 1.3.3 are referred to as type A examples, and Examples 1.3.2 and 1.3.4 are referred to as type B examples because of their connection with Coxeter groups. Indeed, associated with every finite Coxeter group (i.e., finite group generated by Euclidean reflections) is a simplicial complex called its Coxeter complex. The order complex of the Boolean algebra $B_{n}$ is the Coxeter complex 
of the symmetric group $\mathfrak{S}_{n}$, which is the type A Coxeter group, and the order complex of the face lattice of the cross-polytope $C_{n}$ is the Coxeter complex of the hyperoctahedral group, which is the type B Coxeter group. (The use of notation is unfortunate here; $B_{n}$ is type $\mathrm{A}$ and $C_{n}$ is type $B$.)

Also associated with every finite Coxeter group is a hyperplane arrangement, called its Coxeter arrangement, which consists of all its reflecting hyperplanes. The group generated by the reflections about hyperplanes in the Coxeter arrangement is the Coxeter group. The braid arrangement is the Coxeter arrangement of the symmetric group (type A Coxeter group) and type B braid arrangement is the Coxeter arrangement of the hyperoctahedral group (type B Coxeter group). Coxeter groups are discussed further in Section 3.3. See the chapters in this volume by Fomin and Reading [71] and Stanley [174] for further discussion of Coxeter arrangements.

The types A and B partition lattices belong to another family of well-studied lattices, namely the Dowling lattices. We will not define Dowling lattices, but we will occasionally refer to them; see [83] for the definition. A broad class of Dowling lattices arise as intersection lattices of complex hyperplane arrangements $\mathcal{A}_{m, n}$ consisting of hyperplanes of the forms $z_{j}=0$, where $j=1, \ldots, n$, and $z_{j}=\omega^{h} z_{i}$, where $\omega$ is the $m$ th primitive root of unity $e^{\frac{2 \pi i}{m}}, 1 \leq i<j \leq n$, and $h \in[m]$. This class includes the types A and B partition lattices.

We now state Zaslavsky's seminal result.

Theorem 1.3.5 (Zaslavsky [213]). Suppose $\mathcal{A}$ is a hyperplane arrangement in $\mathbb{R}^{n}$. Let $r(\mathcal{A})$ be the number of regions into which $\mathcal{A}$ divides $\mathbb{R}^{n}$ and let $b(\mathcal{A})$ be the number of these regions that are bounded. Then

$$
r(\mathcal{A})=\sum_{x \in L(\mathcal{A})}|\mu(\hat{0}, x)|
$$

and

$$
b(\mathcal{A})=|\mu(L(\mathcal{A}) \cup \hat{1})| .
$$

The arrangement of Figure 1.3.1 has a total of 10 regions with 2 of them bounded. One can use the values of the Möbius function given in Figure 1.2.1 to confirm (1.3.1) and (1.3.2) for the arrangement of Figure 1.3.1. Note that if $\mathcal{A}$ is a central arrangement, $L(\mathcal{A}) \cup \hat{1}$ has an artificial top element above the top element of $L(\mathcal{A})$. In other words $L(\mathcal{A}) \cup \hat{1}$ has exactly one coatom. It is easy to see that posets with only one coatom have Möbius invariant 0 . Since central arrangements clearly have no bounded regions, (1.3.2) is trivial for central arrangements.

Exercise 1.3.6. Suppose we have a hyperplane $H$ of $\mathbb{R}^{n}$ which is generic with respect to a central hyperplane arrangement $\mathcal{A}$ in $\mathbb{R}^{n}$. This means that $\operatorname{dim}(H \cap$ $X)=\operatorname{dim}(X)-1$ for all $X \in L(\mathcal{A})$. Let $\mathcal{A}_{H}=\{H \cap K: K \in \mathcal{A}\}$. This is a hyperplane arrangement induced in $H \cong R^{n-1}$. Show that the number of bounded regions of $\mathcal{A}_{H}$ is independent of the choice of generic hyperplane $H$ (see [42]).

The next major development in the combinatorial theory of hyperplane arrangements is a 1980 formula of Orlik and Solomon [129], which can be viewed as a complex analog of (1.3.1). The number of regions in a real hyperplane arrangement $\mathcal{A}$ is equal to the sum of all the Betti numbers of the complement $\mathbb{R}^{n}-\cup \mathcal{A}$. Indeed, since each region is contractible, all the Betti numbers are 0 except for the degree 0 Betti number, which equals the number of regions. Hence (1.3.1) can 
be interpreted as a formula for the sum of the Betti numbers of the complement $\mathbb{R}^{n}-\cup \mathcal{A}$. The analog for complex arrangements is given by following result.

Theorem 1.3.7 (Orlik and Solomon [129]). Let $\mathcal{A}$ be a hyperplane arrangement in $\mathbb{C}^{n}$. The complement $M_{\mathcal{A}}:=\mathbb{C}^{n}-\cup \mathcal{A}$ has torsion-free integral cohomology and has Betti numbers given by,

$$
\beta_{i}\left(M_{\mathcal{A}}\right)=\sum_{\substack{x \in L(\mathcal{A}) \\ \operatorname{dim}^{\mathrm{C}}(x)=n-i}}|\mu(\hat{0}, x)|,
$$

for all $i$.

There is a striking common generalization of the Zaslavsky formula (1.3.1) and the Orlik-Solomon formula, obtained by Goresky and MacPherson in 1988, which involves subspace arrangements. A real subspace arrangement is a finite collection of (affine) subspaces in $\mathbb{R}^{n}$. Real hyperplane arrangements and complex hyperplane arrangements are both examples of real subspace arrangements. Indeed, hyperplanes in $\mathbb{C}^{n}$ can be viewed as codimension 2 subspaces of $\mathbb{R}^{2 n}$. Again the intersection semilattice $L(\mathcal{A})$ is defined to be the semilattice of nonempty intersections of subspaces in the subspace arrangement $\mathcal{A}$.

Theorem 1.3.8 (Goresky and MacPherson $[\mathbf{8 0}]$ ). Let $\mathcal{A}$ be a subspace arrangement in $\mathbb{R}^{n}$. The reduced integral cohomology of the complement $M_{\mathcal{A}}:=\mathbb{R}^{n}-\cup \mathcal{A}$ is given by the group isomorphism

$$
\tilde{H}^{i}\left(M_{\mathcal{A}} ; \mathbb{Z}\right) \cong \bigoplus_{x \in L(\mathcal{A}) \backslash\{\hat{0}\}} \tilde{H}_{n-\operatorname{dim} x-2-i}((\hat{0}, x) ; \mathbb{Z}),
$$

for all $i$.

To see that the Goresky-MacPherson formula reduces to the Zaslavsky formula and to the Orlik-Solomon formula, one needs to understand the homology of the intersection lattice of a central hyperplane arrangement. The intersection lattice belongs to a well-understood class of lattices called geometric lattices. A fundamental result due to Folkman [70] states that the proper part of any geometric lattice $L$ has vanishing reduced homology in every dimension except the top dimension (i.e. dimension equal to $l(L)-2$ ). In fact, the homotopy type is that of a wedge of spheres of top dimension. The intersection lattice of an affine hyperplane arrangement belongs to a more general class of lattices called geometric semilattices, which were introduced and studied by Wachs and Walker [203]. The proper part of a geometric semilattice also has the homotopy type of a wedge of spheres of top dimension. Topology of geometric (semi)lattices is discussed further in Sections 3.2.3 and 4.2 .

Exercise 1.3.9. Use Folkman's result to show that the Goresky-MacPherson formula reduces to both the Zaslavsky formula and the Orlik-Solomon formula.

The intersection lattice of a hyperplane arrangement determines more than the additive group structure of the integral cohomology of the complement. Orlik and Solomon show that it determines the ring structure as well. Ziegler [216] showed that, in general, for subspace arrangements the combinatorial data (intersection lattice and dimension information) does not determine ring structure. However in certain special cases the combinatorial data does determine the cohomology 
algebra, see [69], [212], [58]. In Section 5.4 we discuss some stronger versions of the Goresky-MacPherson formula, namely a homotopy version due to Ziegler and Živaljević [220], and an equivariant version due to Sundaram and Welker [184]. For further reading on hyperplane arrangements, see the chapter by Stanley in this volume [174] and the text by Orlik and Terao [130]. Further information on subspace arrangements can be found in Björner [28] and Ziegler [214].

\subsection{Some connections with graphs, groups and lattices}

In this section we briefly discuss some results and questions in which poset topology plays a role. We start with a old conjecture of Karp in graph complexity theory. An algorithm for deciding whether a graph with $n$ nodes has a certain property checks the entries of the graph's adjacency matrix until a determination can be made. A graph property is said to be evasive if the best algorithm needs to check all $\left(\begin{array}{l}n \\ 2\end{array}\right)$ entries (in the worst case). Here are some examples of evasive graph properties:

- property of being connected

- property of containing a perfect matching

- property of having degree at most $b$ for some fixed $b$.

A monotone graph property is a property of graphs that is isomorphism invariant and closed under addition of edges or closed under removal of edges. The graph properties listed above are clearly monotone graph properties. We say that a graph property is trivial if every graph has the property or every graph lacks the property.

Conjecture 1.4.1 (Karp's Evasiveness Conjecture). Every nontrivial monotone graph property is evasive.

Kahn, Saks, and Sturtevant [106] proved the evasiveness conjecture for $n$ a prime power by using topological techniques and group actions. Since determining whether a graph has a certain property is equivalent to determining whether the graph lacks the property, one can require without loss of generality that a monotone graph property be closed under removal of edges. Given such a monotone graph

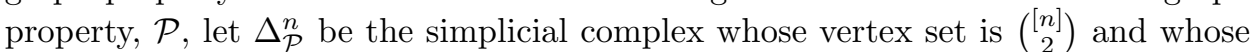
faces are the edge sets of graphs on node set $[n]$ that have the property. Alternatively, $\Delta_{\mathcal{P}}^{n}$ is the simplicial complex whose face poset is the poset of graphs on node set $[n]$ that have property $\mathcal{P}$, ordered by edge set inclusion. Kahn, Saks, and Sturtevant show that

- nonvanishing reduced simplicial homology of $\Delta_{\mathcal{P}}^{n}$ implies $\mathcal{P}$ is evasive

- $\Delta_{\mathcal{P}}^{n}$ has nonvanishing reduced simplicial homology when $n$ is a prime power, using a topological fixed point theorem.

Although this connection between evasiveness and topology doesn't really involve posets directly, we mention it here because posets and simplicial complexes can be viewed as the same object, and as we will see in later in these lectures, the tools of poset topology are useful in the study of the topology of graph complexes. For other significant results on evasiveness and topology of graph complexes, see eg., [55], [211], [73]. This topic is discussed in greater depth in Forman's chapter of this volume [74]. Applications of graph complexes in knot theory and group theory are discussed in Section 5.2. There are also connections between the topology of graph complexes and commutative algebra, which are explored in the work of 
Reiner and Roberts [138] and Dong [61]. A direct application of poset topology in a different complexity theory problem is discussed in Section 3.2.4.

Representability questions in lattice theory deal with whether an arbitrary lattice can be represented as a sublattice, subposet or interval in a given class of lattices. We briefly discuss three examples that have connections to poset topology.

A result of Pudlák and Tuma [134] states that every lattice is isomorphic to a sublattice of some partition lattice $\Pi_{n}$. This implies that every lattice can be represented as the intersection lattice of a subspace arrangement embedded in the braid arrangement. There is another representability result that is much easier to prove; namely that every meet semilattice can be represented as the intersection semilattice of some subspace arrangement, see [214]. From either of these representability results, we see that, in contrast to the situation with hyperplane arrangements, where the topology of the proper part of the intersection semilattice is rather special (a wedge of spheres), any topology is possible for the intersection semilattice of a general subspace arrangement. Indeed, given any simplicial complex $\Delta$, there is a linear subspace arrangement $\mathcal{A}$ such that $\overline{L(\mathcal{A})}$ is homeomorphic to $\Delta$; namely $\mathcal{A}$ is the linear subspace arrangement whose intersection lattice $L(\mathcal{A})$ is isomorphic to the face lattice $L(\Delta)$.

An open representability question is whether every lattice can be represented as an interval in the lattice of subgroups of some group ordered by inclusion. An approach to obtaining a negative answer to this question, proposed by Shareshian [153], is to establish restrictions on the topology of intervals in the subgroup lattice.

Conjecture 1.4.2 (Shareshian [153]). Let $G$ be a finite group. Then every open interval in the lattice of subgroups of $G$ has the homotopy type of a wedge of spheres.

This conjecture was shown to hold for solvable groups by Kratzer and Thévenaz [112] (see Theorem 3.1.13 which strengthens the Kratzer-Thévenaz result). Further discussion of connections between poset topology and group theory can be found in Section 5.2

Our last example deals with the order dimension of a poset $P$, which is defined to be the smallest integer $n$ such that $P$ can be represented as an induced subposet of a product of $n$ chains. Order dimension is an important and extensively studied poset invariant, see [189]. Reiner and Welker give a lower bound on order dimension of a lattice in terms of its homology.

Theorem 1.4.3 (Reiner and Welker [141]). Let $L$ be a lattice and let $d$ be the largest dimension for which the reduced integral simplicial homology of the proper part of $L$ is nonvanishing. Then the order dimension of $L$ is at least $d+2$.

\subsection{Poset homology and cohomology}

By (co)homology of a poset, we usually mean the reduced simplicial (co)homology of its order complex. On rare occasions, we will deal with nonreduced simplicial homology. Although it is presumed that the reader is familiar with simplicial homology and cohomology, we review these concepts for posets in terms of chains of the poset. For each poset $P$ and integer $j$, define the chain space

$$
C_{j}(P ; \mathbf{k}):=\mathbf{k} \text {-module freely generated by } j \text {-chains of } P,
$$

where $\mathbf{k}$ is a field or the ring of integers. 
The boundary map $\partial_{j}: C_{j}(P ; \mathbf{k}) \rightarrow C_{j-1}(P ; \mathbf{k})$ is defined by

$$
\partial_{j}\left(x_{1}<\cdots<x_{j+1}\right)=\sum_{i=1}^{j+1}(-1)^{i}\left(x_{1}<\cdots<\hat{x}_{i}<\cdots<x_{j+1}\right),
$$

where the $\hat{\bullet}$ denotes deletion. We have that $\partial_{j-1} \partial_{j}=0$, which makes $\left(C_{j}(P ; \mathbf{k}), \partial_{j}\right)$ an algebraic complex. Define the cycle space $Z_{j}(P ; \mathbf{k}):=\operatorname{ker} \partial_{j}$ and the boundary space $B_{j}(P ; \mathbf{k}):=\operatorname{im} \partial_{j+1}$. Homology of the poset $P$ in dimension $j$ is defined by

$$
\tilde{H}_{j}(P ; \mathbf{k}):=Z_{j}(P ; \mathbf{k}) / B_{j}(P ; \mathbf{k}) .
$$

The coboundary map $\delta_{j}: C_{j}(P ; \mathbf{k}) \rightarrow C_{j+1}(P ; \mathbf{k})$ is defined by

$$
\left\langle\delta_{j}(\alpha), \beta\right\rangle=\left\langle\alpha, \partial_{j+1}(\beta)\right\rangle
$$

where $\alpha \in C_{j}(P ; \mathbf{k}), \beta \in C_{j+1}(P ; \mathbf{k})$, and $\langle\cdot, \cdot\rangle$ is the bilinear form on $\oplus_{j \geq-1} C_{j}(P ; \mathbf{k})$ for which the chains of $P$ form an orthonormal basis. This is equivalent to saying

$$
\begin{gathered}
\delta_{j}\left(x_{1}<\cdots<x_{j}\right)= \\
\sum_{i=1}^{j+1}(-1)^{i} \sum_{x \in\left(x_{i-1}, x_{i}\right)}\left(x_{1}<\cdots<x_{i-1}<x<x_{i}<\cdots<x_{j}\right),
\end{gathered}
$$

for all chains $x_{1}<\cdots<x_{j}$, where $x_{0}$ is the bottom element of $\hat{P}$ and $x_{j+1}$ is the top element of $\hat{P}$. Define the cocycle space to be $Z^{j}(P ; \mathbf{k}):=\operatorname{ker} \delta_{j}$ and the coboundary space to be $B^{j}(P ; \mathbf{k}):=\operatorname{im} \delta_{j-1}$. Cohomology of the poset $P$ in dimension $j$ is defined to be

$$
\tilde{H}^{j}(P ; \mathbf{k}):=Z^{j}(P ; \mathbf{k}) / B^{j}(P ; \mathbf{k}) .
$$

When $\mathbf{k}$ is a field, $\tilde{H}^{j}(P ; \mathbf{k})$ and $\tilde{H}_{j}(P ; \mathbf{k})$ are isomorphic vector spaces. The $j$ th (reduced) Betti number of $P$ is given by

$$
\tilde{\beta}_{j}(P):=\operatorname{dim} \tilde{H}_{j}(P ; \mathbb{C}),
$$

which is the same as the rank of the free part of $\tilde{H}_{j}(P ; \mathbb{Z})$.

We will work primarily with homology over $\mathbb{C}$ and $\mathbb{Z}$. For $x<y$ in $P$, we write $\tilde{H}_{j}(x, y)$ for the complex homology of the open interval $(x, y)$ of $P$, and $\tilde{\beta}_{j}(x, y)$ for the $j$ th Betti number of the open interval $(x, y)$. When $x=y$, define $\tilde{H}_{j}(x, y)$ to be $\mathbb{C}$ and $\tilde{\beta}_{j}(x, y)$ to be 1 if $j=-2$, and to be 0 for all other $j$.

Many of the posets that arise have the homotopy type of a wedge of spheres. We review a basic fact pertaining to wedges of spheres and a partial converse.

Theorem 1.5.1. Suppose $\Delta$ has the homotopy type of a wedge of spheres of various dimensions, where $r_{i}$ is the number of spheres of dimension $i$. Then for each $i=$ $0,1, \ldots, \operatorname{dim} \Delta$,

$$
\tilde{H}_{i}(\Delta ; \mathbb{Z}) \cong \tilde{H}^{i}(\Delta ; \mathbb{Z}) \cong \mathbb{Z}^{r_{i}} .
$$

Theorem 1.5.2. If $\Delta$ is simply connected and has vanishing reduced integral homology in all dimensions but dimension $n$, where homology is free of rank $r$, then $\Delta$ has the homotopy type of a wedge of $r$ spheres of dimension $n$.

The first tool that we mention for computing homology of posets and simplicial complexes is a very efficient computer software package called "SimplicialHomology", developed by Dumas, Heckenbach, Sauders, and Welker [63]. One can run it interactively or download the source file at the web site: 


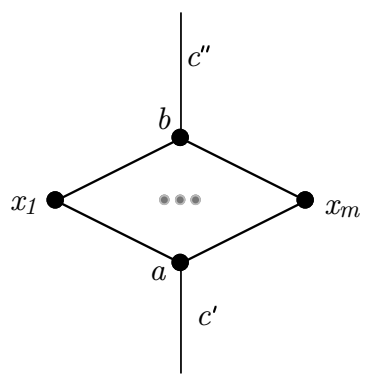

Figure 1.6.1. Top coboundary relations: $\delta\left(c^{\prime} c^{\prime \prime}\right)$

http: //www . cis. udel . edu/ dumas/Homology,

where a manual can also be found. This package has been responsible for many of the more recent conjectures in the field. Its output was also part of the proofs of (at least) three results on integral homology appearing in the literature; see $[154,155,200]$.

\subsection{Top cohomology of the partition lattice}

The top dimensional cohomology of a poset has a particularly simple description. For the sake of simplicity assume $P$ is pure of length $d$. Let $\mathcal{M}(P)$ be the set of maximal chains of $P$ and let $\mathcal{M}^{\prime}(P)$ be the set of chains of length $d-1$. Since $\operatorname{ker} \delta_{d}=C_{d}(P ; \mathbf{k})$, we have the following presentation of top cohomology as a quotient of $C_{d}(P ; \mathbf{k})$ :

$$
\left.\tilde{H}^{d}(P ; \mathbf{k})=\langle\mathcal{M}(P)| \text { coboundary relations }\right\rangle,
$$

where the coboundary relations have the form $\delta_{d-1}(c)$ for $c \in \mathcal{M}^{\prime}(P)$. Each chain $c$ in $\mathcal{M}^{\prime}(P)$ is the concatenation $c^{\prime} c^{\prime \prime}$ of two unrefinable chains $c^{\prime}$ and $c^{\prime \prime}$. If $c^{\prime}$ is not empty, let $a$ be the maximum element of $c_{1}$, and if $c^{\prime}$ is empty, let $a$ be $\hat{0}$ of $\hat{P}$. If $c^{\prime \prime}$ is not empty, let $b$ be the minimum element of $c^{\prime \prime}$, and if $c^{\prime \prime}$ is empty, let $b$ be $\hat{1}$ of $\hat{P}$. Clearly, $[a, b]$ is an interval of length 2 in $\hat{P}$. Let $\left\{x_{1}, \ldots, x_{m}\right\}$ be the set of elements in the open interval $(a, b)$. We have

$$
\delta_{d-1}(c)= \pm\left(c^{\prime} x_{1} c^{\prime \prime}+\cdots+c^{\prime} x_{m} c^{\prime \prime}\right)
$$

Hence the cohomology relations can be associated with the intervals of length 2 in $\hat{P}$. See Figure 1.6.1.

We demonstrate the use of intervals of length 2 by deriving a presentation for the top cohomology of the proper part of the partition lattice $\bar{\Pi}_{n}$. There are two types of length 2 closed intervals in $\Pi_{n}$; see Figure 1.6.2. In Type I intervals, there are 2 pairs of blocks $\{A, B\}$ and $\{C, D\}$ which are separately merged resulting in blocks $A \cup B$ and $C \cup D$. In Type II intervals, there are 3 blocks $A, B, C$, which are merged into one block $A \cup B \cup C$. Type I intervals have 4 elements and type II intervals have 5 elements.

The two types of intervals induce two types of cohomology relations, Type I and Type II cohomology relations on maximal chains. It is convenient to use binary trees on leaf set $[n]$ to describe these relations. A maximal chain of $\bar{\Pi}_{n}$ is 


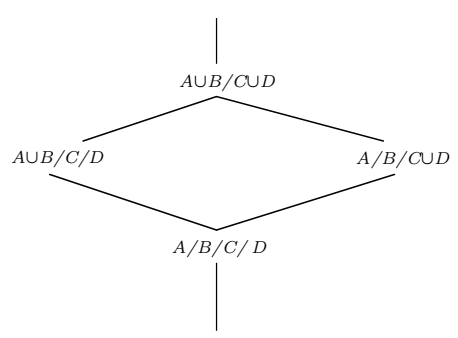

Type I interval

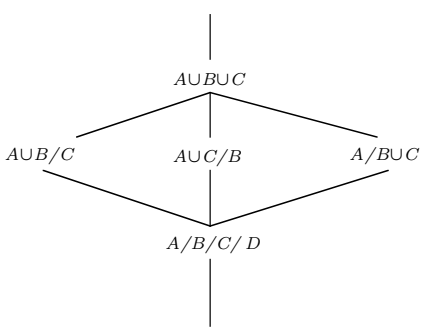

Type II interval

Figure 1.6.2.

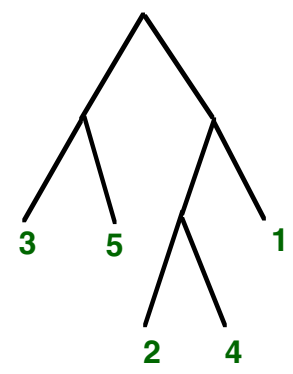

Figure 1.6.3.

just a sequence of merges of pairs of blocks. The binary tree given in Figure 1.6.3 corresponds to the sequence of merges:

(1) merge blocks $\{3\}$ and $\{5\}$

(2) merge blocks $\{2\}$ and $\{4\}$

(3) merge blocks $\{2,4\}$ and $\{1\}$.

This corresponds to the maximal chain

$$
1 / 2 / 35 / 4 \lessdot 1 / 24 / 35 \lessdot 124 / 35
$$

of $\bar{\Pi}_{5}$ The internal nodes of the tree represent the merges, and the leaf sets of the left and right subtrees of the internal nodes are the blocks that are merged. The sequence of merges follows the postorder traversal of the internal nodes, i.e. first traverse the left subtree in postorder, then the right subtree in postorder, then the root.

Given a binary tree $T$ on leaf set $[n]$, let $c(T)$ be the maximal chain of $\Pi_{n}$ obtained by the procedure described above. Although not all maximal chains can be obtained in this way, it can be seen that every maximal chain is equal, modulo the cohomology relations of Type I, to $\pm c(T)$ for some $T$. So the set

$$
\{c(T): T \text { is a binary tree on leaf set }[n]\}
$$

generates top cohomology $\tilde{H}^{n-3}\left(\bar{\Pi}_{n} ; \mathbf{k}\right)$. The Type I cohomology relations induce the following relations

$$
c(\cdots(A \wedge B) \cdots)=(-1)^{|A||B|} c(\cdots(B \wedge A) \cdots),
$$


where $X \wedge Y$ denotes the binary tree whose left subtree is $X$ and whose right subtree is $Y$, and $|X|$ denotes the number of internal nodes of $X$. The Type II cohomology relations induce the following relations

$$
\begin{aligned}
(1.6 .2) c(\cdots(A \wedge(B \wedge C)) \cdots)+ & (-1)^{|C|} c(\cdots((A \wedge B) \wedge C) \cdots) \\
+ & (-1)^{|A| B \mid} c(\cdots(B \wedge(A \wedge C)) \cdots)=0 .
\end{aligned}
$$

Exercise 1.6.1. Show that the Type I cohomology relations yield (1.6.1) and the Type II cohomology relations yield (1.6.2).

The relations (1.6.1) and (1.6.2) resemble the relations satisfied by the bracket operation of a Lie algebra. The Type I relation (1.6.1) corresponds to the anticommuting relation and the Type II relation (1.6.2) corresponds to the Jacobi relation. Indeed there is a well-known connection between the top homology of the partition lattice and the free Lie algebra which involves representations of the symmetric group. In the next lecture, we discuss representation theory.

Theorem 1.6.2 (Stanley [167], Klyachko [108], Joyal [104]). The representation of the symmetric group $\mathfrak{S}_{n}$ on $\tilde{H}_{n-3}\left(\bar{\Pi}_{n} ; \mathbb{C}\right)$ is isomorphic to the representation of $\mathfrak{S}_{n}$ on the multilinear component of the free Lie algebra over $\mathbb{C}$ on $n$ generators tensored with the sign representation.

This result follows from a formula of Stanley for the representation of the symmetric group on homology of the partition lattice (Theorem 4.4.7) and an earlier similar formula of Klyachko for the free Lie algebra. The first purely combinatorial proof was obtained by Barcelo [14]. The presentation of top cohomology discussed above appeared in an alternative combinatorial proof of Wachs [197]. It also appeared in the proof of a superalgebra version of this result obtained by Hanlon and Wachs [91]. A $k$-analog of the Lie superalgebra result was also obtained by Hanlon and Wachs [91]. A type B version (Example 1.3.4) was obtained by Bergeron [16] and a generalization to Dowling lattices was obtained by Gottlieb and Wachs [83]. 



\section{LECTURE 2 \\ Group actions on posets}

In this lecture we give a crash course on the representation theory of the symmetric group and then discuss some representations on homology that are induced by symmetric group actions on posets. For further details on the representation theory of the symmetric group and symmetric functions, we refer the reader to the following excellent standard references $[\mathbf{7 6}, \mathbf{1 2 4}, \mathbf{1 4 9}, \mathbf{1 7 2}]$.

There are various reasons that we are interested in understanding how a group acts on the homology of a poset. One is that this can be a useful tool in computing the homology of the poset. Another is that interesting representations often arise. We limit our discussion to the symmetric group, but point out there are often interesting analogous results for other groups such as the hyperoctahedral group, wreath product groups, and the general linear group.

\subsection{Group representations}

We restrict our discussion to finite groups $G$ and finite dimensional vector spaces over the field $\mathbb{C}$. A finite dimensional vector space $V$ over $\mathbb{C}$ is said to be a representation of $G$ if there is a group homomorphism

$$
\phi: G \rightarrow G L(V) \text {. }
$$

For $g \in G$ and $v \in V$, we write $g v$ instead of $\phi(g)(v)$ and view $V$ as a module over the ring $\mathbb{C} G$ ( $G$-module for short). The dimension of the representation $V$ is defined to be the dimension of $V$ as a vector space.

There are two particular representations of every group that are very important; the trivial representation and the regular representation. The trivial representation, denoted $1_{G}$, is the 1-dimensional representation $V=\mathbb{C}$, where $g z=z$ for all $g \in G$ and $z \in \mathbb{C}$. The (left) regular representation is the $G$-module $\mathbb{C} G$ where $G$ acts on itself by left multiplication, i.e., the action of $g \in G$ on generator $h \in G$ is $g h$.

We say that $V_{1}$ and $V_{2}$ are isomorphic representations of $G$ and write $V_{1} \cong_{G} V_{2}$, if there is a vector space isomorphism $\psi: V_{1} \rightarrow V_{2}$ such that

$$
\psi(g v)=g \psi(v)
$$

for all $g \in G$ and $v \in V_{1}$. In other words, $V_{1}$ and $V_{2}$ are isomorphic representations of $G$ means that they are isomorphic $G$-modules. 
The character of a $G$-module $V$ is a function $\chi^{V}: G \rightarrow \mathbb{C}$ defined by

$$
\chi^{V}(g)=\operatorname{trace}(\phi(g)) \text {. }
$$

One basic fact of representation theory is that the character of a representation completely determines the representation. Another is that $\chi^{V}(g)$ depends only on the conjugacy class of $g$.

A $G$-module $V$ is said to be irreducible if its only submodules are the trivial submodule 0 and $V$ itself. A basic result of representation theory is that the number of irreducible representations of $G$ is the same as the number of conjugacy classes of $G$. Another very important fact is that every $G$-module decomposes into a direct sum of irreducible submodules,

$$
V \cong_{G} V_{1} \oplus \cdots \oplus V_{m}
$$

The decomposition is unique (up to order and up to isomorphism). Hence it makes sense to talk about the multiplicity of an irreducible in a representation. We have the following fundamental fact.

Theorem 2.1.1. The multiplicity of any irreducible representation of $G$ in the regular representation of $G$ is equal to the dimension of the irreducible.

There are two operations on representations that are quite useful. The first is called restriction. For $H$ a subgroup of $G$ and $V$ a representation of $G$, the restriction of $V$ to $H$, denoted $V \downarrow_{H}^{G}$, is the representation of $H$ obtained by restricting $\phi$ to $H$. Thus the restriction has the same underlying vector space with a smaller group action. The other operation, which is called induction, is a bit more complicated. For $H$ a subgroup of $G$ and $V$ a representation of $H$, the induction of $V$ to $G$ is given by

$$
V \uparrow_{H}^{G}:=\mathbb{C} G \otimes_{\mathbb{C} H} V
$$

where the tensor product $A \otimes_{S} B$ denotes the usual tensor product of an $(R, S)$ bimodule $A$ and a left $S$-module $B$ resulting in a left $R$-module. Now the underlying vector space of the induction is larger than $V$.

Exercise 2.1.2. Show

$$
\operatorname{dim} V \uparrow_{H}^{G}=\frac{|G|}{|H|} \operatorname{dim} V .
$$

Although restriction and induction are not inverse operations, they are related by a formula called Frobenius reciprocity. We will state an important special case, which is, in fact, equivalent to Frobenius reciprocity.

Theorem 2.1.3. Let $U$ be an irreducible representation of $H$ and let $V$ be an irreducible representation of $G$, where $H$ is a subgroup of $G$. Then the multiplicity of $U$ in $V \downarrow_{H}^{G}$ is equal to the multiplicity of $V$ in $U \uparrow_{H}^{G}$.

There are two types of tensor products of representations. Given a representation $U$ of $G$ and a representation $V$ of $H$, the (outer) tensor product $U \otimes V$ is a representation of $G \times H$ defined by $(g, h)(u, v)=(g u, h v)$. Given two representations $U$ and $V$ of $G$, the (inner) tensor product, also denoted $U \otimes V$, is the representation of $G$ defined by $g(u, v)=(g u, g v)$.

In these lectures we will describe representations in any of the following ways:

- giving the character

- giving an isomorphic representation 
- giving the multiplicity of each irreducible

- using operations such as restriction, induction and tensor product.

\subsection{Representations of the symmetric group}

In this section, we construct the irreducible representations of the symmetric group $\mathfrak{S}_{n}$, which are called Specht modules and are denoted by $S^{\lambda}$, where $\lambda$ is a partition of $n$. We also discuss skew shaped Specht modules.

Let $\lambda$ be a partition of $n$, i.e., a weakly decreasing sequence of positive integers $\lambda=\left(\lambda_{1} \geq \cdots \geq \lambda_{k}\right)$ whose sum is $n$. We write $\lambda \vdash n$ (or $\left.|\lambda|=n\right)$ and say that the length $l(\lambda)$ is $k$. We will also write $\lambda=1^{m_{1}} 2^{m_{2}} \cdots n^{m_{n}}$, if $\lambda$ has $m_{i}$ parts of size $i$ for each $i$. Each partition $\lambda$ is identified with a Young (or Ferrers) diagram whose $i$ th row has $\lambda_{i}$ cells. For example, the partition $(4,2,2,1) \vdash 9$ is identified with the Young diagram

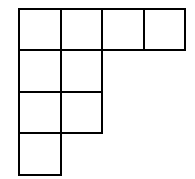

A Young tableau of shape $\lambda \vdash n$ is a filling of the Young diagram corresponding to $\lambda$, with distinct positive integers in $[\mathrm{n}]$. A Young tableau is said to be standard if the entries increase along each row and column. For example, the Young tableau on the left is not standard, while the one on the right is.

\begin{tabular}{|l|l|l|l|}
\hline 8 & 2 & 4 & 1 \\
\hline 7 & 5 & \multicolumn{2}{|l}{} \\
\cline { 1 - 1 } 9 & 3 & \multicolumn{2}{|l}{} \\
\cline { 1 - 1 } 6 & \multicolumn{2}{|l}{} \\
\cline { 1 - 1 }
\end{tabular}

\begin{tabular}{|l|l|l|l|}
\hline 1 & 2 & 4 & 7 \\
\hline 3 & 6 & \multicolumn{2}{|l}{} \\
\cline { 1 - 1 } 5 & 8 & \multicolumn{2}{|c}{} \\
\cline { 1 - 1 } 9 & \multicolumn{2}{|l}{} \\
\cline { 1 - 1 }
\end{tabular}

Let $\mathcal{T}_{\lambda}$ be the set of Young tableaux of shape $\lambda$ and let $M^{\lambda}$ be the complex vector space generated by elements of $\mathcal{T}_{\lambda}$. The symmetric group $\mathfrak{S}_{n}$ acts on $M^{\lambda}$ by permuting entries of the Young tableaux. That is, for transposition $\sigma=(i, j) \in \mathfrak{S}_{n}$, the tableau $\sigma T$ is obtained from $T$ by switching entries $i$ and $j$. For example,

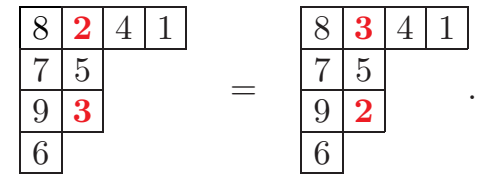

The representation that we have described is clearly the left regular representation of $\mathfrak{S}_{n}$. One can also let $\mathfrak{S}_{n}$ act as the right regular representation on $M^{\lambda}$. That is for transposition $\sigma=(i, j) \in \mathfrak{S}_{n}$ and $T \in \mathcal{T}_{\lambda}$, the tableau $T \sigma$ is obtained from $T$ by switching the contents of the $i$ th and $j$ th cell under some fixed ordering of the cells of $\lambda$.

We will say that two tableaux in $\mathcal{T}_{\lambda}$ are row-equivalent if they have the same sequence of row sets. For example, the tableaux

\begin{tabular}{|c|c|c|c|c|c|c|}
\hline 8 & 2 & $\begin{array}{ll}4 \\
\end{array}$ & 1 & 1 & 2 & \begin{tabular}{l|l}
4 & 8 \\
\end{tabular} \\
\hline 7 & 5 & \multirow{3}{*}{\multicolumn{2}{|c|}{ and }} & 5 & 7 & \\
\hline 9 & 3 & & & 3 & 9 & \\
\hline 6 & & & & 6 & & \\
\hline
\end{tabular}


are row-equivalent. Column-equivalent is defined similarly. For shape $\lambda$, the row stabilizer $R_{\lambda}$ is defined to be the subgroup

$$
R_{\lambda}:=\left\{\sigma \in \mathfrak{S}_{n}: T \sigma \text { and } T \text { are row-equivalent for all } T \in \mathcal{T}_{\lambda}\right\} .
$$

Similarly, the column stabilizer $C_{\lambda}$ is defined to be the subgroup

$$
C_{\lambda}:=\left\{\sigma \in \mathfrak{S}_{n}: T \sigma \text { and } T \text { are column-equivalent for all } T \in \mathcal{T}_{\lambda}\right\} .
$$

We now give two characterizations of the Specht module $S^{\lambda}$; one as a subspace of $M^{\lambda}$ generated by certain signed sums of Young tableaux called polytabloids; and the other as a quotient of $M^{\lambda}$ by certain relations called row relations and Garnir relations. We caution the reader that our notions of polytabloids and Garnir relations are dual to the usual notions given in standard texts such as [149].

We begin with the submodule characterization. For each $T \in \mathcal{T}_{\lambda}$, define the polytabloid of shape $\lambda$,

$$
e_{T}:=\sum_{\alpha \in R_{\lambda}} \sum_{\beta \in C_{\lambda}} \operatorname{sgn}(\beta) T \alpha \beta
$$

For example if

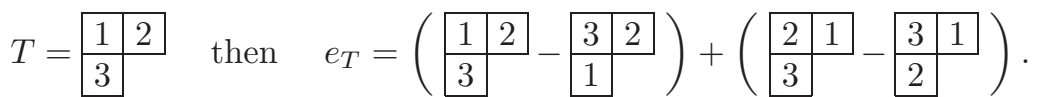

Since the left and right action of $\mathfrak{S}_{n}$ on $\mathcal{T}_{\lambda}$ commute, we have

$$
\pi e_{T}=e_{\pi T},
$$

for all $T \in \mathcal{T}_{\lambda}$ and $\pi \in \mathfrak{S}_{n}$. We can now define the Specht module $S^{\lambda}$ to be the subspace of $M^{\lambda}$ given by

$$
S^{\lambda}:=\left\langle e_{T}: T \in \mathcal{T}_{\lambda}\right\rangle
$$

It follows from (2.2.1) that $S^{\lambda}$ is an $\mathfrak{S}_{n}$-submodule of $M^{\lambda}$ (under the left action).

Theorem 2.2.1. The Specht modules $S^{\lambda}$ for all $\lambda \vdash n$ form a complete set of irreducible $\mathfrak{S}_{n}$-modules.

A polytabloid $e_{T}$ is said to be a standard polytabloid if $T$ is a standard Young tableau. We will see shortly that the standard polytabloids of shape $\lambda$ form a basis for the Specht module $S^{\lambda}$.

Now we give the quotient characterization. The row relations are defined for all $T \in T_{\lambda}$ and $\sigma \in R_{\lambda}$ by

$$
r_{\sigma}(T):=T \sigma-T \text {. }
$$

For all $i, j$ such that $1 \leq j \leq \lambda_{i}$, let $C_{i, j}(\lambda)$ be the set of cells in columns $j$ through $\lambda_{i}$ of row $i$ and in columns 1 through $j$ of row $i+1$. Let $G_{i, j}(\lambda)$ be the subgroup of $\mathfrak{S}_{n}$ consisting of permutations $\sigma$ that fix all entries of the cells that are not in $C_{i, j}(\lambda)$ under the right action of $\sigma$ on tableaux of shape $\lambda$. The Garnir relations are defined for all $i, j$ such that $1 \leq j \leq \lambda_{i}$ and for all $T \in \mathcal{T}_{\lambda}$ by

$$
g_{i, j}(T):=\sum_{\sigma \in G_{i, j}(\lambda)} T \sigma .
$$

For example if

$$
T=\begin{array}{r|r|r|r|}
\hline 7 & \mathbf{1} & \mathbf{5} & \mathbf{1 0} \\
\hline \mathbf{3} & \mathbf{4} & 2 & \\
\cline { 1 - 2 } 9 & 8 & &
\end{array} \quad \text { and } \quad(i, j)=(1,2)
$$


then the entries 1, 5, 10,3, 4 are permuted while the remaining entries are fixed. So

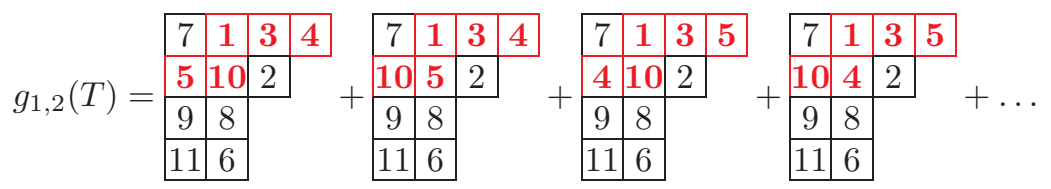

Again, since the left and right action of $\mathfrak{S}_{n}$ on $\mathcal{T}_{\lambda}$ commute, we have

$$
\begin{aligned}
\pi r_{\sigma}(T) & =r_{\sigma}(\pi T) \\
\pi g_{i, j}(T) & =g_{i, j}(\pi T),
\end{aligned}
$$

for all $\pi \in \mathfrak{S}_{n}$. Consequently, the subspace $U^{\lambda}$ of $M^{\lambda}$ generated by the row relations (2.2.2) and the Garnir relations (2.2.3) is an $\mathfrak{S}_{n}$-submodule of $M^{\lambda}$.

Theorem 2.2.2. For all $\lambda \vdash n$,

$$
S^{\lambda} \cong \mathfrak{S}_{n} M^{\lambda} / U^{\lambda}
$$

Now we can view the Specht module $S^{\lambda}$ as the module generated by tableaux of shape $\lambda$ subject to the row and Garnir relations.

Exercise 2.2.3. Prove Theorem 2.2 .2 by first showing that,

(a) $U^{\lambda} \subseteq \operatorname{ker} \psi$, where $\psi: M^{\lambda} \rightarrow S^{\lambda}$ is defined by $\psi(T)=e_{T}$,

(b) the standard polytabloids $e_{T}$ are linearly independent,

(c) the standard tableaux span $M^{\lambda} / U^{\lambda}$.

We have the following consequence of Exercise 2.2.3.

Corollary 2.2.4. The standard polytabloids of shape $\lambda$ form a basis for $S^{\lambda}$. The standard tableaux of shape $\lambda$ form a basis for $M^{\lambda} / U^{\lambda}$. Consequently $\operatorname{dim} S^{\lambda}$ is equal to the number of standard tableaux of shape $\lambda$.

There is a remarkable formula for the number of standard tableaux of a fixed shape $\lambda$.

Theorem 2.2.5 (Frame-Robinson-Thrall hook length formula). For all $\lambda \vdash n$,

$$
\operatorname{dim} S^{\lambda}=\frac{n !}{\prod_{x \in \lambda} h_{x}},
$$

where the product is taken over all cells $x$ in the Young diagram $\lambda$, and $h_{x}$ is the number of cells in the hook formed by $x$, which consists of $x$, the cells that are below $x$ in the same column, and the cells to the right of $x$ in the same row.

One can generalize Specht modules to skew shapes. By removing a smaller skew diagram $\mu$ from the northwest corner of a skew diagram $\lambda$, one gets a skew diagram denoted by $\lambda / \mu$. For example if $\lambda=(4,3,3)$ and $\mu=(2,1)$ then

$$
\lambda / \mu=\square \text {. }
$$

Skew Specht modules $S^{\lambda / \mu}$ are defined analogously to "straight" Specht modules. There is a submodule characterization and a quotient characterization. Theorem 2.2.2 and Corollary 2.2.4 hold in the skew setting. There is a classical combinatorial rule for decomposing Specht modules of skew shape into irreducible straight shape Specht modules called the Littlewood-Richardson rule, which we will not present here. 
Example 2.2.6. Some important classes of skew and straight Specht modules are listed below.

$$
\begin{array}{ll}
\lambda / \mu= & \square \\
\lambda \quad=\square \cdots \square & S^{\lambda / \mu}=\text { regular representation. } \\
\lambda & \square \\
\lambda= & S^{\lambda}=\text { trivial representation }
\end{array}
$$

where the sign representation is the 1-dimensional representation $V=\mathbb{C}$ whose character is $\operatorname{sgn}(\sigma)$, that is $\sigma z=\operatorname{sgn}(\sigma) z$ for all $\sigma \in \mathfrak{S}_{n}$ and $z \in \mathbb{C}$. We denote the sign representation by $\operatorname{sgn}_{n}$ or $S^{\left(1^{n}\right)}$ and we denote the trivial representation by $1_{\mathfrak{S}_{n}}$ or $S^{(n)}$.

Exercise 2.2.7. For skew or straight shape $\lambda / \mu$, let $\chi^{\lambda / \mu}$ denote the character of the representation $S^{\lambda / \mu}$, and for $\sigma \in \mathfrak{S}_{n}$, let $f(\sigma)$ denote the number of fixed points of $\sigma$.

(a) Show $\chi^{(n-1,1)}(\sigma)=f(\sigma)-1$ for all $\sigma \in \mathfrak{S}_{n}$.

(b) Show $\chi^{(n, 1) /(1)}(\sigma)=f(\sigma)$ for all $\sigma \in \mathfrak{S}_{n}$.

(c) Find the character of each of the representations in Example 2.2.6.

A skew hook is a connected skew diagram that does not contain the subdiagram $(2,2)$. Each cell of a skew hook, except for the southwestern most and northeastern most end cells, has exactly two cells adjacent to it. Each of the end cells has only one cell adjacent to it. Let $H$ be a skew hook with $n$ cells. We label the cells of $H$ with numbers 1 through $n$, starting at the southwestern end cell, moving through the adjacent cells, and ending at the northeastern end cell. For example, we have the labeled skew hook

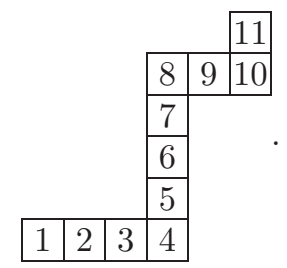

If cell $i+1$ is above cell $i$ in $H$ then we say that the skew hook $H$ has a descent at $i$. Let $\operatorname{des}(H)$ denote the set of descents of $H$. For each subset $S$ of $[n-1]$, there is exactly one skew hook with $n$ cells and descent set $S$. For example, the skew hook

is the only skew hook with 11 cells and descent set $\{4,5,6,7,10\}$.

The Specht modules of skew hook shape are called Foulkes representations. Note that for any skew hook $H$ with $n$ cells, the set of standard tableaux of shape $H$ corresponds bijectively to the set of permutations in $\mathfrak{S}_{n}$ with $\operatorname{descent} \operatorname{set} \operatorname{des}(H)$. (The descent set $\operatorname{des}(\sigma)$ of a permutation $\sigma \in \mathfrak{S}_{n}$ is the set of all $i \in[n-1]$ such 
that $\sigma(i)>\sigma(i+1)$.) Indeed, by listing the entries of cells 1 through $n$, one gets a permutation with descent set $\operatorname{des}(H)$. Hence by Corollary 2.2.4 for skew shapes, the dimension of the Foulkes representation $S^{H}$ is the number of permutations in $\mathfrak{S}_{n}$ with descent set $\operatorname{des}(H)$.

A descent of a standard Young tableau is an entry $i$ that is in a higher row than $i+1$. By applying the Littlewood-Richardson rule mentioned above, one gets the following decomposition of the Foulkes representation into irreducibles,

$$
S^{H}=\bigoplus_{\lambda \vdash n} c_{H, \lambda} S^{\lambda},
$$

where $c_{H, \lambda}$ is the number of standard Young tableaux of shape $\lambda$ and descent set $\operatorname{des}(H)$.

Exercise 2.2.8. Use (2.2.4) to show that the regular representation of $\mathfrak{S}_{n}$ decomposes into Foulkes representations as follows:

$$
\mathbb{C S}_{n} \cong_{\mathfrak{S}_{n}} \bigoplus_{H \in \mathrm{SH}_{n}} S^{H}
$$

where $\mathrm{SH}_{n}$ is the set of skew hooks with $n$ cells.

The induction product of an $\mathfrak{S}_{j}$-module $U$ and an $\mathfrak{S}_{k}$-module $V$ is the $\mathfrak{S}_{j+k^{-}}$ module

$$
U \bullet V:=(U \otimes V) \uparrow_{\mathfrak{S}_{j} \times \mathfrak{S}_{k}}^{\mathfrak{S}_{j+k}} .
$$

(We are viewing $\mathfrak{S}_{j} \times \mathfrak{S}_{k}$ as the subgroup of $\mathfrak{S}_{j+k}$ consisting of permutations that stabilize the sets $\{1,2 \ldots, j\}$ and $\{j+1, j+2, \ldots, j+k\}$.)

Exercise 2.2.9. If a skew shape $D$ consists of two shapes $\lambda$ and $\mu$, where $\lambda$ and $\mu$ have no rows or columns in common, we say that $D$ is the disjoint union of $\lambda$ and $\mu$. Show that $S^{D}=S^{\lambda} \bullet S^{\mu}$ if $D$ is the disjoint union of $\lambda$ and $\mu$. For example

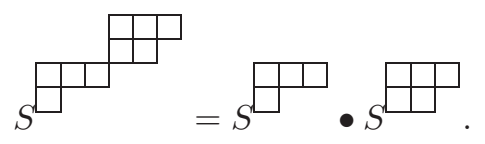

Exercise 2.2.10. Let $\lambda \vdash n$.

(a) Show that

$$
S^{\lambda} \downarrow_{\mathfrak{S}_{n-1}}^{\mathfrak{S}_{n}} \cong_{\mathfrak{S}_{n-1}} \bigoplus_{\mu} S^{\mu}
$$

summed over all Young diagrams $\mu$ obtained from $\lambda$ by removing a cell from the end of one of the rows of $\lambda$. (We are viewing $\mathfrak{S}_{n-1}$ as the subgroup of $\mathfrak{S}_{n}$ consisting of permutations that fix $n$.) For example,

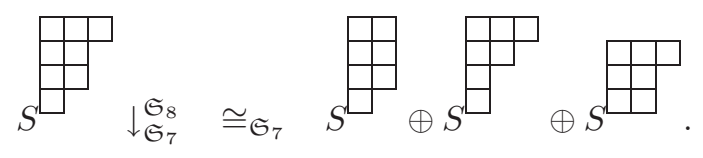

(b) Show that

$$
S^{\lambda} \uparrow_{\mathfrak{S}_{n}}^{\mathfrak{S}_{n+1}}=S^{\lambda} \bullet S^{(1)} \cong_{\mathfrak{S} n+1} \bigoplus_{\mu} S^{\mu}
$$


summed over all Young diagrams $\mu$ obtained from $\lambda$ by adding a cell to the end of one of the rows of $\lambda$. For example,

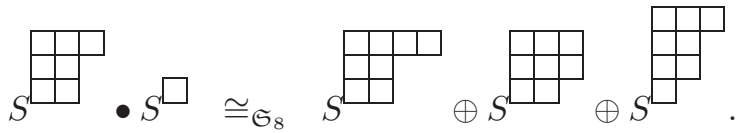

There is an important generalization of Exercise 2.2.10 (b) known as Pieri's rule.

Theorem 2.2.11 (Pieri's rule). Let $m, n \in \mathbb{Z}^{+}$. If $\lambda \vdash n$ then

$$
S^{\lambda} \bullet S^{(m)} \cong_{\mathfrak{S}_{m+n}} \bigoplus_{\mu} S^{\mu},
$$

summed over all partitions $\mu$ of $m+n$ such that $\mu$ contains $\lambda$ and the skew shape $\mu / \lambda$ has at most one cell in each column. Similarly

$$
S^{\lambda} \bullet S^{\left(1^{m}\right)} \cong_{\mathfrak{S}_{m+n}} \bigoplus_{\mu} S^{\mu},
$$

summed over all partitions $\mu$ of $m+n$ such that $\mu$ contains $\lambda$ and the skew shape $\mu / \lambda$ has at most one cell in each row.

The conjugate of a partition $\lambda$ is the partition $\lambda^{\prime}$ whose Young diagram is the transpose of that of $\lambda$.

Theorem 2.2.12. For all partitions $\lambda \vdash n$,

$$
S^{\lambda} \otimes \operatorname{sgn}_{n} \cong \mathfrak{S}_{n} S^{\lambda^{\prime}},
$$

where the tensor product is an inner tensor product. This also holds for skew diagrams.

Exercise 2.2.13. Let $V$ be a representation of $\mathfrak{S}_{n}$. Show that

$$
\left(V \otimes \operatorname{sgn}_{n}\right) \uparrow \mathfrak{S}_{\mathfrak{S}_{n}}^{\mathfrak{S}_{n+1}} \cong_{\mathfrak{S}_{n+1}} V \uparrow_{\mathfrak{S}_{n}}^{\mathfrak{S}_{n+1}} \otimes \operatorname{sgn}_{n+1},
$$

and

$$
\left(V \otimes \operatorname{sgn}_{n}\right) \downarrow_{\mathfrak{S}_{n-1}}^{\mathfrak{S}_{n}} \cong_{\mathfrak{S}_{n-1}} \quad V \downarrow_{\mathfrak{S}_{n-1}}^{\mathfrak{S}_{n}} \otimes \operatorname{sgn}_{n-1} .
$$

\subsection{Group actions on poset (co)homology}

Let $G$ be a finite group. A $G$-simplicial complex is a simplicial complex together with an action of $G$ on its vertices that takes faces to faces. A $G$-poset is a poset together with a $G$-action on its elements that preserves the partial order; i.e., $x<$ $y \Rightarrow g x<g y$. So if $P$ is a $G$-poset then its order complex $\Delta(P)$ is a $G$-simplicial complex and if $\Delta$ is a $G$-simplicial complex then its face poset $P(\Delta)$ is a $G$-poset.

A $G$-space is a topological space on which $G$ acts as a group of homeomorphisms. If $\Delta$ is a $G$-simplicial complex then the geometric realization $\|\Delta\|$ is a $G$-space under the natural induced action of $G$.

Example 2.3.1. The subset lattice $B_{n}$ is an $\mathfrak{S}_{n}$-poset. The action of a permutation $\sigma \in \mathfrak{S}_{n}$ on a subset $\left\{a_{1}, \ldots, a_{k}\right\}$ is given by

$$
\sigma\left\{a_{1}, \ldots, a_{k}\right\}=\left\{\sigma\left(a_{1}\right), \ldots, \sigma\left(a_{k}\right)\right\} .
$$


Example 2.3.2. The partition lattice $\Pi_{n}$ is an $\mathfrak{S}_{n}$-poset. The action of a permutation $\sigma \in \mathfrak{S}_{n}$ on a partition $\left\{B_{1}, \ldots, B_{k}\right\}$ is given by

$$
\sigma\left\{B_{1}, \ldots, B_{k}\right\}=\left\{\sigma B_{1}, \ldots, \sigma B_{k}\right\},
$$

where $\sigma B_{i}$ is defined in (2.3.1). The symmetric group is isomorphic to the group generated by reflections about hyperplanes in the braid arrangement. The action described here is simply the action of the reflection group on intersections of hyperplanes in the braid arrangement.

Example 2.3.3. The face lattice $C_{n}$ of the $n$-cross-polytope (or the face lattice of the $n$-cube) is an $\mathfrak{S}_{n}\left[\mathbb{Z}_{2}\right]$-poset. The wreath product group $\mathfrak{S}_{n}\left[\mathbb{Z}_{2}\right]$ is also known as the hyperoctahedral group or the type B Coxeter group (see Section 2.4 for the definition of wreath product). It is the group generated by reflections about the hyperplanes in the type B braid arrangement.

By viewing the $n$-cross-polytope as the convex hull of the points $\pm e_{i}, i=$ $1, \ldots, n$, one obtains the action of $\mathfrak{S}_{n}\left[\mathbb{Z}_{2}\right]$ on $C_{n}$. We describe this action in combinatorial terms. The $(k-1)$-dimensional faces of the cross-polytope are convex hulls of certain $k$ element subsets of $\left\{ \pm e_{i}: i \in[n]\right\}$. These are the ones that don't contain both $e_{i}$ and $-e_{i}$ for any $i$. Thus the $(k-1)$-faces can be identified with $k$-subsets $T$ of $[n] \cup\{\bar{i}: i \in[n]\}$ such that $\{i, \bar{i}\} \not \subseteq T$ for all $i$. By ordering these sets by containment, one gets the face poset of the $n$-cross-polytope. For example, the 4 -subset $\{3, \overline{5}, 6, \overline{8}\}$ is identified with the convex hull of the points $e_{3},-e_{5}, e_{6},-e_{8}$. The elements of $\mathfrak{S}_{n}\left[\mathbb{Z}_{2}\right]$ are identified with permutations $\sigma$ of $[n] \cup\{\bar{i}: i \in[n]\}$ for which $\overline{\sigma(i)}=\sigma(\bar{i})$ for all $i$ (where $\overline{\bar{a}}=a$ ). Then the action of a permutation $\sigma \in \mathfrak{S}_{n}\left[\mathbb{Z}_{2}\right]$ on a $k$-subset $\left\{a_{1}, \ldots, a_{k}\right\}$ of $[n] \cup\{\bar{i}: i \in[n]\}$ is given by (2.3.1).

Example 2.3.4. The type B partition lattice $\Pi_{n}^{B}$ is also an $\mathfrak{S}_{n}\left[\mathbb{Z}_{2}\right]$-poset. Recall from Example 1.3.4 that the type B partition lattice $\Pi_{n}^{B}$ is the intersection lattice of the type $B$ braid arrangement. Since this arrangement is invariant under reflection about any hyperplane in the arrangement, elements of the reflection group $\mathfrak{S}_{n}\left[\mathbb{Z}_{2}\right]$ map intersections of hyperplanes to intersections of hyperplanes. This gives the action of $\mathfrak{S}_{n}\left[\mathbb{Z}_{2}\right]$ on $\Pi_{n}^{B}$. There is a combinatorial description of the action analogous to $(2.3 .2)$ (see e.g. $[\mathbf{8 3}])$.

Example 2.3.5. The lattice of subspaces of an $n$-dimensional vector space over a finite field $F$ is a $G L_{n}(F)$-poset, where the general linear group $G L_{n}(F)$ acts in the obvious way.

Example 2.3.6. The lattice of subgroups of a finite group $G$ ordered by inclusion is a $G$-poset, where $G$ acts by conjugation.

Example 2.3.7. The semilattice of $p$-subgroups of a group $G$ ordered by inclusion is a $G$-poset, where $G$ acts by conjugation.

Let $P$ be a $G$-poset. Since $g \in G$ takes $j$-chains to $j$-chains, $g$ acts as a linear map on $C_{j}(P ; \mathbb{C})$. It is easy to see that

$$
g \partial(c)=\partial(g c) \text { and } g \delta(c)=\delta(g c) .
$$

Hence $g$ acts as a linear map on $\tilde{H}_{j}(P ; \mathbb{C})$ and on $\tilde{H}^{j}(P ; \mathbb{C})$. This means that whenever $P$ is a $G$-poset, $\tilde{H}_{j}(P ; \mathbb{C})$ and $\tilde{H}^{j}(P ; \mathbb{C})$ are $G$-modules. The bilinear form $(1.5 .1)$, induces a pairing between $\tilde{H}_{j}(P ; \mathbb{C})$ and $\tilde{H}^{j}(P ; \mathbb{C})$, which allows one 
to view them as dual $G$-modules. For $G=\mathfrak{S}_{n}$ or $G=\mathfrak{S}_{n}\left[\mathbb{Z}_{2}\right]$,

$$
\tilde{H}_{j}(P, \mathbb{C}) \cong_{G} \tilde{H}^{j}(P, \mathbb{C})
$$

since dual $\mathfrak{S}_{n}$-modules (resp., dual $\mathfrak{S}_{n}\left[\mathbb{Z}_{2}\right]$-modules) are isomorphic.

Given a $G$-simplicial complex $\Delta$, the natural homeomorphism from $\Delta$ to its barycentric subdivision $\Delta(P(\Delta))$ commutes with the $G$-action. Consequently, for all $j \in \mathbb{Z}$

$$
\tilde{H}_{j}(\Delta ; \mathbb{C}) \cong_{G} \tilde{H}_{j}(P(\Delta) ; \mathbb{C})
$$

and

$$
\tilde{H}^{j}(\Delta ; \mathbb{C}) \cong_{G} \tilde{H}^{j}(P(\Delta) ; \mathbb{C}) .
$$

Exercise 2.3.8. The maximal chains of $\bar{B}_{n}$ correspond bijectively to tableaux of shape $1^{n}$ via the map

$$
\begin{array}{c|c}
\hline t_{1} \\
\hline t_{2} \\
\hline \vdots \\
\hline t_{n} \\
\hline
\end{array} \mapsto \quad\left(\left\{t_{1}\right\} \subset\left\{t_{1}, t_{2}\right\} \subset \cdots \subset\left\{t_{1}, \ldots, t_{n-1}\right\}\right) .
$$

(a) Show that the Garnir relations map to the coboundary relations. Consequently, the representation of $\mathfrak{S}_{n}$ on the top cohomology $\tilde{H}^{n-2}\left(\bar{B}_{n} ; \mathbb{C}\right)$ is the sign representation.

(b) Show that the polytabloids map to cycles in top homology.

There is an equivariant version of the Euler-Poincaré formula (Theorem 1.2.8), known as the Hopf-trace formula. It is convenient to state this formula in terms of virtual representations, which we define first. The representation group $\mathcal{G}(G)$ of a group $G$ is the free abelian group on the set of all isomorphism classes $[V]$ of $G$-modules $V$ modulo the subgroup generated by all $[V \oplus W]-[V]-[W]$. Elements of the representation group are called virtual representations. If $V$ is an actual representation, we denote the virtual representation $[V]$ by $V$. Note that two virtual representations $A-B$ and $C-D$, where $A, B, C, D$ are actual representations of $G$, are equal in the representation group if and only if $A \oplus D \cong_{G} B \oplus C$ in the usual sense. We will write $A-B \cong_{G} C-D$.

Theorem 2.3.9 (Hopf trace formula). For any G-simplicial complex $\Delta$,

$$
\bigoplus_{i=-1}^{\operatorname{dim} \Delta}(-1)^{i} C_{i}(\Delta ; \mathbb{C}) \cong_{G} \bigoplus_{i=-1}^{\operatorname{dim} \Delta}(-1)^{i} \tilde{H}_{i}(\Delta ; \mathbb{C})
$$

We will usually suppress the $\mathbb{C}$ from our notation $\tilde{H}_{i}(P ; \mathbb{C})\left(\right.$ resp., $\tilde{H}^{i}(P ; \mathbb{C})$ ) and write $\tilde{H}_{i}(P)\left(\right.$ resp., $\left.\tilde{H}^{i}(P)\right)$ instead when viewing (co)homology as a $G$-module.

\subsection{Symmetric functions, plethysm, and wreath product modules}

Symmetric functions provide a convenient way of describing and computing representations of the symmetric group. In this section we give the basics of symmetric function theory. Then we demonstrate its use in computing homology of an interesting example known as the matching complex. 


\subsubsection{Symmetric functions}

Let $x=\left(x_{1}, x_{2}, \ldots\right)$ be an infinite sequence of indeterminates. A homogeneous symmetric function of degree $n$ is a formal power series $f(x) \in \mathbb{Q}[[x]]$ in which each term has degree $n$ and $f\left(x_{\sigma(1)}, x_{\sigma(2)}, \ldots\right)=f\left(x_{1}, x_{2}, \ldots\right)$ for all permutations $\sigma$ of $\mathbb{Z}^{+}$.

Let $\Lambda^{n}$ denote the set of homogeneous symmetric functions of degree $n$ and let $\Lambda=\bigoplus_{n>0} \Lambda^{n}$. Then $\Lambda$ is a graded $\mathbb{Q}$-algebra, since $\alpha f(x)+\beta g(x) \in \Lambda^{n}$ if $f(x), g(x) \in \bar{\Lambda}^{n}$ and $\alpha, \beta \in \mathbb{Q}$; and $f(x) g(x) \in \Lambda_{m+n}$ if $f(x) \in \Lambda^{m}$ and $g(x) \in \Lambda^{n}$.

There are several important bases for the vector space $\Lambda^{n}$. We mention just two of them here; the basis of power sum symmetric functions and the basis of Schur functions. These bases are indexed by partitions of $n$. For $n \geq 1$, let

$$
p_{n}=\sum_{i \geq 1} x_{i}^{n}
$$

and let $p_{0}=1$. The power sum symmetric function indexed by $\lambda=\left(\lambda_{1} \geq \lambda_{2} \geq\right.$ $\left.\cdots \geq \lambda_{k}\right)$ is defined by

$$
p_{\lambda}:=p_{\lambda_{1}} p_{\lambda_{2}} \ldots p_{\lambda_{k}} .
$$

Given a Young diagram $\lambda$, a semistandard tableau of shape $\lambda$ is a filling of $\lambda$ with positive integers so that the rows weakly increase and the columns strictly increase. Let $S S_{\lambda}$ be the set of semistandard tableaux of shape $\lambda$. Given a semistandard tableau $T$, define $w(T):=x_{1}^{m_{1}} x_{2}^{m_{2}} \ldots$, where for each $i, m_{i}$ is the number of times $i$ appears in $T$. Now define the Schur function indexed by $\lambda$ to be

$$
s_{\lambda}:=\sum_{T \in S S_{\lambda}} w(T) .
$$

Skew shaped Schur functions $s_{D}$ are defined analogously for all skew diagrams $D$.

While it is obvious that the power sum symmetric functions are symmetric functions, it is not obvious that the Schur functions defined this way are.

Theorem 2.4.1. The sets $\left\{p_{\lambda}: \lambda \vdash n\right\}$ and $\left\{s_{\lambda}: \lambda \vdash n\right\}$ form bases for $\Lambda^{n}$. Moreover, $\left\{s_{\lambda}: \lambda \vdash n\right\}$ is an integral basis, i.e., a basis for the $\mathbb{Z}$-module $\Lambda_{\mathbb{Z}}^{n}$ of homogeneous symmetric functions of degree $n$ with integer coefficients.

The Schur function $s_{(n)}$ is known as the complete homogeneous symmetric function of degree $n$ and is denoted by $h_{n}$. The Schur function $s_{\left(1^{n}\right)}$ is known as the elementary symmetric function of degree $n$ and is denoted by $e_{n}$. There is an important involution $\omega: \Lambda_{n} \rightarrow \Lambda_{n}$ defined by

$$
\omega\left(s_{\lambda}\right)=s_{\lambda^{\prime}},
$$

where $\lambda^{\prime}$ is the conjugate of $\lambda$. Clearly

$$
\omega\left(h_{n}\right)=e_{n} .
$$

For the power sum symmetric functions we have

$$
\omega\left(p_{\lambda}\right)=(-1)^{|\lambda|-l(\lambda)} p_{\lambda}
$$

Exercise 2.4.2. Prove

$$
\sum_{n \geq 0} h_{n}=\prod_{i \geq 1}\left(1-x_{i}\right)^{-1}
$$


and

where $h_{0}=e_{0}=1$.

$$
\sum_{n \geq 0} e_{n}=\prod_{i \geq 1}\left(1+x_{i}\right)
$$

The Frobenius characteristic $\operatorname{ch}(V)$ of a representation $V$ of $\mathfrak{S}_{n}$ is the symmetric function given by

$$
\operatorname{ch}(V):=\sum_{\mu \vdash n} \frac{1}{z_{\mu}} \chi^{V}(\mu) p_{\mu},
$$

where $z_{\mu}:=1^{m_{1}} m_{1} ! 2^{m_{2}} m_{2} ! \ldots n^{m_{n}} m_{n}$ ! for $\mu=1^{m_{1}} 2^{m_{2}} \ldots n^{m_{n}}$ and $\chi^{V}(\mu)$ is the character $\chi^{V}(\sigma)$ for $\sigma \in \mathfrak{S}_{n}$ of conjugacy type $\mu$. Some basic facts on Frobenius characteristic are compiled in the next result.

\section{Theorem 2.4.3.}

(a) For all (skew or straight) shapes $\lambda$,

$$
\operatorname{ch}\left(S^{\lambda}\right)=s_{\lambda}
$$

(b) For all representations $V$ of $\mathfrak{S}_{n}$,

$$
\omega(\operatorname{ch} V)=\operatorname{ch}\left(V \otimes \operatorname{sgn}_{n}\right) .
$$

(c) For all representations $U, V$ of $\mathfrak{S}_{n}$,

$$
\operatorname{ch}(U \oplus V)=\operatorname{ch}(U)+\operatorname{ch}(V)
$$

(d) For all representations $U$ of $\mathfrak{S}_{m}$ and $V$ of $\mathfrak{S}_{n}$,

$$
\operatorname{ch}(U \bullet V)=\operatorname{ch}(U) \operatorname{ch}(V) .
$$

The direct sum $\bigoplus_{n>0} \mathcal{G}\left(\mathfrak{S}_{n}\right)$ of representation groups is a ring under the induction product. It follows from Theorem 2.4.3 that the Frobenius characteristic map is an isomorphism from the ring $\bigoplus_{n \geq 0} \mathcal{G}\left(\mathfrak{S}_{n}\right)$ to the ring of symmetric functions over $\mathbb{Z}$.

Definition 2.4.4. Let $f \in \Lambda$ and let $g$ be a formal power series with positive integer coefficients. Choose any ordering of the monomials of $g$, where a monomial appears in the ordering $m_{i}$ times if its coefficient is $m_{i}$. For example, if $g=$ $3 y_{1} y_{2}^{2}+2 y_{2} y_{3}+\ldots$ then the monomials can be arranged as

$$
\left(y_{1} y_{2}^{2}, y_{1} y_{2}^{2}, y_{1} y_{2}^{2}, y_{2} y_{3}, y_{2} y_{3}, \ldots\right) \text {. }
$$

Pad the sequence of monomials with zero's if $g$ has a finite number of terms. Define the plethysm of $f$ and $g$, denoted $f[g]$, to be the formal power series obtained from $f$ by replacing the indeterminate $x_{i}$ with the $i$ th monomial of $g$ for each $i$. Since $f$ is a symmetric function, the chosen order of the monomials doesn't matter. For example, if $f=\sum_{n \geq 0} e_{n}=\prod_{i \geq 1}\left(1+x_{i}\right)$ and $g$ is as above then

$$
f[g]=\left(1+y_{1} y_{2}^{2}\right)\left(1+y_{1} y_{2}^{2}\right)\left(1+y_{1} y_{2}^{2}\right)\left(1+y_{2} y_{3}\right)\left(1+y_{2} y_{3}\right) \cdots .
$$

The following proposition is immediate.

Proposition 2.4.5. Suppose $f, g \in \Lambda$ and $h$ is a formal power series with positive integer coefficients. Then

- If $f$ has positive integer coefficients then $f\left[p_{n}\right]=p_{n}[f]$ is obtained by replacing each indeterminate $x_{i}$ of $f$ by $x_{i}^{n}$. 
- $(a f+b g)[h]=a f[h]+b g[h]$, where $a, b \in \mathbb{Q}$

- $f g[h]=f[h] g[h]$.

Note that if $g \in \Lambda$ has positive integer coefficients then $f[g] \in \Lambda$. One can extend the definition of plethysm to all $g \in \Lambda$, by using Proposition 2.4.5 and the fact that the power sum symmetric functions form a basis for $\Lambda$. Hence plethysm is a binary operation on $\Lambda$, which is clearly associative, but not commutative. Note that the plethystic identity is $p_{1}=h_{1}$. We say that $f, g \in \Lambda$ are plethystic inverses of each other, and write $g=f^{[-1]}$, if $f[g]=g[f]=h_{1}$.

\subsubsection{Composition product and wreath product}

Our purpose for introducing plethysm in these lectures is that plethysm encodes a product operation on symmetric group representations called composition product, which is described below. (This description is based on an exposition given in [178].)

Let $G$ be a finite group. The wreath product of $\mathfrak{S}_{m}$ and $G$, denoted by $\mathfrak{S}_{m}[G]$, is defined to be the set of $(m+1)$-tuples $\left(g_{1}, g_{2}, \ldots, g_{m} ; \tau\right)$ such that $g_{i} \in G$ and $\tau \in \mathfrak{S}_{m}$ with multiplication given by

$$
\left(g_{1}, \ldots, g_{m} ; \tau\right)\left(h_{1}, \ldots, h_{m} ; \gamma\right)=\left(g_{1} h_{\tau^{-1}(1)}, \ldots, g_{m} h_{\tau^{-1}(m)} ; \tau \gamma\right) .
$$

The following proposition is immediate.

Proposition 2.4.6. The map $\left(\alpha_{1}, \alpha_{2}, \ldots, \alpha_{m} ; \tau\right) \mapsto \tau$ is a homomorphism from $\mathfrak{S}_{m}\left[\mathfrak{S}_{n}\right]$ onto $\mathfrak{S}_{m}$.

Definition 2.4.7. Let $V$ be an $\mathfrak{S}_{m}$-module and $W$ be a $G$-module. Then the wreath product of $V$ with $W$, denoted $V[W]$, is the inner tensor product of two $\mathfrak{S}_{m}[G]$-modules:

$$
V[W]=\widetilde{W^{\otimes m}} \otimes \hat{V}
$$

where $\widetilde{W^{\otimes m}}$ is the vector space $W^{\otimes m}$ with $\mathfrak{S}_{m}[G]$ action given by

$$
\left(\alpha_{1}, \ldots, \alpha_{m} ; \tau\right)\left(w_{1} \otimes \cdots \otimes w_{m}\right)=\alpha_{1} w_{\tau^{-1}(1)} \otimes \cdots \otimes \alpha_{m} w_{\tau^{-1}(m)}
$$

and $\hat{V}$ is the pullback of the representation of $\mathfrak{S}_{m}$ on $V$ to $\mathfrak{S}_{m}[G]$ through the homomorphism given in Proposition 2.4.6. That is, $\hat{V}$ is the representation of $\mathfrak{S}_{m}[G]$ on $V$ defined by

$$
\left(\alpha_{1}, \ldots, \alpha_{m} ; \tau\right) v=\tau v .
$$

Given a finite set $A=\left\{a_{1}<a_{2}<\cdots<a_{n}\right\}$ of positive integers, let $\mathfrak{S}_{A}$ be the set of permutations of the set $A$. We shall view a permutation in $\mathfrak{S}_{A}$ as a word whose letters come from $A$. For $\sigma \in S_{n}$, let $\sigma^{A}$ denote the word $a_{\sigma(1)} a_{\sigma(2)} \cdots a_{\sigma(n)}$. We shall view an element of the Young subgroup $\mathfrak{S}_{k} \times \mathfrak{S}_{n-k}$ of $\mathfrak{S}_{n}$ as the concatenation $\alpha \star \beta$ of words $\alpha \in \mathfrak{S}_{k}$ and $\beta \in \mathfrak{S}_{\{k+1, \ldots, n\}}$. The wreath product $\mathfrak{S}_{m}\left[\mathfrak{S}_{n}\right]$ is isomorphic to the normalizer of the Young subgroup $\underbrace{\mathfrak{S}_{n} \times \cdots \times \mathfrak{S}_{n}}_{m \text { times }}$ in $\mathfrak{S}_{m n}$. The isomorphism is given by

$$
\left(\alpha_{1}, \ldots, \alpha_{m} ; \tau\right) \mapsto \alpha_{\tau(1)}^{A_{\tau(1)}} \star \cdots \star \alpha_{\tau(m)}^{A_{\tau(m)}},
$$

where $A_{i}=[i n] \backslash[(i-1) n]$. 
Define the composition product of an $\mathfrak{S}_{m}$-module $V$ and an $\mathfrak{S}_{n}$-module $W$ by

$$
V \circ W:=V[W] \uparrow \underset{\mathfrak{S}_{m}\left[\mathfrak{S}_{n}\right]}{\mathfrak{S}_{m n}}
$$

The following result relates plethysm to composition product.

Theorem 2.4.8. Let $V$ be an $\mathfrak{S}_{m}$-module and $W$ be an $\mathfrak{S}_{n}$-module. Then

$$
\operatorname{ch}(V \circ W)=\operatorname{ch} V[\operatorname{ch} W]
$$

Example 2.4.9. Given $\lambda \vdash n$, let $\Pi(\lambda)$ be the set of partitions of $[n]$ whose block sizes form the partition $\lambda$. In this example, we set $\lambda=d^{b}$, where $d$ and $b$ are positive integers.

(a) The symmetric group $\mathfrak{S}_{b d}$ acts on $\Pi\left(d^{b}\right)$ as in Example 2.3.2, and this action induces a representation of $\mathfrak{S}_{b d}$ on the complex vector space $\mathbb{C} \Pi\left(d^{b}\right)$ generated by

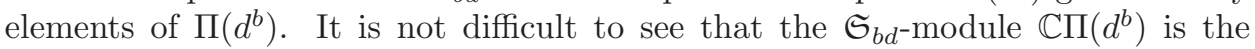
induction of the trivial representation of the stabilizer of the partition

$$
\pi\left(d^{b}\right):=1, \ldots, d / d+1, \ldots, 2 d / \ldots /(b-1) d+1, \ldots, b d
$$

to $\mathfrak{S}_{b d}$. The stabilizer of $\pi\left(d^{b}\right)$ is $\mathfrak{S}_{b}\left[\mathfrak{S}_{d}\right]$ and the trivial representation of $\mathfrak{S}_{b}\left[\mathfrak{S}_{d}\right]$ is $S^{(b)}\left[S^{(d)}\right]$. So $\mathbb{C} \Pi\left(d^{b}\right)$ is the composition product $S^{(b)} \circ S^{(d)}$. By Theorem 2.4.8

$$
\operatorname{ch} \mathbb{C} \Pi\left(d^{b}\right)=h_{b}\left[h_{d}\right] \text {. }
$$

(b) The stabilizer $\mathfrak{S}_{b}\left[\mathfrak{S}_{d}\right]$ of $\pi\left(d^{b}\right)$ acts on the interval $\left(\pi\left(d^{b}\right), \hat{1}\right)$ of $\Pi_{b d}$. This induces a representation of $\mathfrak{S}_{b}\left[\mathfrak{S}_{d}\right]$ on the top homology $\tilde{H}_{b-3}\left(\pi\left(d^{b}\right), \hat{1}\right.$ ) (recall $\tilde{H}_{i}(x, y)$ denotes complex homology of the open interval $\left.(x, y)\right)$. By observing that the interval $\left(\pi\left(d^{b}\right), \hat{1}\right)$ is isomorphic to the poset $\bar{\Pi}_{b}$, one can see that

$$
\tilde{H}_{b-3}\left(\pi\left(d^{b}\right), \hat{1}\right) \cong \mathfrak{S}_{b}\left[\mathfrak{S}_{d}\right] \tilde{H}_{b-3}\left(\bar{\Pi}_{b}\right)\left[S^{(d)}\right] .
$$

Next observe that $\bigoplus_{x \in \Pi\left(d^{b}\right)} \tilde{H}_{b-3}(x, \hat{1})$ is the induction of $\tilde{H}_{b-3}\left(\pi\left(d^{b}\right), \hat{1}\right)$ from $\mathfrak{S}_{b}\left[\mathfrak{S}_{d}\right]$ to $\mathfrak{S}_{b d}$. So $\bigoplus_{x \in \Pi\left(d^{b}\right)} \tilde{H}_{b-3}(x, \hat{1})$ is the composition product $\tilde{H}_{b-3}\left(\bar{\Pi}_{b}\right) \circ S^{(d)}$. By Theorem 2.4.8,

$$
\begin{aligned}
\operatorname{ch} \bigoplus_{x \in \Pi\left(d^{b}\right)} \tilde{H}_{b-3}(x, \hat{1}) & =\operatorname{ch} \tilde{H}_{b-3}\left(\pi\left(d^{b}\right), \hat{1}\right) \uparrow \underset{\mathfrak{S}_{b}\left[\mathfrak{S}_{d}\right]}{\mathfrak{S}_{b d}} \\
& =\left(\operatorname{ch} \tilde{H}_{b-3}\left(\bar{\Pi}_{b}\right)\right)\left[h_{d}\right] .
\end{aligned}
$$

Theorem 2.4.8 is inadequate when $\lambda$ is not of the form $d^{b}$. In $[\mathbf{1 7 5}, \mathbf{1 9 9}]$, more general results are given, which enable one to derive the following generating function,

$$
\sum_{\lambda \in \operatorname{Par}(T, b)}\left(\operatorname{ch} \bigoplus_{x \in \Pi(\lambda)} \tilde{H}_{b-3}(x, \hat{1})\right) z_{\lambda_{1}} \cdots z_{\lambda_{b}}=\left(\operatorname{ch} \tilde{H}_{b-3}\left(\bar{\Pi}_{b}\right)\right)\left[\sum_{i \in T} z_{i} h_{i}\right],
$$

where $T$ is any set of positive integers, $\operatorname{Par}(T, b)$ is the set of partitions of length $b$, all of whose parts are in $T$, the $\lambda_{i}$ are the parts of $\lambda$, and the $z_{i}$ are (commuting) indeterminates.

\subsubsection{The matching complex}

In this subsection, we further demonstrate the power of symmetric function theory and plethysm in computing homology. Our example is a well-studied simplicial complex known as the matching complex, which is defined to be the simplicial complex $M_{n}$ whose vertices are the 2-element subsets of $[n]$ and whose faces are 
collections of mutually disjoint 2-element subsets of $[n]$. Alternatively, $M_{n}$ is the simplicial complex of graphs on node set $[n]$ whose degree is at most 2 . Its face poset is the proper part of the poset of partitions of $[n]$ whose block sizes are at most 2. It is not difficult to see that

$$
\operatorname{ch} C_{k-1}\left(M_{n}\right)=e_{k}\left[h_{2}\right] h_{n-2 k} .
$$

It follows from this and the Hopf trace formula (Theorem 2.3.9) that

$$
\sum_{k \geq-1}(-1)^{k-1} \operatorname{ch} \tilde{H}_{k}\left(M_{n}\right)=\sum_{k \geq 0}(-1)^{k} e_{k}\left[h_{2}\right] h_{n-2 k},
$$

which implies by Exercise 2.4.2 that

$$
\sum_{n \geq 0} \sum_{k \geq-1}(-1)^{k-1} \operatorname{ch} \tilde{H}_{k}\left(M_{n}\right)=\prod_{i \leq j}\left(1-x_{i} x_{j}\right) \prod_{i \geq 1}\left(1-x_{i}\right)^{-1} .
$$

The right hand side can be decomposed into Schur functions by using the following symmetric function identity of Littlewood [121, p.238]:

$$
\prod_{i \leq j}\left(1-x_{i} x_{j}\right) \prod_{i \geq 1}\left(1-x_{i}\right)^{-1}=\sum_{\lambda=\lambda^{\prime}}(-1)^{\frac{|\lambda|-r(\lambda)}{2}} s_{\lambda},
$$

where $r(\lambda)$ is the rank of $\lambda$, i.e., the size of the main diagonal (or Durfee square) of the Young diagram for $\lambda$. From this we conclude that

$$
\bigoplus_{k \geq-1}(-1)^{k-1} \tilde{H}_{k}\left(M_{n}\right) \cong \mathfrak{S}_{n} \bigoplus_{\substack{\lambda: \lambda \vdash n \\ \lambda=\lambda^{\prime}}}(-1)^{\frac{|\lambda|-r(\lambda)}{2}} S^{\lambda} .
$$

With additional work involving long exact sequences of relative homology, Bouc obtains the following beautiful refinement.

Theorem 2.4.10 (Bouc $[47]$ ). For all $n \geq 1$ and $k \in \mathbb{Z}$,

$$
\tilde{H}_{k-1}\left(M_{n}\right) \cong_{\mathfrak{S}_{n}} \bigoplus_{\substack{\lambda: \lambda \vdash n \\ \lambda=\lambda^{\prime} \\ r(\lambda)}} S^{=|\lambda|-2 k}
$$

From Bouc's formula one obtains a formula for the Betti number in dimension $k-1$ as the number of standard Young tableaux of self-conjugate shape and rank $n-2 k$ (which can be computed from the hook-length formula, Theorem 2.2.5). This result provides an excellent illustration of the use of representation theory in the computation of Betti numbers.

We sketch a proof of Theorem 2.4.10 due to Dong and Wachs [62], which involves a technique called discrete Hodge theory. Let $\Delta$ be a $G$-simplicial complex. The combinatorial Laplacian $\Lambda_{k}: C_{k}(\Delta) \rightarrow C_{k}(\Delta)$ is defined by

$$
\Lambda_{k}=\delta_{k-1} \partial_{k}+\partial_{k+1} \delta_{k} .
$$

A basic result of discrete Hodge theory is that

$$
\tilde{H}_{k}(\Delta) \cong_{G} \operatorname{ker} \Lambda_{k} .
$$

The key observation of [62] is that when one applies the Laplacian $\Lambda_{k-1}$ to an oriented simplex $\gamma \in C_{k-1}\left(M_{n}\right)$, one gets

$$
\Lambda_{k-1}(\gamma)=T_{n} \cdot \gamma
$$


where $T_{n}=\sum_{1 \leq i<j \leq n}(i, j) \in \mathbb{C S}_{n}$ and $(i, j)$ denotes a transposition. It is then shown that $T_{n}$ acts on the Specht module $S^{\lambda}$ as multiplication by the scalar $c_{\lambda}$ defined by

$$
c_{\lambda}=\sum_{i=1}^{r}\left(\left(\begin{array}{c}
\alpha_{i}+1 \\
2
\end{array}\right)-\left(\begin{array}{c}
\beta_{i}+1 \\
2
\end{array}\right)\right)
$$

where $r=r(\lambda), \alpha_{i}=\lambda_{i}-i$, and $\beta_{i}=\lambda_{i}^{\prime}-i$. That is, $\alpha_{i}$ is the number of cells to the right of and in the same row as the $i$ th cell of the diagonal of $\lambda$ and $\beta_{i}$ is the number of cells below and in the same column as the $i$ th cell of the diagonal. The array $\left(\alpha_{1}, \ldots, \alpha_{r} \mid \beta_{1}, \ldots, \beta_{r}\right)$ is known as Frobenius notation for $\lambda$. Note that $\lambda$ is uniquely determined by its Frobenius notation.

Next we decompose $C_{k-1}\left(M_{n}\right)$ into Specht modules by using (2.4.4) and another symmetric function identity of Littlewood, cf., [124, I 5 Ex. 9b], namely,

$$
\prod_{i \leq j}\left(1-x_{i} x_{j}\right)=\sum_{\nu \in \mathcal{B}}(-1)^{|\nu| / 2} s_{\nu}
$$

where $\mathcal{B}$ is the set of all partitions of the form $\left(\alpha_{1}+1, \ldots, \alpha_{r}+1 \mid \alpha_{1}, \ldots, \alpha_{r}\right)$ for some $r$. This and Pieri's rule (Theorem 2.2.11) yield the decomposition into irreducibles:

$$
C_{k-1}\left(M_{n}\right) \cong \bigoplus_{\lambda \in A_{n}} a_{\lambda}^{k} S^{\lambda}
$$

where

$$
A_{n}=\left\{\left(\alpha_{1}, \ldots, \alpha_{r} \mid \beta_{1}, \ldots, \beta_{r}\right) \vdash n: r \geq 1, \alpha_{i} \geq \beta_{i} \quad \forall i \in[r]\right\}
$$

and $a_{\lambda}^{k}$ is a nonnegative integer. If $\lambda$ is self-conjugate then

$$
a_{\lambda}^{k}=\left\{\begin{array}{ll}
1 & \text { if } r(\lambda)=n-2 k \\
0 & \text { otherwise }
\end{array} .\right.
$$

Clearly for $\lambda \in A_{n}$, we have $c_{\lambda}=0$ if and only if $\lambda$ is self-conjugate. It follows that

$$
\operatorname{ker} \Lambda_{p-1} \cong \bigoplus_{\substack{\lambda: \lambda \vdash n \\
\lambda=\lambda^{\prime}}} a_{\lambda}^{k} S^{\lambda} \cong \bigoplus_{\begin{array}{c}
\lambda: \lambda \vdash n \\
\lambda=\lambda^{\prime} \\
r(\lambda)=n-2 k
\end{array}} S^{\lambda} .
$$

Theorem 2.4.10 now follows by discrete Hodge theory (2.4.6).

There is a bipartite analog of the matching complex called the $m \times n$ chessboard complex. This is the simplicial complex of subgraphs of the complete bipartite graph on node sets of size $m$ and $n$, whose nodes have degree at most 1 . Alternatively, it is the simplicial complex of nonattacking rook placements on an $m \times n$ chessboard. Friedman and Hanlon [75] use discrete Hodge theory to obtain a chessboard complex analog of Bouc's formula (2.4.5). (In fact the proof of (2.4.5) described above was patterned on the Friedman-Hanlon proof.) Not only can the Betti numbers be computed from this formula, but Shareshian and Wachs [155] use the formula to compute torsion in the integral homology of the chessboard complex; see Theorem 5.5.5. So representation theory has proved to be a useful tool in computing torsion in integral homology as well as Betti numbers. Karaguezian, Reiner and Wachs [105] use formula (2.4.5) and its chessboard complex analog to derive a more general result of Reiner and Roberts [138], which computes the homology of general bounded degree graph and bipartite graph complexes. 
The matching complex and chessboard complex have arisen in various areas of mathematics, such as group theory (see Section 5.2), commutative algebra [138] and discrete geometry [221]. These complexes are discussed further in Lecture 5. See [200] for a survey article on the topology of matching complexes, chessboard complexes and general bounded degree graph complexes. 



\section{LECTURE 3 Shellability and edge labelings}

\subsection{Shellable simplicial complexes}

Shellability is a combinatorial property of simplicial and more general cell complexes, with strong topological and algebraic consequences. Shellability first appeared in the middle of the nineteenth century in Schläfli's computation of the Euler characteristic of a convex polytope [150]. Schläfli made the assumption, without proof, that the boundary complex of a convex polytope is shellable. This assumption was eventually proved in 1970 by Brugesser and Mani [52] and was used in McMullen's proof [126] of the famous upper bound conjecture for convex polytopes (the upper bound conjecture is stated in Section 4.1).

The original theory of shellability applied only to pure complexes. In the early 1990's, a nonpure simplicial complex arose in the complexity theory work of Björner, Lovász and Yao [34] with topological properties somewhat similar to those of pure shellable complexes. This led Björner and Wachs $[\mathbf{4 0 , 4 1 ]}$ to extend the theory of shellability to nonpure complexes. The Goresky-MacPherson formula created a need for such an extension, since, unlike for hyperplane arrangements, the intersection semilattice of a subspace arrangement is not necessarily pure. As it turned out, there were many other uses for nonpure shellability.

For each face $F$ of a simplicial complex $\Delta$, let $\langle F\rangle$ denote the subcomplex generated by $F$, i.e., $\langle F\rangle=\{G: G \subseteq F\}$. A simplicial complex $\Delta$ is said to be shellable if its facets can be arranged in linear order $F_{1}, F_{2}, \ldots, F_{t}$ in such a way that the subcomplex $\left(\bigcup_{i=1}^{k-1}\left\langle F_{i}\right\rangle\right) \cap\left\langle F_{k}\right\rangle$ is pure and ( $\left.\operatorname{dim} F_{k}-1\right)$-dimensional for all $k=2, \ldots, t$. Such an ordering of facets is called a shelling. We emphasize that we are not assuming purity; here shellability refers to what is commonly called nonpure shellability.

A shelling of the boundary complex of the 3-simplex is given in Figure 3.1.1. A pure nonshellable complex and a nonpure shellable complex are given in Figure 3.1.2, where the shading indicates that the triangles are filled in. 

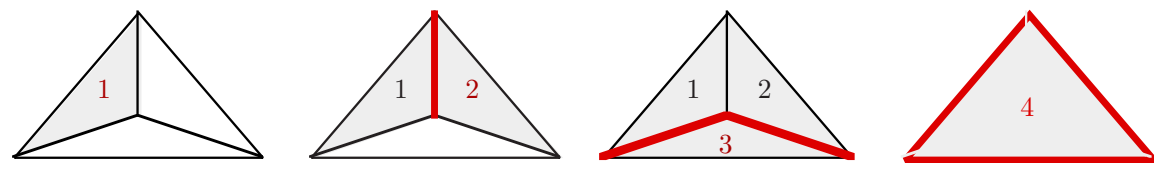

Figure 3.1.1. Shelling of the boundary of 3-simplex
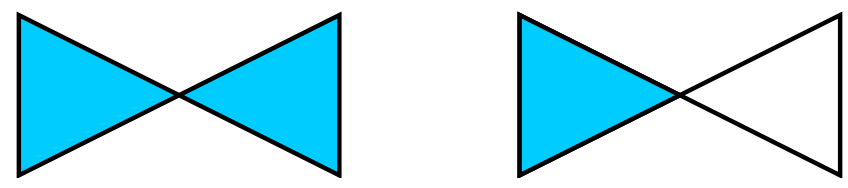

Figure 3.1.2

Exercise 3.1.1. Verify that the third and fifth simplicial complexes below are not shellable, while the others are.
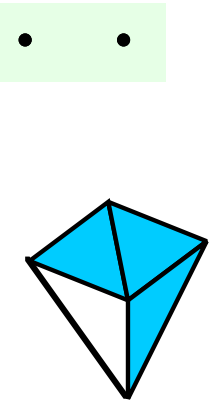
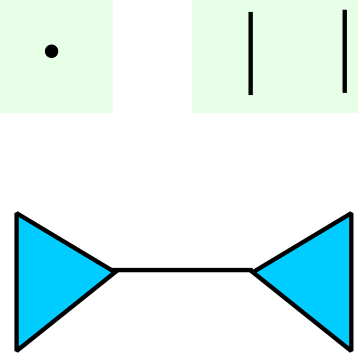

Theorem 3.1.2 (Brugesser and Mani [52]). The boundary complex of a convex polytope is shellable.

A geometric construction called a line shelling is used to prove this result. See Ziegler's book [218] on polytopes for a description of this basic construction.

Shellability is not a topological property. By this we mean that shellability of a complex is not determined by the topology of its geometric realization; a shellable simplicial complex can be homeomorphic to a nonshellable simplicial complex. In fact, there exist nonshellable triangulations of the 3-ball and the 3 -sphere; see [118],[218, Chapter 8], [219], [123]. 
Shellability does have strong topological consequences, however, as is shown by the following result. By a wedge $\vee_{i=1}^{n} X_{i}$ of $n$ mutually disjoint connected topological spaces $X_{i}$, we mean the space obtained by selecting a base point for each $X_{i}$ and then identifying all the base points with each other.

Theorem 3.1.3 (Björner and Wachs [40]). A shellable simplicial complex has the homotopy type of a wedge of spheres (in varying dimensions), where for each $i$, the number of $i$-spheres is the number of $i$-facets whose entire boundary is contained in the union of the earlier facets. Such facets are usually called homology facets.

Proof idea. Let $\Delta$ be a shellable simplicial complex. We first observe that any shelling of $\Delta$ can be rearranged to produce a shelling in which the homology facets come last. So $\Delta$ has a shelling $F_{1}, F_{2}, \ldots F_{k}$ in which $F_{1}, \ldots, F_{j}$ are not homology facets and $F_{j+1}, \ldots, F_{k}$ are, where $1 \leq j \leq k$. The basic idea of the proof is that as we attach the first $j$ facets, we construct a contractible simplicial complex at each step. The remaining homology facets attach as spheres since the entire boundary of the facet is identified with a point.

Corollary 3.1.4. If $\Delta$ is shellable then for all $i$,

$$
\tilde{H}_{i}(\Delta ; \mathbb{Z}) \cong \tilde{H}^{i}(\Delta ; \mathbb{Z}) \cong \mathbb{Z}^{r_{i}},
$$

where $r_{i}$ is the number of homology i-facets of $\Delta$.

The homology facets yield more than just the Betti numbers; they form a basis for cohomology. We discuss the connection with cohomology further in the context of lexicographic shellability in the next section.

There are certain operations on complexes and posets that preserve shellability. For a simplicial complex $\Delta$ and $F \in \Delta$, define the link to be the subcomplex given by

$$
\mathrm{lk}_{\Delta} F:=\{G \in \Delta: G \cup F \in \Delta, G \cap F=\emptyset\} .
$$

Theorem 3.1.5 ([40]). The link of every face of a shellable complex is shellable.

Define the suspension of a simplicial complex $\Delta$ to be

$$
\operatorname{susp}(\Delta):=\Delta *\{\{a\},\{b\}\},
$$

where $*$ denotes the join, and $a \neq b$ are not vertices of $\Delta$.

Theorem 3.1.6 ([40]). The join of two simplicial complexes is shellable if and only if each complex is shellable. In particular, $\operatorname{susp}(\Delta)$ is shellable if and only if $\Delta$ is shellable.

Define the $k$-skeleton of a simplicial complex $\Delta$ to be the subcomplex consisting of all faces of dimension $k$ or less.

Theorem 3.1.7 ([41]). The $k$-skeleton of a shellable simplicial complex is shellable for all $k \geq 0$.

A simplicial complex $\Delta$ is said to be $r$-connected (for $r \geq 0$ ) if it is nonempty and connected and its $j$ th homotopy group $\pi_{j}(\Delta)$ is trivial for all $j=1, \ldots, r$. So 0 -connected is the same as connected and 1-connected is the same as simply connected. A nonempty simplicial complex $\Delta$ is said to be $r$-acyclic if its $j$ th reduced integral homology group $\tilde{H}_{j}(\Delta)$ is trivial for all $j=0,1, \ldots, r$. We say that $X$ is $(-1)$-connected and $(-1)$-acyclic when $\Delta$ is nonempty. It is also convenient to say that every simplicial complex is $r$-connected and $r$-acyclic for all $r \leq-2$. 
It is a basic fact of homotopy theory that $r$-connected implies $r$-acyclic and that the converse holds only for simply connected complexes. Another basic fact is that a a simplicial complex is $r$-connected ( $r$-acyclic) if and only if its $(r+1)$ skeleton has the homotopy type (homology) of a wedge of $(r+1)$-spheres. This makes shellability a useful tool in establishing $r$-connectivity and $r$-acyclicity.

Theorem 3.1.8. A simplicial complex is $r$-connected (and therefore $r$-acyclic) if its $(r+1)$-skeleton is pure shellable.

A poset $P$ is said to be shellable if its order complex $\Delta(P)$ is shellable. The following is an immediate consequence of Theorems 3.1.5 and 3.1.6.

\section{Corollary 3.1.9.}

(a) A bounded poset $P$ is shellable if and only if its proper part $\bar{P}$ is shellable.

(b) Every (open or closed) interval of a shellable poset is shellable.

(c) The join of two posets is shellable if and only if each of the posets is shellable.

Theorem 3.1.10 (Björner and Wachs [41]). The product of bounded posets is shellable if and only if each of the posets is shellable.

Sometimes shellability has stronger topological consequences than homotopy type.

Theorem 3.1.11 (Danaraj and Klee [56]). Let $\Delta$ be a pure shellable d-dimensional simplicial complex in which every codimension 1 face is contained in at most 2 facets. Then $\Delta$ is homeomorphic to a d-sphere or a d-ball. Moreover, $\Delta$ is homeomorphic to a d-sphere if and only if every codimension 1 face is contained in exactly 2 facets.

Note that the condition on codimension 1 faces in the Danaraj and Klee theorem can be expressed as follows: Closed length 2 intervals of $L(\Delta)$ have at most 4 elements. Pure posets in which every length 2 interval has exactly 4 elements are said to be thin. We have the following poset version of the Danaraj and Klee result.

Theorem 3.1.12 (Björner [23]). If $\hat{P}$ is pure, thin and shellable then $P$ is isomorphic to the face poset of a regular cell decomposition (a generalization of simplicial decomposition; see $[\mathbf{9 2}])$ of an $l(P)$-sphere.

Many natural classes of simplicial complexes and posets, which have arisen in various fields of mathematics, have turned out to be shellable (pure or nonpure). A striking illustration of the ubiquity of shellability is given by the following result.

Theorem 3.1.13 (Shareshian [151]). The lattice of subgroups of a finite group $G$ is shellable if and only if $G$ is solvable.

The pure version of this result, which states that the lattice of subgroups of a finite group $G$ is pure shellable if and only if $G$ is supersolvable, was proved by Björner [21] in the late 70's by introducing a technique called lexicographic shellability. Shareshian uses a general (nonpure) version of lexicographic shellability more recently introduced by Björner and Wachs [40]. We discuss lexicographic shellability in the remaining sections of this lecture. Further discussion of connections between group theory and poset topology can be found in Section 5.2.

We end this section with two interesting conjectures. A pure simplicial complex $\Delta$ is said to be extendably shellable if every partial shelling of $\Delta$ extends to a shelling 
of $\Delta$. Tverberg (see [56]) conjectured that the boundary complex of every polytope is extendably shellable. The conjecture was settled in the negative by Ziegler [219] who showed that there are simple and simplicial polytopes whose boundary complex is not extendably shellable. The conjecture is, however, true for the simplex since every ordering of the facets of the boundary complex is a shelling. Hence the $k=d-1$ case of the following conjecture holds.

Conjecture 3.1.14 (Simon $[\mathbf{1 6 0}]$ ). For all $k \leq d$, the $k$-skeleton of the $d$-simplex is extendably shellable.

Simon's conjecture was shown to be true for $k \leq 2$ by Björner and Eriksson [31], who extended the conjecture to all matroid complexes. Recently Hall [84] showed that the boundary complex of the 12-dimensional cross-polytope is a counterexample to the extended conjecture of Björner and Eriksson.

A simplicial complex $\Delta$ is said to be minimally nonshellable (called an obstruction to shellability in [198]) if $\Delta$ is not shellable, but every proper induced subcomplex of $\Delta$ is shellable. For example, the complex consisting of two disjoint 1-simplexes is a minimally nonshellable complex (see Exercise 3.1.1). Billera and Myers [20] showed that this is the only minimally nonshellable order complex. It is also the only 1-dimensional minimally nonshellable simplicial complex. It was shown by Wachs [198] that the number of vertices in any 2-dimensional minimally nonshellable simplicial complex is either 5,6 or 7 ; hence there are only finitely many such complexes.

Conjecture 3.1.15 (Wachs). There are only finitely many d-dimensional minimally nonshellable simplicial complexes (obstructions to shellability) for each $d$.

\subsection{Lexicographic shellability}

In the early 1970's, Stanley $[\mathbf{1 6 3}, \mathbf{1 6 4}]$ introduced a technique for showing that the Möbius function of rank-selected subposets of certain posets alternates in sign. This technique involved labeling the edges of the Hasse diagram of the poset in a certain way. Stanley conjectured that the posets that he was considering were Cohen-Macaulay, a topological (and algebraic) property of simplicial complexes implied by shellability (cf., Section 4.1). Björner [21] proved this conjecture by finding a condition on edge labelings which implies shellability of the poset. From this emerged the theory of lexicographic shellability, which was further developed in a series of papers by Björner and Wachs, first in the pure case $[\mathbf{3 7}, \mathbf{3 8}]$ and later in the general (nonpure) case $[\mathbf{4 0}, \mathbf{4 1}]$.

There are two basic versions of lexicographic shellability, EL-shellability and CL-shellability. In this section we begin with the simpler but less powerful version, EL-shellability, and discuss some of its consequences.

An edge labeling of a bounded poset $P$ is a map $\lambda: \mathcal{E}(P) \rightarrow \Lambda$, where $\mathcal{E}(P)$ is the set of edges of the Hasse diagram of $P$, i.e., the covering relations $x \lessdot y$ of $P$, and $\Lambda$ is some poset (usually the integers $\mathbb{Z}$ with its natural total order relation). Given an edge labeling $\lambda: \mathcal{E}(P) \rightarrow \Lambda$, one can associate a word

$$
\lambda(c)=\lambda\left(\hat{0}, x_{1}\right) \lambda\left(x_{1}, x_{2}\right) \cdots \lambda\left(x_{t}, \hat{1}\right)
$$

with each maximal chain $c=\left(\hat{0} \lessdot x_{1} \lessdot \cdots \lessdot x_{t} \lessdot \hat{1}\right)$. We say that $c$ is increasing if the associated word $\lambda(c)$ is strictly increasing. That is, $c$ is increasing if

$$
\lambda\left(\hat{0}, x_{1}\right)<\lambda\left(x_{1}, x_{2}\right)<\cdots<\lambda\left(x_{t}, \hat{1}\right) .
$$




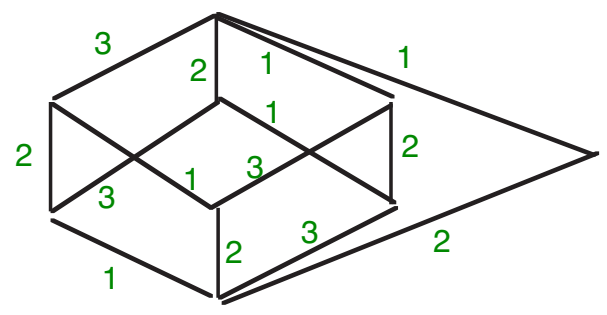

Figure 3.2.1. EL-labeling

We say that $c$ is decreasing if the associated word $\lambda(c)$ is weakly increasing. We can order the maximal chains lexicographically by using the lexicographic order on the corresponding words. Any edge labeling $\lambda$ of $P$ restricts to an edge labeling of any closed interval $[x, y]$ of $P$. So we may refer to increasing and decreasing maximal chains of $[x, y]$, and lexicographic order of maximal chains of $[x, y]$.

Definition 3.2.1. Let $P$ be a bounded poset. An edge-lexicographical labeling (ELlabeling, for short) of $P$ is an edge labeling such that in each closed interval $[x, y]$ of $P$, there is a unique increasing maximal chain, which lexicographically precedes all other maximal chains of $[x, y]$.

An example of an EL-labeling of a poset is given in Figure 3.2.1. The leftmost chain, which has associated word 123, is the only increasing maximal chain of the interval $[\hat{0}, \hat{1}]$. It is also lexicographically less than all other maximal chains. One needs to check each interval to verify that the labeling is indeed an EL-labeling.

A bounded poset that admits an EL-labeling is said to be edge-lexicographic shellable (EL-shellable, for short). The following theorem justifies the name.

Theorem 3.2.2 (Björner [21], Björner and Wachs [40]). Suppose $P$ is a bounded poset with an EL-labeling. Then the lexicographic order of the maximal chains of $P$ is a shelling of $\Delta(P)$. Moreover, the corresponding order of the maximal chains of $\bar{P}$ is a shelling of $\Delta(\bar{P})$.

Exercise 3.2.3. Prove Theorem 3.2.2.

It is for nonbounded posets that shellability has interesting topological consequences since the order complex of a bounded poset is a just a cone.

Theorem 3.2.4 (Björner and Wachs [40]). Suppose $P$ is a poset for which $\hat{P}$ admits an EL-labeling. Then $P$ has the homotopy type of a wedge of spheres, where the number of $i$-spheres is the number of decreasing maximal $(i+2)$-chains of $\hat{P}$. The decreasing maximal $(i+2)$-chains, with $\hat{0}$ and $\hat{1}$ removed, form a basis for cohomology $\tilde{H}^{i}(P ; \mathbb{Z})$.

The first part of Theorem 3.2.4 is a consequence of Theorems 3.1.3 and 3.2.2. Indeed, the proper parts of the decreasing chains are the homology facets of the shelling of $\Delta(P)$ induced by the lexicographic order of maximal chains of $\hat{P}$. To establish the second part, one needs only show that the proper parts of the decreasing chains span cohomology. This is proved by showing that the cohomology relations enable one to express a maximal chain with an ascent as the negative of a sum 


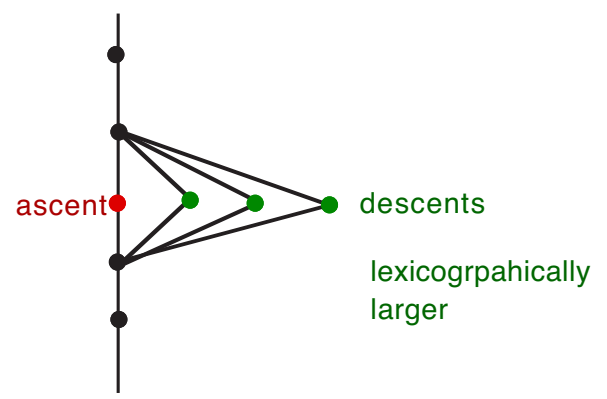

Figure 3.2.2. Straightening step

of lexicographically larger maximal chains. This provides a step in a "straightening" algorithm for expressing maximum chains as linear combinations of decreasing maximum chains. See Figure 3.2.2.

Remark 3.2.5. In Björner's original version of EL-shellability, the unique increasing maximal chain was required to be weakly increasing and the decreasing chains were required to be strictly decreasing. The two versions have the same topological and algebraic consequences, but it is unknown whether they are equivalent.

Example 3.2.6. In the EL-labeling given in Figure 3.2.1, the two rightmost maximal chains are the only decreasing maximal chains. One has length 3 and the other has length 2. It follows form Theorem 3.2.4 that the order complex of the proper part of the poset has the homotopy type of a wedge of a 1-sphere and a 0 -sphere. This is consistent with the fact that the order complex of the proper part of the poset consists of the barycentric subdivision of the boundary of a 2-simplex and an isolated point.

\subsubsection{The Boolean algebra $B_{n}$.}

There is a very natural EL-labeling of the Boolean algebra $B_{n}$; simply label the covering relation $A_{1} \subset A_{2}$ with the unique element in the singleton set $A_{2}-A_{1}$. The maximal chains correspond to the permutations in $\mathfrak{S}_{n}$. It is easy to see that each interval has a unique increasing chain that is lexicographically first. There is only one decreasing chain which is consistent with the fact that $\Delta\left(\bar{B}_{n}\right)$ is a sphere. See Figure 3.2.3.

For each $k \leq n$, define the truncated Boolean algebra $B_{n}^{k}$ to be the subposet of $B_{n}$ given by

$$
B_{n}^{k}=\{A \subseteq[n]:|A| \geq k\} .
$$

Define an edge labeling $\lambda$ of $B_{n}^{k} \cup\{\hat{0}\}$ as follows:

$$
\lambda\left(A_{1}, A_{2}\right)= \begin{cases}\max A_{2} & \text { if } A_{1}=\hat{0} \text { and }\left|A_{2}\right|=k \\ a & \text { if } A_{2}-A_{1}=\{a\} .\end{cases}
$$

It is easy to check that this is an EL-labeling. The decreasing chains correspond to permutations with descent set $\{k, k+1, \ldots, n-1\}$. Hence $\operatorname{dim} \tilde{H}^{n-k-1}\left(\bar{B}_{n}^{k}\right)$ equals the number of permutations in $\mathfrak{S}_{n}$ with descent set $\{k, \ldots, n-1\}$, which equals the number of standard Young tableaux of hook shape $k 1^{n-k}$. An equivariant version of 


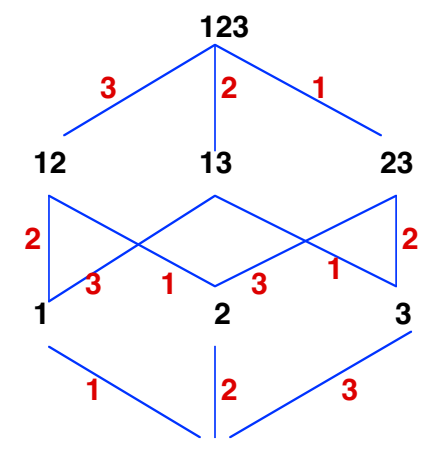

Figure 3.2.3. EL-labeling of $B_{3}$

this result is given by the following special case of a result of Solomon (the general result is given in Section 3.4).

Theorem 3.2.7 (Solomon [161]). For all $k \leq n$,

$$
\tilde{H}_{n-k-1}\left(\bar{B}_{n}^{k}\right) \cong_{\mathfrak{S}_{n}} S^{\left(k 1^{n-k}\right)} .
$$

Combinatorial proof idea. We use a surjection from the set of tableaux of shape $k 1^{n-k}$ to the set of maximal chains of $\bar{B}_{n}^{k}$, illustrated by the following example in which $n=5$ and $k=2$.

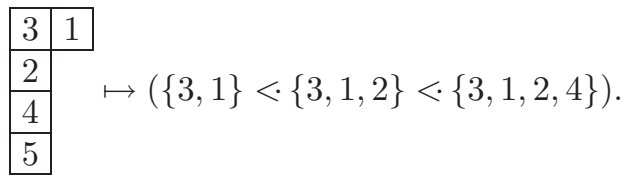

The surjection determines a surjective $\mathfrak{S}_{n}$-homomorphism from the tableaux module $M^{\lambda}$ to the chain space $C_{n-k-2}\left(\bar{B}_{n}^{k} ; \mathbb{C}\right)$. To show that this homomorphism induces a surjective homomorphism from the quotient space $S^{\lambda}$ to the quotient space $\tilde{H}^{n-k-2}\left(\bar{B}_{n}^{k}\right)=C_{n-k-2}\left(\bar{B}_{n}^{k} ; \mathbb{C}\right) / B^{n-k-2}\left(\bar{B}_{n}^{k} ; \mathbb{C}\right)$, we observe that the row relations map to 0 in $C_{n-k-2}\left(\bar{B}_{n}^{k} ; \mathbb{C}\right)$ and show that the Garnir relations map to coboundary relations. Since the dimensions of the two vector spaces are equal, the homomorphism is an isomorphism. We demonstrate the fact that Garnir relations map to cohomology relations on two examples and leave the general proof as an exercise. The Garnir relation

$$
\begin{array}{|l|l|}
\hline 3 & 1 \\
\cline { 1 - 1 } 2 &
\end{array}+\begin{array}{|l|l|}
\hline 3 & 1 \\
\hline 4 & \\
\hline 4 & \\
\hline & \\
\hline
\end{array}
$$

maps to the sum of maximal chains of the proper part of the poset

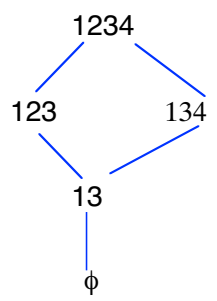


The Garnir relation

$$
\begin{array}{|l|l|}
\hline 3 & 1 \\
\hline 2 & \\
\hline 4 & +
\end{array}+\begin{array}{|l|l|}
\hline 2 & 1 \\
\hline 3 & \\
\hline 4 & \\
\hline
\end{array} \quad+\begin{array}{|l|l|}
\hline 2 & 3 \\
\hline 1 & \\
\hline 4 & \\
\hline
\end{array}
$$

maps to the sum of maximal chains of the proper part of the poset

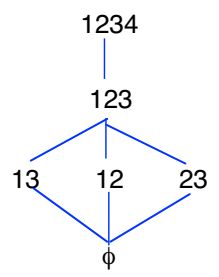

\subsubsection{The partition lattice $\Pi_{n}$.}

We give two different EL-labelings of the partition lattice. The first one, due to Gessel, appears in [21], and the second one is due to Wachs [196]. If $x<y$ in $\Pi_{n}$ then $y$ is obtained from $x$ by merging two blocks, say $B_{1}$ and $B_{2}$. For the first edge labeling $\lambda_{1}$, let

$$
\lambda_{1}(x, y)=\max \left\{\min B_{1}, \min B_{2}\right\}
$$

and for the second edge labeling $\lambda_{2}$, let

$$
\lambda_{2}(x, y)=\max B_{1} \cup B_{2} .
$$

The increasing chain from $\hat{0}$ to $\hat{1}$ is the same for both labelings; it consists of partitions with only one nonsingleton block. More precisely, the chain is given by

$$
\hat{0}<\cdot\{1,2\}<\{1,2,3\}<\cdots<\hat{1},
$$

where we have written only the nonsingleton block of each partition in the chain. We leave it as an exercise to show that these labelings are EL-labelings of the partition lattice.

The decreasing maximal chains for $\lambda_{1}$ and $\lambda_{2}$ are not the same. We describe those for $\lambda_{2}$ first. They also consist of partitions with only one nonsingleton block and are of the form

$$
\begin{aligned}
c_{\sigma}:=(\hat{0}< & <\sigma(n), \sigma(n-1)\}<\{\sigma(n), \sigma(n-1), \sigma(n-2)\}<\cdots \\
& <\{\sigma(n), \sigma(n-1), \sigma(n-2), \ldots, \sigma(1)\}=\hat{1}),
\end{aligned}
$$

where $\sigma \in \mathfrak{S}_{n}$ and $\sigma(n)=n$. We conclude that the homotopy type of $\bar{\Pi}_{n}$ is given by

$$
\bar{\Pi}_{n} \simeq \bigvee_{(n-1) !} \mathbb{S}^{n-3}
$$

and that the chains $\bar{c}_{\sigma}$, where $\sigma \in \mathfrak{S}_{n}$ and $\sigma(n)=n$, form a basis for $\tilde{H}^{n-3}\left(\bar{\Pi}_{n}, \mathbb{Z}\right)$. From this basis one can immediately see that

$$
\tilde{H}_{n-3}\left(\Pi_{n}\right) \downarrow_{\mathfrak{S}_{n-1}}^{\mathfrak{S}_{n}} \cong \mathfrak{S}_{n-1} \quad \tilde{H}^{n-3}\left(\Pi_{n}\right) \downarrow_{\mathfrak{S}_{n-1}}^{\mathfrak{S}_{n}} \cong \mathfrak{S}_{n-1} \quad \mathbb{C S}_{n-1},
$$

a result obtained by Stanley [167] as a consequence of his formula for the full representation of $\mathfrak{S}_{n}$ on $\tilde{H}_{n-3}\left(\bar{\Pi}_{n}\right)$ (Theorem 4.4.7). 


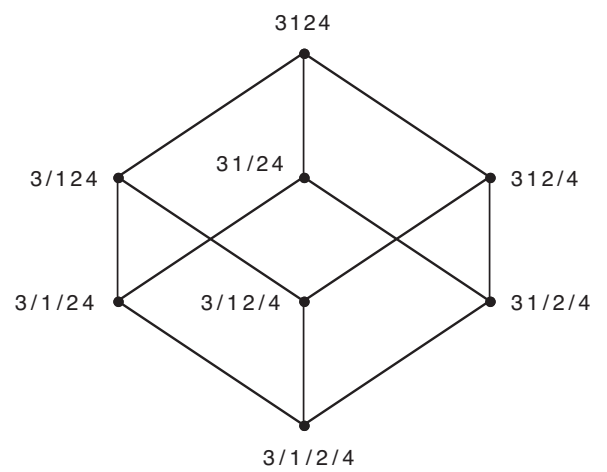

Figure 3.2.4. $\Pi_{3124}$

Next we describe a nice basis for homology of $\bar{\Pi}_{n}$ that is dual to the decreasing chain basis for $\lambda_{2}$. To split a permutation $\sigma \in \mathfrak{S}_{n}$ at positions $j_{1}<j_{2}<\cdots<j_{k}$ in $[n-1]$ is to form the partition

$\sigma(1), \sigma(2), \ldots, \sigma\left(j_{1}\right) / \sigma\left(j_{1}+1\right), \sigma\left(j_{1}+2\right), \ldots, \sigma\left(j_{2}\right) / \ldots / \sigma\left(j_{k}+1\right), \sigma\left(j_{k}+2\right), \ldots, \sigma(n)$

of $[n]$. For each $\sigma \in \mathfrak{S}_{n}$, let $\Pi_{\sigma}$ be the induced subposet of the partition lattice $\Pi_{n}$ consisting of partitions obtained by splitting the permutation $\sigma$ at any set of positions in $[n-1]$. The subposet $\Pi_{3124}$ of $\Pi_{4}$ is shown in Figure 3.2.4. Each poset $\Pi_{\sigma}$ is isomorphic to the subset lattice $B_{n-1}$. Therefore $\Delta\left(\bar{\Pi}_{\sigma}\right)$ is an $(n-3)$ sphere embedded in $\Delta\left(\bar{\Pi}_{n}\right)$, and hence it determines a fundamental cycle $\rho_{\sigma} \in$ $\tilde{H}_{n-3}\left(\bar{\Pi}_{n} ; \mathbb{Z}\right)$.

Theorem 3.2.8 (Wachs [196]). The set $\left\{\rho_{\sigma}: \sigma \in \mathfrak{S}_{n}, \sigma(n)=n\right\}$ forms a basis for $\tilde{H}_{n-3}\left(\bar{\Pi}_{n} ; \mathbb{Z}\right)$ dual to the decreasing chain basis $\left\{\bar{c}_{\sigma}: \sigma \in \mathfrak{S}_{n}, \sigma(n)=n\right\}$ for cohomology. Call the homology basis, the splitting basis.

Now we describe the decreasing chain basis for cohomology for the EL-labeling $\lambda_{1}$ and its dual basis for homology. Given any rooted nonplanar (i.e. children of a node are unordered) tree $T$ on node set $[n]$, by removing any set of edges of $T$, one forms a partition of $[n]$ whose blocks are the node sets of the connected components of the resulting graph. Let $\Pi_{T}$ be the induced subposet of the partition lattice $\Pi_{n}$ consisting of partitions obtained by removing edges of $T$; see Figure 3.2.5. (If $T$ is a linear tree then $\Pi_{T}$ is the same as $\Pi_{\sigma}$, where $\sigma$ is the permutation obtained by reading the nodes of the tree from the root down.) Each poset $\Pi_{T}$ is isomorphic to the subset lattice $B_{n-1}$. We let $\rho_{T}$ be the fundamental cycle of the spherical complex $\Delta\left(\bar{\Pi}_{T}\right)$. Let $T$ be an increasing tree on node set $[n]$, i.e., a rooted nonplanar tree on node set $[n]$ in which each node $i$ is greater than its parent $p(i)$. We form the chain $c_{T}$ in $\Pi_{T}$, from top down, by removing the edges $\{i, p(i)\}$, one at a time, in increasing order of $i$. For the increasing tree $T$ in Figure 3.2.5,

$$
c_{T}=(1 / 2 / 3 / 4<3 / 24 / 1<324 / 1<1234) .
$$

We claim that the $c_{T}$, where $T$ is an increasing tree on node set $[n]$, are the decreasing chains of $\lambda_{1}$. 

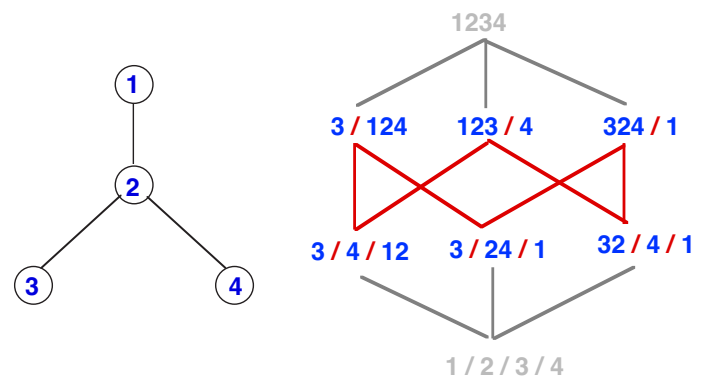

Figure 3.2.5. $T$ and $\Pi_{T}$

Theorem 3.2.9. Let $\mathcal{T}_{n}$ be the set of increasing trees on node set $[n]$. The set $\left\{\rho_{T}: T \in \mathcal{T}_{n}\right\}$ forms a basis for $\tilde{H}_{n-3}\left(\bar{\Pi}_{n} ; \mathbb{Z}\right)$ dual to the decreasing chain basis $\left\{\bar{c}_{T}: T \in \mathcal{T}_{n}\right\}$ for cohomology. Call the homology basis, the tree splitting basis.

\section{Exercise 3.2.10.}

(a) Show $\lambda_{1}$ is an EL-labeling whose decreasing maximal chains are of the form $c_{T}$, where $T$ is an increasing tree.

(b) Prove Theorem 3.2.9

(c) Show $\lambda_{2}$ is an EL-labeling whose decreasing maximal chains are of the form $c_{\sigma}$, where $\sigma \in \mathfrak{S}_{n}$ is such that $\sigma(n)=n$.

(d) Prove Theorem 3.2.8.

Both of the EL-labelings and their corresponding bases are special cases of more general constructions. The first EL-labeling $\lambda_{1}$ and its bases are specializations of constructions for geometric lattices due to Björner $[\mathbf{2 1}, \mathbf{2 2}]$; this is discussed in the next section. The second EL-labeling $\lambda_{2}$ generalizes to all Dowling lattices; see the next exercise. A geometric interpretation of the splitting basis, in which the fundamental cycles correspond to bounded regions in an affine slice of the real braid arrangement, is given by Björner and Wachs [42]. This leads to analogs of the splitting basis for intersection lattices of other Coxeter arrangements, in particular the type B partition lattice $\Pi_{n}^{B}$. An analog of the splitting basis for all Dowling lattices is given by Gottlieb and Wachs [83].

Exercise 3.2.11 (Gottlieb [81, Section 7.3]).

(a) Find an EL-labeling of the type $B$ partition lattice $\Pi_{n}^{B}$ analogous to the EL-labeling $\lambda_{2}$ of $\Pi_{n}$.

(b) Describe the decreasing chains in terms of signed permutations in $\mathfrak{S}_{n}\left[\mathbb{Z}_{2}\right]$ whose right-to-left maxima are positive, where for $i=1,2, \ldots n$, we say that $\sigma(i)$ is a right-to-left maxima of $\sigma$ if $|\sigma(i)|>|\sigma(j)|$ for all $j=i \ldots n$.

(c) Show that the number of decreasing chains is $(2 n-1) ! !:=1 \cdot 3 \cdots(2 n-1)$, thereby recovering the well-known fact that $\bar{\Pi}_{n}^{B}$ has the homotopy type of a wedge of $(2 n-1) ! !-$ spheres of dimension $n-2$.

A partition $\pi \in \Pi_{n}$ is said to be noncrossing if for all $a<b<c<d$, whenever $a, c$ are in a block $B$ of $\pi$ and $b, d$ are in a block $B^{\prime}$ of $\pi$ then $B=B^{\prime}$. Let $\mathrm{NC}_{n}$ be the induced subposet of $\Pi_{n}$ consisting of noncrossing partitions. This poset, known as the noncrossing partition lattice, was first introduced by Kreweras [113] who 
showed that it is a graded lattice with Möbius invariant equal to the signed Catalan number $(-1)^{n-1} \frac{1}{n}\left(\begin{array}{c}2 n-2 \\ n-1\end{array}\right)$. It has since undergone extensive study due to its many fascinating properties and its connections to various diverse fields of mathematics such as combinatorics, discrete geometry, mathematical biology, geometric group theory, low dimensional topology, and free probability; see the survey papers of Simion [159] and McCammond [125].

Björner and Edelman showed that $\mathrm{NC}_{n}$ is EL-shellable (see Exercise 3.2.12). Reiner [137] introduced an analog of the noncrossing partition lattice for the type $\mathrm{B}$ Coxeter group, and established many of the same properties held by $\mathrm{NC}_{n}$, including EL-shellability. A generalization of the types A and B noncrossing partition lattices to all finite Coxeter groups was recently introduced in the geometric group theory /low dimensional topology work of Bessis [17] and Brady and Watt [49]. This generalization is discussed in Section 3.3. The Möbius function for the type D noncrossing partition lattice was computed by Athanasiadis and Reiner and the question of EL-shellability was raised [7]. The general Coxeter group noncrossing partition lattices were subsequently shown to be EL-shellable by Athanasiadis, Brady and Watt [6].

Exercise 3.2.12 (Björner and Edelman, cf. [21]).

(a) Show that the restriction to $\mathrm{NC}_{n}$ of one of the EL-labelings of $\Pi_{n}$ described above is an EL-labeling.

(b) Describe the decreasing chains for the EL-labeling and show that their number is the Catalan number $\frac{1}{n}\left(\begin{array}{c}2 n-2 \\ n-1\end{array}\right)$.

Exercise 3.2.13 (Stanley [171]). Define an edge labeling for $\mathrm{NC}_{n}$ by

$$
\lambda(x, y)=\max \left\{i \in B_{1}: i<\min B_{2}\right\},
$$

where $B_{1}$ and $B_{2}$ are the blocks of $x$ that are merged to obtain $y$ and $\min B_{1}<$ $\min B_{2}$.

(a) Show that $\lambda$ is an EL-labeling of $\mathrm{NC}_{n}$.

(b) Show that the decreasing chains correspond bijectively, via the labeling, to the sequences of the form $\left(a_{1} \geq a_{2} \geq \cdots \geq a_{n-1}\right)$ where $a_{n-i} \in[i]$ for all $i \in[n-1]$. Show that the number of such sequences is a Catalan number.

This labeling is particularly interesting because the maximal chains correspond bijectively, via this labeling, to a well-studied class of sequences called parking functions; see [171].

\subsubsection{Geometric lattices}

A geometric lattice is a lattice $L$ that is semimodular (which means that for all $x, y \in L$, the join $x \vee y$ covers $y$ whenever $x$ covers the meet $x \wedge y$ ) and atomic (which means every element of $L$ is the join of atoms). Some fundamental examples are the subset lattice $B_{n}$, the subspace lattice $B_{n}(q)$, the partition lattice $\Pi_{n}$, the type $B$ partition lattice $\Pi_{n}^{B}$, and more generally, the intersection lattice $L(\mathcal{A})$ of any central hyperplane arrangement. Geometric lattices are fundamental structures of matroid theory. It is easy to show that geometric lattices are pure.

We describe an edge labeling for geometric lattices, which comes from Stanley's early work [164] on rank-selected Möbius invariants and was one of the main motivating examples for Björner's original work [21] on EL-shellability. Fix an ordering 
$a_{1}, a_{2}, \ldots, a_{k}$ of the atoms of the geometric lattice $L$. Then label each edge $x<y$ of the Hasse diagram with the smallest $i$ for which $x \vee a_{i}=y$. Note that if the atoms of the subset lattice $B_{n}$ are ordered $\{1\},\{2\}, \ldots,\{n\}$, then the geometric lattice EL-labeling is precisely the labeling given in Section 3.2.1.

\section{Exercise 3.2.14.}

(a) Show that the edge labeling for geometric lattices described above is an EL-labeling.

(b) Find an ordering of the atoms of $\Pi_{n}$ such that the induced geometric lattice EL-labeling is equivalent to the EL-labeling $\lambda_{1}$ of Section 3.2.2.

(c) Is $\lambda_{2}$ of Section 3.2.2 equivalent to a geometric lattice EL-labeling for some ordering of the atoms?

(d) Show that every semimodular lattice has an EL-labeling.

The decreasing chains of the geometric lattice EL-labeling have a very nice characterization, due to Björner [22], which is described in the language of matroid theory. A set $A$ of atoms in a geometric lattice $L$ is said to be independent if $r(\vee A)=|A|$. A set of atoms that is minimally dependent (i.e., every proper subset is independent) is called a circuit. An independent set of atoms that can be obtained from a circuit by removing its smallest element (with respect to the fixed ordering $a_{1}, a_{2}, \ldots, a_{k}$ of the atoms of $L$ ) is called a broken circuit. A maximal independent set of atoms is said to be an NBC base if contains no broken circuits. There is a natural bijection from the NBC bases of $L$ to the decreasing chains of $L$. Indeed, given any NBC base $A=\left\{a_{i_{1}}, \ldots, a_{i_{r}}\right\}$, where $1 \leq i_{1}<i_{2}<\cdots<i_{r} \leq k$, construct the maximal chain

$$
c_{A}:=\left(\hat{0}<a_{i_{r}}<a_{i_{r}} \vee a_{i_{r-1}}<\cdots<a_{i_{r}} \vee a_{i_{r-1}} \vee \cdots \vee a_{i_{1}}=\hat{1}\right) .
$$

It is not difficult to check that the label sequence of $c_{A}$ is $\left(i_{r}, i_{r-1}, \ldots, i_{1}\right)$, which is decreasing, and that the map $A \mapsto c_{A}$ is a bijection from the NBC bases of $L$ to the decreasing chains of $L$. We conclude that the set $\left\{\bar{c}_{A}: A\right.$ is an NBC base of $\left.L\right\}$ is a basis for top cohomology of $L$.

Björner [22] also constructs a basis for homology of $L$ indexed by the NBC bases, which is dual to the cohomology basis described above. Any independent set of atoms in a geometric lattice generates (by taking joins) a Boolean algebra embedded in the geometric lattice. Given any independent set of atoms $A$, let $L_{A}$ be the Boolean algebra generated by $A$ and let $\rho_{A}$ be the fundamental cycle of $\bar{L}_{A}$.

Theorem 3.2.15 (Björner [22]). Fix an ordering of the set of atoms of a geometric lattice $L$. The set

$$
\left\{\rho_{A}: A \text { is an } N B C \text { base of } L\right\}
$$

is a basis for top homology of $\bar{L}$, which is dual to the decreasing chain basis

$$
\left\{\bar{c}_{A}: A \text { is an } N B C \text { base of } L\right\}
$$

for top cohomology.

Exercise 3.2.16. Prove Theorem 3.2.15.

Exercise 3.2.17. Show that the tree splitting basis for homology of the partition lattice is an example of a Björner NBC basis.

For further reading on the homology of geometric lattices see Björner's book chapter $[\mathbf{2 6}]$. 


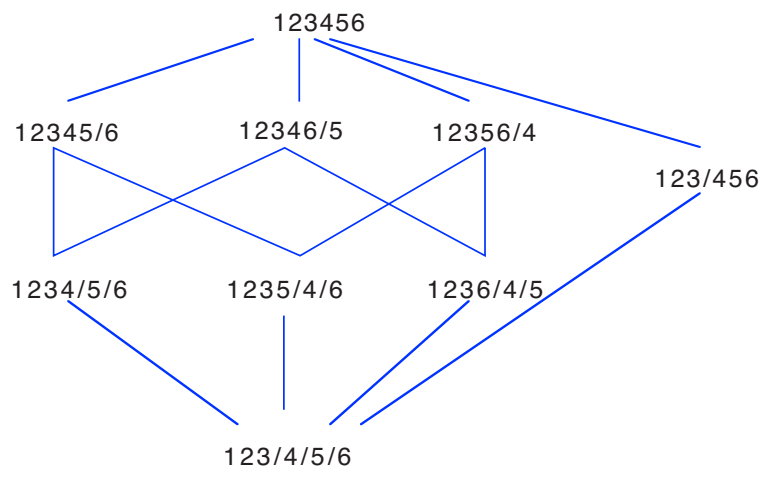

Figure 3.2.6. Interval of $\Pi_{6,3}$

\subsubsection{The $k$-equal partition lattice}

In this section we present the original example that motivated the Björner-Wachs extension of the theory of shellability from pure to nonpure complexes. This interesting example played a pivotal role in two other important developments, namely the use of poset topology in complexity theory (Björner, Lovász and Yao $[\mathbf{3 4}, \mathbf{3 3}]$ ) and the derivation of the equivariant version of the Goresky-MacPherson formula (Sundaram and Welker [184]).

The $k$-equal partition lattice $\Pi_{n, k}$ is the subposet of $\Pi_{n}$ consisting of partitions that have no blocks of size $\{2,3, \ldots, k-1\}$. This lattice is not a geometric lattice when $k>2$. It's not even pure; consider the interval of $\Pi_{6,3}$ shown in Figure 3.2.6.

The $k$-equal partition lattice arose in the early 1990's from the " $k$-equal problem" in complexity theory: Given a sequence of $n$ real numbers $x_{1}, \ldots, x_{n}$, determine whether or not some $k$ of them are equal. Björner, Lovász and Yao $[\mathbf{3 4}, \mathbf{3 3}]$ obtained the following lower bound on the complexity of the $k$-equal problem by studying the topology of $\bar{\Pi}_{n, k}$,

$$
\max \left\{n-1, n \log _{3} \frac{n}{3 k}\right\} .
$$

This lower bound differs by a factor of only 16 from the best upper bound. The $k$ equal problem translates into a subspace arrangement problem; namely determine whether or not the point $\left(x_{1}, \ldots, x_{n}\right)$ is in the complement of the real subspace arrangement $\mathcal{A}_{n, k}$, called the $k$-equal arrangement, consisting of subspaces of the form

$$
\left\{\left(x_{1}, \ldots, x_{n}\right) \in \mathbb{R}^{n}: x_{i_{1}}=x_{i_{2}}=\cdots=x_{i_{k}}\right\},
$$

where $1 \leq i_{1}<\cdots<i_{k} \leq n$. Björner and Lovász [33] show that the sum of the Betti numbers of the complement of the $k$-equal arrangement gives a lower bound for the depth of the best decision tree for the $k$-equal problem. They then use the Goresky-MacPherson formula (Theorem 1.3.8) to reduce the complexity problem to that of studying the topology of lower intervals in the intersection lattice of $\mathcal{A}_{n, k}$, which is isomorphic to $\Pi_{n, k}$. 
Björner and Welker [45] prove that all open intervals of $\Pi_{n, k}$ have the homotopy type of a wedge of spheres of varying dimensions. Since homotopy type of a wedge of spheres (of top dimension) is the main topological consequence of pure shellability, this result led Björner and Wachs to consider shellability for nonpure complexes.

We now present the nonpure EL-labeling of $\Pi_{n, k}$ obtained in [40]. First linearly order the label set $\{\overline{1}<\overline{2}<\cdots<\bar{n}<1<2<\cdots<n\}$. Now label the edge $\pi<\tau$ as follows:

$\lambda(\pi, \tau)= \begin{cases}\max B & \text { if a new block } B \text { is formed from singleton blocks } \\ a & \text { if a nonsingleton block is merged with a singleton }\{a\} \\ \max B_{1} \cup B_{2} & \text { if two nonsingletons } B_{1} \text { and } B_{2} \text { are merged. }\end{cases}$

Exercise 3.2.18. Show that $\lambda$ is an EL-labeling of $\Pi_{n, k}$.

We next describe the decreasing maximal chains. Given a hook shape Young diagram $k 1^{m}$, by the corner of the hook we mean the cell in the first row and first column, and by the leg of $T$ we mean the first column of $T$ minus the corner. We refer to a skew diagram as a $k$-broken skew hook diagram if it is the disjoint union of hooks $H_{1}, H_{2}, \ldots, H_{t}$ of the form $k 1^{m}$, where $m$ is arbitrary. The tableaux in Figure 3.2.7 are reverse standard tableaux (i.e. column and row entries are decreasing rather than increasing) of 3-broken skew hook shape. For each reverse standard tableau $T$ of $k$-broken skew hook shape, with entries in $[n]$, let $\pi_{T}$ be the partition in $\Pi_{n, k}$ whose nonsingleton blocks are $B_{1}, \ldots, B_{t}$, where $B_{i}$ is the set of entries of $T$ in the hook $H_{i}$. For the tableaux in Figure 3.2.7,

$$
\pi_{T}=15,14,3,9,8,5 / 10,7,1,2 / 13,11,4,12,6
$$

and

$$
\pi_{T^{\prime}}=15,14,3,9,8,5 / 10,7,1 / 13,11,4,12,6 / 2 .
$$

Given a reverse standard tableau $T$ of $k$-broken skew hook shape, we let $a$ be the smallest entry among all entries of $T$ that are either in the leg of a hook or in the corner of a hook that has no leg. In the former case we let $T^{\prime}$ be the standard tableau of $k$-broken skew hook shape obtained by removing $a$ from $T$. In the latter case we let $T^{\prime}$ be the standard tableau of $k$-broken skew hook shape obtained by removing the entire hook (which is a row) containing $a$. In either case, we refer to the tableau $T^{\prime}$ as the predecessor of $T$. In Figure 3.2.7, $T^{\prime}$ is the predecessor of $T$. For each reverse standard tableau $T$ of $k$-broken skew hook shape, with $n$ cells and entries in $[n]$, let $c_{T}$ be the maximal chain of $\Pi_{n, k}$ such that

- $\pi_{T} \in c_{T}$

- the upper segment $c_{T} \cap\left[\pi_{T}, \hat{1}\right]$ is the chain

$\pi_{T}=B_{1} / B_{2} / \ldots / B_{t}<B_{1} \cup B_{2} / B_{3} / \ldots / B_{t}<\cdots<B_{1} \cup \cdots \cup B_{t}=\hat{1}$

- the lower segment $c_{T} \cap\left[\hat{0}, \pi_{T}\right]$ is the chain

$$
\hat{0}=\pi_{T_{0}}<\pi_{T_{1}}<\cdots<\pi_{T_{n-t(k-1)}}=\pi_{T},
$$

where $T_{i}$ is the predecessor of $T_{i+1}$.

For the tableau $T$ of Figure 3.2.7, the maximal chain $c_{T}$ is given by 


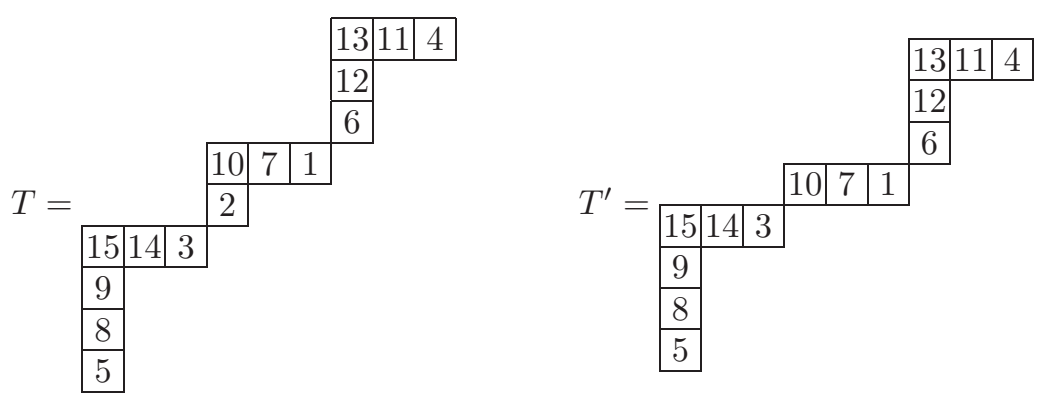

Figure 3.2.7.

$$
\begin{aligned}
\hat{1} & >15,14,3,9,8,5,10,7,1,2 / 13,11,4,12,6 \\
& >15,14,3,9,8,5 / 10,7,1,2 / 13,11,4,12,6 \\
& >15,14,3,9,8,5 / 10,7,1 / 13,11,4,12,6 \\
& >15,14,3,9,8 / 10,7,1 / 13,11,4,12,6 \\
& >15,14,3,9,8 / 10,7,1 / 13,11,4,12 \\
& >15,14,3,9 / 10,7,1 / 13,11,4,12 \\
& >15,14,3 / 10,7,1 / 13,11,4,12 \\
& >15,14,3 / 13,11,4,12 \\
& >15,14,3 / 13,11,4 \\
& >15,14,3 \\
& >0
\end{aligned}
$$

where only the nonsingleton blocks are shown.

\section{Exercise 3.2.19.}

(a) [40] Show that the set of decreasing chains with respect to $\lambda$ is the set

$\left\{c_{T}: T\right.$ is a reverse standard tableau of $k$-broken skew hook shape, with $n$ cells and $n$ in the corner of the leftmost hook\}.

(b) [40] Show that the Betti numbers are given by

$$
\tilde{\beta}_{n-3-t(k-2)}\left(\bar{\Pi}_{n, k}\right)=\sum_{\substack{j_{1}+j_{2}+\ldots+j_{t}=n \\
j_{i} \geq k}}\left(\begin{array}{c}
n-1 \\
j_{1}-1, j_{2}, \ldots, j_{t}
\end{array}\right) \prod_{i=1}^{t}\left(\begin{array}{c}
j_{i}-1 \\
k-1
\end{array}\right)
$$

where $t \geq 1$, and

$$
\tilde{\beta}_{i}\left(\bar{\Pi}_{n, k}\right)=0
$$

if $i$ is not of the form $n-3-t(k-2)$ for any $t \geq 0$.

(c) $[\mathbf{1 8 2}]$ Prove that

$$
\tilde{H}_{n-3-t(k-2)}\left(\bar{\Pi}_{n, k}\right) \downarrow_{\mathfrak{S}_{n-1}}^{\mathfrak{S}_{n}} \cong \mathfrak{S}_{n-1} \bigoplus_{D} S^{D}
$$


summed over all $k$-broken skew hook diagrams $D$ of size $n$ in which the corner of the leftmost hook is removed. This is equivalent to

$$
\tilde{H}_{n-3-t(k-2)}\left(\bar{\Pi}_{n, k}\right) \downarrow \mathfrak{S}_{\mathfrak{S}_{n-1}}^{\mathfrak{S}_{n}} \cong \bigoplus_{\substack{j_{1}+j_{2}+\ldots+j_{t}=n \\ j_{i} \geq k}} S^{1^{j^{j_{t}-k}}} \bullet S^{(k-1)} \bullet \prod_{i=1}^{t-1} S^{\left(k 1^{j_{i}-k}\right)},
$$

where $\bullet$ and $\prod$ denote induction product.

The decreasing chains given in Exercise 3.2.19(a) were also used by Sundaram and Wachs $[\mathbf{1 8 2}]$ to obtain a formula for the unrestricted representation of $\mathfrak{S}_{n}$ on $\tilde{H}_{n-3-t(k-2)}\left(\bar{\Pi}_{n, k}\right)$, involving composition product of representations. In order to transfer the representation of the symmetric group on homology of the $k$ equal partition poset to representations of the symmetric group on the homology of the complement $M_{\mathcal{A}_{n, k}}:=\mathbb{R}^{n}-\cup \mathcal{A}_{n, k}$ of the real $k$-equal arrangement $\mathcal{A}_{n, k}$ and the complement $M_{\mathcal{A}_{n, k}^{\mathbb{C}}}:=\mathbb{C}^{n}-\cup \mathcal{A}_{n, k}^{\mathbb{C}}$ of the complex $k$-equal arrangement $\mathcal{A}_{n, k}^{\mathbb{C}}$, Sundaram and Welker derived an equivariant version of the Goresky-MacPherson formula; see Theorem 5.4.2. By computing the multiplicity of the trivial representation in the homology of the complement, they obtain Betti numbers for the orbit spaces $M_{\mathcal{A}_{n, k}} / \mathfrak{S}_{n}$ and $M_{\mathcal{A}_{n, k}}^{\mathbb{C}} / \mathfrak{S}_{n}$. For instance, they recover the following result of Arnol'd [3]:

$$
\tilde{\beta}_{i}\left(M_{\mathcal{A}_{n, k}}^{\mathbb{C}} / \mathfrak{S}_{n}\right)= \begin{cases}1 & \text { if } i=2 k-3 \\ 0 & \text { otherwise. }\end{cases}
$$

The orbit space $M_{\mathcal{A}_{n, k}}^{\mathbb{C}} / \mathfrak{S}_{n}$ is homeomorphic to the space of monic polynomials of degree $n$ whose roots have multiplicity at most $k-1$.

Type $B$ and $D$ analogs of the $k$-equal arrangement and the $k$-equal partition lattice were studied by Björner and Sagan [36]. Gottlieb [82] extended this study to Dowling lattices.

\subsection{CL-shellability and Coxeter groups}

We now consider a more powerful version of lexicographic shellability called chainlexicographic shellability (CL-shellability for short). This tool was introduced by Björner and Wachs in the early 1980's in order to establish shellability of Bruhat order on a Coxeter group [37].

For a bounded poset $P$, let $\mathcal{M E}(P)$ be the set of pairs $(c, x<y)$ consisting of a maximal chain $c$ and an edge $x<\cdot y$ along that chain. A chain-edge labeling of $P$ is a map $\lambda: \mathcal{M E}(P) \rightarrow \Lambda$, where $\Lambda$ is some poset, satisfying: If two maximal chains coincide along their bottom $d$ edges, then their labels also coincide along these edges.

An example of a chain-edge labeling is given in Figure 3.3.1. Note that one of the edges has two labels. This edge receives label 3 if it is paired with the leftmost maximal chain and receives label 1 if it is paired with the other maximal chain that contains the edge.

Just as for edge labelings, we need to restrict chain-edge labelings $\lambda: \mathcal{M E}(P) \rightarrow$ $\Lambda$ to intervals $[x, y]$. The complication is that since there can be more than one way to label an edge depending upon which maximal chain it is paired with, there can be more than one way to restrict $\lambda$ to $\mathcal{M E}([x, y])$. It follows from the definition of 


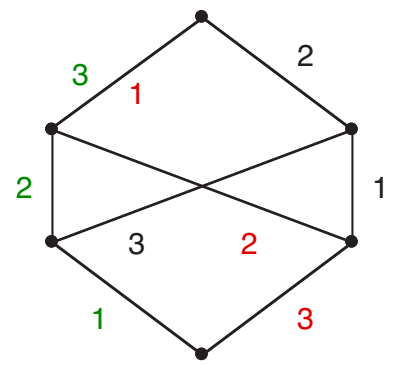

Figure 3.3.1. chain edge labeling

chain-edge labeling that each maximal chain $r$ of $[\hat{0}, x]$ determines a unique restriction of $\lambda$ to $\mathcal{M E}([x, y])$. This enables one to talk about increasing and decreasing maximal chains and lexicographic order of maximal chains in the rooted interval $[x, y]_{r}$.

Definition 3.3.1. Let $P$ be a bounded poset. A chain-lexicographic labeling (CLlabeling, for short) of $P$ is a chain-edge labeling such that in each closed rooted interval $[x, y]_{r}$ of $P$, there is a unique strictly increasing maximal chain, which lexicographically precedes all other maximal chains of $[x, y]_{r}$. A poset that admits a CL-labeling is said to be CL-shellable.

The chain-edge labeling of Figure 3.3.1 is a CL-labeling. The unique increasing maximal chain of the poset is the leftmost maximal chain.

It is easy to see that EL-shellability implies CL-shellability. All the consequences of EL-shellability discussed in Section 3.2 are also consequences of the more general CL-shellability. It is unknown whether CL-shellability and EL-shellability are equivalent notions.

We now present the original example that motivated this more technical version of lexicographic shellability.

Definition 3.3.2. A Coxeter system $(W, S)$ consists of a a group $W$ together with a set of generators $S$ such that the following relations form a presentation of $W$ :

- $s^{2}=e$, for all $s \in S$

- $(s t)^{m_{s, t}}=e$, where $m_{s, t} \geq 2$, for certain $s \neq t \in S$.

The group $W$ is said to be a Coxeter group.

Finite Coxeter groups can be characterized as finite reflection groups, i.e, finite groups generated by linear reflections in Euclidean space. Coxeter groups are an important class of groups, which have fascinating connections to many areas of mathematics, including combinatorics; see the chapter by Fomin and Reading in this volume $[\mathbf{7 1}]$ and the recent book of Björner and Brenti [30]. The finite irreducible Coxeter groups have been completely classified. There are four infinite families and six exceptional irreducible Coxeter groups. The most basic family consists of the type A Coxeter groups, which are the symmetric groups $\mathfrak{S}_{n}$ with the adjacent transpositions $(i, i+1), i=1, \ldots, n-1$, forming the generating set. This is the reflection group of the (type A) braid arrangement. It is also the group of symmetries of the $n$-simplex. The hyperoctahedral groups $\mathfrak{S}_{n}\left[\mathbb{Z}_{2}\right]$ with generators given by signed adjacent transpositions $(1,-1)$ and $(i, i+1), i=1, \ldots, n-1$, form 
the type B family. This is the reflection group of the type B braid arrangement. It is also the symmetry group of the $n$-cube and the $n$-cross-polytope.

Let $(W, S)$ be a Coxeter system. Every $\sigma \in W$ can be expressed as a product,

$$
\sigma=s_{1} \ldots s_{k},
$$

where $s_{i} \in S$. The word $s_{1} \ldots s_{k}$ is said to be a reduced expression for $\sigma$ if its length $k$ is minimum among all words whose product is $\sigma$. The length $l(\sigma)$ of $\sigma$ is defined to be the length of a reduced expression for $\sigma$.

Bruhat order on $W$ is a partial order relation on $W$ that is defined via the covering relation, $\sigma<\tau$ if

- $\tau=t \sigma$, for some $t \in T:=\left\{\alpha s \alpha^{-1}: s \in S, \alpha \in W\right\}$

- $l(\tau)=l(\sigma)+1$.

For any subset $J$ of the generating set $S$, there is an induced subposet of Bruhat order on $W$, called the quotient by $J$, defined as follows

$$
W^{J}:=\{\sigma \in W: s \sigma>\sigma \text { for all } s \in J\} .
$$

Note $W=W^{\emptyset}$.

Bruhat order describes the inclusion relationships of the Schubert subvarieties of a flag manifold. It was conjectured by de Concini and Stanley in the late 1970's that any open interval of of Bruhat order on a quotient of a Coxeter group is homeomorphic to a $d$-sphere or a $d$-ball, where $d$ is the length of the interval. This result was needed by de Concini and Lakshmibai [57] in their work on CohenMacaulayness of homogeneous coordinate rings of certain generalized Schubert varieties. In an attempt to prove the conjecture by establishing EL-shellability, Björner and Wachs instead came up with the notion of CL-shellability and constructed a CL-labeling of the dual Bruhat poset (call this a dual CL-labeling). This labeling relied on the following well-known characterization of Bruhat order.

Theorem 3.3.3 (Subword characterization of Bruhat order). Let $(W, S)$ be a Coxeter system. Then $\sigma<\tau$ in Bruhat order on $W$ if and only if for any reduced expression $w$ for $\tau$ there is a reduced expression for $\sigma$ that is a subword of $w$.

We describe the dual CL-labeling of intervals $[\sigma, \tau]$ of $W^{J}$ (this works for infinite $W$ as well as finite $W$ ). Fix a reduced expression $w$ for $\tau$. It follows easily from the subword characterization that as we travel down a maximal chain, we delete (unique) letters of $w$ one at a time until we reach a reduced word for $\sigma$. Label the edges of each maximal chain from top down with the position of the letter in $w$ that is crossed out. This is illustrated in Figure 3.3.2 on the full interval $[e, 321]$ of Bruhat order on $\mathfrak{S}_{3}$, where $s_{i}$ denotes the adjacent transposition $(i, i+1)$.

Theorem 3.3.4 (Björner and Wachs [37]). The dual chain-edge labeling described above is a dual CL-labeling of closed intervals of Bruhat order on $W^{J}$. The number of decreasing chains (from top to bottom) is 1 if $J=\emptyset$ and is at most 1 otherwise.

It follows from Theorem 3.3.4 that every open interval $(\sigma, \tau)$ of $W^{J}$ has the homotopy type of a $(l(\tau)-l(\sigma)-2)$-sphere or is contractible. A stronger topological consequence can be obtained by using the Danaraj and Klee result (Theorem 3.1.11). Indeed, it can be shown that every closed interval of length 2 in $W$ has exactly 4 elements and that every closed interval of length 2 in $W^{J}$ has at most 4 elements. 


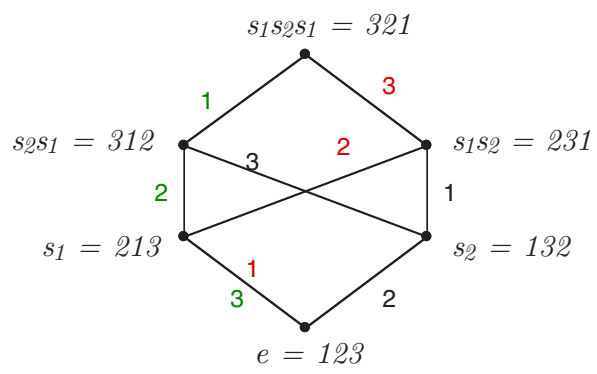

Figure 3.3.2. Dual CL-labeling of $\mathfrak{S}_{3}$

Corollary 3.3.5. Every open interval $(\sigma, \tau)$ of Bruhat order on a quotient $W^{J}$ of a Coxeter group $W$ is homeomorphic to a $(l(\tau)-l(\sigma)-2)$-sphere or a $(l(\tau)-l(\sigma)-2)$ ball. If $J=\emptyset$ then $(\sigma, \tau)$ is homeomorphic to a $(l(\tau)-l(\sigma)-2)$-sphere.

Besides for quotients, there are other interesting classes of induced subposets of Bruhat order such as descent classes, studied by Björner and Wachs [39], and the subposet of involutions, which arose in algebraic geometry work of Richardson and Springer [143]. There is a natural notion of descent set for general Coxeter groups which generalizes the descent set of a permutation. A descent class of a Coxeter system $(W, S)$ is the set of all elements whose descent set is some fixed subset of $S$. Björner and Wachs used the chain-edge labeling described above to show that every finite interval of any descent class of any Coxeter group is dual CL-shellable. This chain-edge labeling does not work for the subposet of involutions because the maximal chains of the subposet are not maximal in the full poset. Recently Incitti found an EL-labeling that does work for the classical Weyl groups (i.e., the type A, B or D Coxeter groups) and conjectured that his result extends to all intervals of all (finite or infinite) Coxeter groups. More recently, Hultman [98] proved the main consequence of this conjecture, namely that all intervals of Bruhat order on the set of involutions of a Coxeter group are homeomorphic to spheres.

An alternative way to label the edges of Hasse diagram of $W^{J}$ is by labeling the edge $\sigma \lessdot \tau$ with the element $t \in T$ such that $\tau=t \sigma$. By imposing a certain linear order on $T$, Edelman [67] showed that this edge labeling is an EL-labeling for the symmetric group. Proctor [132] did the same for the classical Weyl groups. Several years after the introduction of CL-shellability, Dyer [65] found a way to linearly order $T$ so that the edge labeling by elements of $T$ is an EL-labeling for all Coxeter groups and all quotients. Dyer's linear order (called reflection order) was recently used by Williams [210] to obtain an EL-labeling of the poset of cells in a certain cell decomposition of Rietsch [144] of the totally nonnegative part of an arbitrary flag variety (for any reductive linear algebraic group). This and the fact that the poset is thin (cf., Theorem 3.1.12) led Williams to conjecture that Rietsch's cell decomposition is a regular cell decomposition of a ball (if true this would improve a result of Lusztig [122] asserting that the totally nonnegative part of the flag variety is contractible). 
There are two other natural partial order relations on a Coxeter group $(W, S)$ that are important and interesting. By replacing $T$ by $S$ in the definition of Bruhat order, one gets the definition of weak order. By replacing ordinary length $l$ by absolute length $a l$ in the definition of Bruhat order, where $a l(\sigma)$ is the length of the shortest factorization of $\sigma$ in elements of $T$, one gets the definition of absolute length order.

The Hasse diagram of weak order is the same as the Cayley graph of the group $W$ with respect to $S$, directed away from the identity. It is pure and bounded with the same rank function as Bruhat order, ordinary length $l$. Its topology was first studied by Björner [24] who showed that although it is not lexicographically shellable, it has the homotopy type of a sphere.

The Hasse diagram of absolute length order $W^{a l}$ is the same as the Cayley graph of the group $W$ with respect to $T$, directed away from the identity. It is pure with rank function, absolute length al; its bottom element is $e$, but it has no unique top element. In the symmetric group $\mathfrak{S}_{n}$, the maximal elements are the $n$-cycles. Interest in the absolute length order is a fairly recent development, which arose in work on the braid group [48], [49], [17]. It was shown by Brady [48] that if $W$ is the symmetric group $\mathfrak{S}_{n}$, then every interval $[e, c]$ of $W^{a l}$, where $c$ in an $n$-cycle, is isomorphic to the noncrossing partition lattice $N C_{n}$ discussed in Section 3.2.2. This observation (and connections to finite type Artin groups) led Bessis [17] and Brady and Watt [49] to define the noncrossing partition poset for any finite Coxeter group to be the interval $[e, c]$ of $W^{a l}$, where $c$ is a Coxeter element of $(W, S)$. A Coxeter element is a product of all the elements of $S$ in some order. In $\mathfrak{S}_{n}$ the Coxeter elements are the $n$-cycles. Reiner's type B noncrossing partition lattice as well as the classical (type A) noncrossing partition lattice are recovered from this definition.

Exercise 3.3.6 (Brady [48]). Show that the interval $[e, c]$, where $c$ is an $n$-cycle, in absolute length order of the symmetric group is isomorphic to the noncrossing partition lattice $\mathrm{NC}_{n}$.

Although the topology of the interval $(e, c)$, where $c$ is a Coxeter element, is known to have the homotopy type of a wedge of spheres via the AthanasiadisBrady-Watt proof of EL-shellability [6], little is known about the topology of the full absolute length poset, $W^{a l}$, even for the symmetric group.

Problem 3.3.7 (Reiner [2, Problem 3.1]). What can be said about the topology of $W^{a l}-\{e\}$, where $W$ is an arbitrary finite Coxeter group? Is $W^{a l}$ lexicographically shellable, for types $A$ and $B$ ? It is known that for type $D$, the poset $W^{a l}$ is not shellable.

In the next exercise we see other examples of posets that admit CL-labelings.

Exercise 3.3.8. Let $\mathcal{W}_{n}$ be the poset of finite words over alphabet $[n]$, ordered by the subword relation. We have $34<23244$ in $\mathcal{W}_{n}$. Let $\mathcal{N}_{n}$ be the induced subposet of normal words, where a word is said to be normal if no two adjacent letters are equal. For example, 2324 is normal while 23244 is not.

(a) (Björner and Wachs [38]) Find a dual CL-labeling of each interval $[u, v]$ in $\mathcal{N}_{n}$, and show that $(u, v)$ is homeomorphic to an $(l(v)-l(u)-2)$-sphere.

(b) (Björner [25]) Find a dual CL-labeling of each interval $[u, v]$ in $\mathcal{W}_{n}$. 
(c) (Björner $[\mathbf{2 5}, \mathbf{2 7}])$ Given a word $v=a_{1} \ldots a_{n}$ in $\mathcal{W}_{n}$, define its repetition set $R(v)=\left\{i: a_{i}=a_{i-1}\right\}$. A normal embedding of a word $u$ in $v=$ $a_{1} \ldots a_{n}$ is a sequence $1 \leq i_{i}<\ldots<i_{k} \leq n$ such that $u=a_{i_{1}} \ldots a_{i_{k}}$ and $R(v) \subseteq\left\{i_{1}, \ldots, i_{k}\right\}$. Show that the interval $(u, v)$ in $\mathcal{W}_{n}$ is homotopy equivalent to a wedge of $n_{u, v}$ spheres of dimension $l(v)-l(u)-2$, where $n_{u, v}$ is the number of normal embeddings of $u$ in $v$.

In the next lecture, a formulation of CL-shellability, called recursive atom ordering, which does not involve edge labelings, will be presented. This formulation has proved to be quite useful in many applications, indeed, even more useful than the original chain labeling version. A more general form of lexicographic shellability which does involve edge labelings was introduced by Kozlov [110]. A generalization of lexicographic shellability to balanced complexes was formulated by Hersh [93] and was further studied by Hultman [97]. A relative version of lexicographic shellability was introduced by Stanley [170]. A Morse theory version of lexicographical shellability, which is even more general than Kozlov's version was formulated by Babson and Hersh [9].

\subsection{Rank selection}

Edge labelings of posets were first introduced by Stanley $[\mathbf{1 6 2}, \mathbf{1 6 3}, \mathbf{1 6 4}]$ for the purpose of studying the Möbius function of rank-selected subposets of certain pure lattices. If one drops the requirement that the unique increasing maximal chain be lexicographically first in the definition of EL-shellability then one has the kind of labeling that Stanley considered. Such a labeling was called an R-labeling (we now call it an ER-labling and we call the chain-edge version a CR-labeling). Here we discuss how CL-labelings are used to determine homotopy type and homology of rank-selected subposets of pure CL-shellable posets.

Let $P$ be a bounded poset. For $x \in P$, define the $r a n k$,

$$
r(x):=l([\hat{0}, x]) \text {. }
$$

For $R \subseteq[l(P)-1]$, define the rank-selected subposet

$$
P_{R}:=\{x \in P: r(x) \in R\} .
$$

Given a CL-labeling of a bounded poset $P$, the descent set of a maximal chain $c:=\left(\hat{0}=x_{0}<x_{1}<x_{2}<\ldots<x_{t}<x_{t+1}=\hat{1}\right)$ of $P$ is defined to be

$$
\operatorname{des}(c):=\left\{i \in[t]: \lambda\left(c, x_{i-1}<x_{i}\right) \geq \lambda\left(c, x_{i}<x_{i+1}\right)\right\} .
$$

Theorem 3.4.1 (Björner and Wachs [38]). Suppose $P$ is pure and $C L$-shellable. Let $R \subseteq[l(P)-1]$. Then $\hat{P}_{R}$ is also $C L$-shellable, and hence $P_{R}$ has the homotopy type of a wedge of $(|R|-1)$-spheres. The number of spheres is the number of maximal chains of $P$ with descent set $R$. Moreover, the set

$$
\left\{c_{R}: c \in \mathcal{M}(P) \& \operatorname{des}(c)=R\right\}
$$

forms a basis for $\tilde{H}^{|R|-1}\left(P_{R} ; \mathbb{Z}\right)$.

Remark 3.4.2. Theorem 3.4.1 holds for nonpure posets only when the rank set $R$ is rather special, see [41]. It was shown by Björner [21] that pure shellability is preserved by rank selection. It is not known, however, whether pure EL-shellability is preserved by rank-selection. Stanley $[\mathbf{1 6 3}, \mathbf{1 6 4}]$ proved the precursor to Theorem 3.4.1 that for pure posets $P$ that admit ER-labelings, the rank selected Möbius 
invariant $\mu\left(\hat{P}_{R}\right)$ is equal to $(-1)^{|R|-1}$ times the number of maximal chains of $P$ with descent set $R$.

Example 3.4.3. The rank-selected Boolean algebra $\left(B_{n}\right)_{R}$. Consider the ELlabeling of $B_{n}$ given in Example 3.2.1. Since the label sequences of the maximal chains are the permutations in $\mathfrak{S}_{n}$, the number of maximal chains with descent set $R \subseteq[n-1]$ is the number of permutations with descent set $R$. So $\left(B_{n}\right)_{R}$ has the homotopy type of a wedge of $d_{n, R}$ spheres of dimension $|R|-1$, where $d_{n, R}$ is the number of permutations in $\mathfrak{S}_{n}$ with descent set $R$. (Note that the truncated Boolean algebra given in Section 3.2.1 is an example of a rank-selected Boolean algebra.) We have the following equivariant homology version of the homotopy result.

Theorem 3.4.4 (Solomon [161]). Let $R \subseteq[n-1]$ and let $H$ be the skew hook with $n$ cells and descent set $R$ (the definition of descent set of a skew hook was given in Section 2.2). Then

$$
\tilde{H}_{|R|-1}\left(\left(B_{n}\right)_{R}\right) \cong_{\mathfrak{S}_{n}} S^{H} .
$$

Exercise 3.4.5. Prove Theorem 3.4.4 by using a map from the set of tableaux of shape $\mathrm{H}$ to the set of maximal chains of $\left(B_{n}\right)_{R}$ thereby generalizing the proof of Theorem 3.2.7.

We consider a type B-analog and a q-analog of Example 3.4.3 in the next two exercises.

Exercise 3.4.6. The rank-selected lattice of faces of the $n$-cross-polytope $\left(C_{n}\right)_{R}$. Recall form Example 2.3.3 that we can identify the $(k-1)$-faces of $C_{n}$ with $k$-subsets $T$ of $[n] \cup\{-i: i \in[n]\}$ such that $\{i,-i\} \nsubseteq T$ for all $i$. Note that the maximal chains of $C_{n}$ correspond bijectively to elements of the hyperoctahedral group $\mathfrak{S}_{n}\left[\mathbb{Z}_{2}\right]$, i.e. the signed permutations. As was mentioned earlier, there is a natural notion of descent set for general Coxeter groups which generalizes the descent set of a permutation. For the hyperoctahedral group, descent can be characterized as follows: $i \in\{0,1, \ldots, n-1\}$ is a descent of a signed permutation $\sigma \in \mathfrak{S}_{n}\left[\mathbb{Z}_{n}\right]$ if either

(a) $\sigma(i)>\sigma(i+1)$ (viewed as elements of $\mathbb{Z}$ ) or

(b) $i=0$ and $\sigma(1)<0$.

Find an $E L$-labeling of $C_{n}$ such that for all $R \subseteq[n]$, the number of maximal chains with descent set $R$ equals $\bar{d}_{n, R}$, the number of signed permutations with descent set $R$. Consequently, $\left(C_{n}\right)_{R}$ has the homotopy type of a wedge of $\bar{d}_{n, R}$ spheres of dimension $|R|-1$.

Exercise 3.4.7 (Simion $[\mathbf{1 5 8}]$ ). The rank-selected subspace lattice $\left(B_{n}(q)\right)_{R}$. Find an EL-labeling $\lambda$ of $B_{n}(q)$ with label set $[n]$ such that

- for each maximal chain $c$, the label sequence $\lambda(c)$ is a permutation in $\mathfrak{S}_{n}$,

- for each permutation $\sigma \in \mathfrak{S}_{n}$, the number of maximal chains $c$ such that $\lambda(c)=\sigma$ is $q^{\operatorname{inv}(\sigma)}$, where $\operatorname{inv}(\sigma)$ is the number of inversions of $\sigma$.

Consequently, for all $R \subseteq[n-1]$, the rank-selected subspace lattice $\left(B_{n}(q)\right)_{R}$ has the homotopy type of a wedge of $d_{n, R}(q)$ spheres of dimension $|R|-1$, where

$$
d_{n, R}(q)=\sum_{\substack{\sigma \in \mathfrak{S}_{n} \\ \operatorname{des}(\sigma)=R}} q^{\operatorname{inv}(\sigma)} .
$$


There are also interesting results on $\mathfrak{S}_{n}$-equivariant rank-selected homology of the partition lattice with ties to permutation enumeration due to Sundaram $[\mathbf{1 7 5}, \mathbf{1 7 7}]$. See also the related recent work of Hanlon and Hersh [88] on rankselected partition lattices [88]

Exercise 3.4.8. Let $L$ be a geometric lattice with the EL-labeling given in Section 3.2.3. Also let $R \subseteq[l(L)-1]$. Show that there is a basis for top homology of $L_{R}$ consisting of fundamental cycles of subposets of $L_{R}$ that are all isomorphic to the join $\bar{B}_{s_{1}} * \bar{B}_{s_{2}} * \cdots * \bar{B}_{s_{k}}$, where $\left\{s_{1}, s_{1}+s_{2}, \ldots, s_{1}+s_{2}+\cdots+s_{k}\right\}=[l(L)]-R$.

Stanley [167] gives a type B analog of Theorem 3.4.4 for the lattice of Exercise 3.4.6 and a $q$-analog of Theorem 3.4.4 for the lattice of Exercise 3.4.7. These results involve the representation theories of the hyperoctahedral group and the general linear group, which are analogous to that of the symmetric group. He proves Theorem 3.4.4 and the analogous results by invoking the following general theorem.

Theorem 3.4.9 ([167]). Let $P$ be a bounded pure shellable $G$-poset of length $l$. If $R \subseteq[l-1]$ then

$$
\tilde{H}_{|R|-1}\left(P_{R}\right) \cong_{G} \bigoplus_{T \subseteq R}(-1)^{|R-T|} C_{|T|-1}\left(P_{T}\right) .
$$

Equivalently,

$$
C_{|R|-1}\left(P_{R}\right) \cong_{G} \bigoplus_{T \subseteq R} \tilde{H}_{|T|-1}\left(P_{T}\right)
$$

In [167] Theorem 3.4.9 is proved for a more general class of posets called CohenMacaulay posets. These posets are discussed in the next section. Equation (3.4.1) is a consequence of the Hopf trace formula and the fact that pure shellability, or more generally Cohen-Macaulayness, is preserved by rank-selection. Equation (3.4.2) follows by the principle of inclusion-exclusion or Möbius inversion on the subset lattice.

Note that Theorems 3.4.4 and 3.4.9 imply the fact that the regular representation of $\mathfrak{S}_{n}$ decomposes into a direct sum of Foulkes modules, cf., Exercise 2.2.8.

Exercise 3.4.10. Prove Theorem 3.4.4 by using Theorem 3.4.9.

As was mentioned in the introduction to this lecture, shellability theory is intimately connected with the enumeration of faces of polyhedral complexes, a central topic in geometric combinatorics; see the books of Stanley [168] and Ziegler [218]. The descent set of a maximal chain is a specialization of a more general concept in shellability theory known as the restriction of a facet, which is just the smallest new face that is added to the complex when the facet is added. When facets of pure shellable simplicial complexes are enumerated according to the size of their restriction, an important combinatorial invariant of the simplicial complex, known as the $h$-vector, is computed. For any $(d-1)$-dimensional simplicial complex $\Delta$ (shellable or not), the $h$-vector $\left(h_{0}(\Delta), h_{1}(\Delta), \ldots, h_{d}(\Delta)\right)$ and the $f$-vector $\left(f_{-1}(\Delta), f_{0}(\Delta), \ldots, f_{d-1}(\Delta)\right)$ determine each other,

$$
\sum_{i=0}^{d} h_{i} x^{d-i}=\sum_{i=0}^{d} f_{i-1}(x-1)^{d-i}
$$


but the $h$-vector is usually more convenient for expressing relations such as the upper bound conjecture, a symmetry relation known as the Dehn-Sommerville equations, the celebrated Billera-Lee [19] and Stanley [166] characterization of the fvector of a simplicial polytope, and the conjectured extension to convex polytopes and homology spheres. Although shellability has played an important role in the study of $f$-vectors and $h$-vectors, much fancier tools from commutative algebra and algebraic geometry have come into play. See the books of Stanley [168] and Ziegler [218] for basic treatments of this material, the survey article of Stanley [174] for important recent developments, and the paper of Swartz [185] for even more recent developments.

Refinements of the $f$-vector and $h$-vector called the flag $f$-vector and the flag $h$-vector, respectively, (defined for all pure posets and the more general balanced complexes) have been extensively studied, beginning with the Bayer-Billera [15] analog of the Dehn-Sommerville equations; see [168] for further information. For pure lexicographically shellable posets the entries of the flag $h$-vector have a simple combinatorial interpretation as the number of maximal chains with fixed descent set.

For nonpure complexes, two-parameter generalizations of the f-vector and hvector are defined in [40]. The f-triangle and h-triangle also determine each other. The f-triangle counts faces of a simplicial complex according to the maximum size of a facet containing the face and the size of the face. For shellable complexes, it is shown in [40] that the $h$-triangle counts facets according to the size of the restriction set and the size of the facet. Duval [64] shows that the entries of the $h$-triangle are nonnegative for a more general class of complexes than the shellable complexes; namely the sequentially Cohen-Macaulay complexes, which are discussed in the next lecture. It is pointed out by Herzog, Reiner, and Welker [95] that the htriangle of a sequentially Cohen-Macaulay complex $\Delta$ encodes the multigraded Betti numbers appearing in the minimal free resolution of the Stanley-Reisner ideal of the Alexander dual of $\Delta$. Stanley-Reisner rings and ideals are discussed briefly in the next lecture and Alexander duality is discussed in the last lecture.

Descent sets and the more general restriction sets also play an important role in direct sum decompositions of Stanley-Reisner rings of shellable complexes; due to Kind and Kleinschmidt [107], Garsia [77], and Björner and Wachs [41, Section $12]$. 



\section{LECTURE 4 Recursive techniques}

The recursive definition of the Möbius function of a poset provides a recursive technique for computing the reduced Euler characteristic of the order complex of a poset. More refined recursive techniques for computing the homology of a poset are discussed in this lecture. A general class of posets to which these techniques can be applied, the Cohen-Macaulay posets or more generally the sequentially CohenMacaulay posets, are discussed in Section 4.1. A recursive formulation of CLshellability is presented in Section 4.2. In Section 4.3 the recursive techniques for computing Betti numbers are demonstrated on various examples, and in Section 4.4 equivariant versions of these techniques are also demonstrated. In Section 4.5 bases and generating sets for homology and cohomology of some of these examples are presented together with their use in computing $\mathfrak{S}_{n}$-equivariant homology.

\subsection{Cohen-Macaulay complexes}

Recall that the link of a face $F$ of a simplicial complex $\Delta$ is the subcomplex

$$
\mathrm{lk}_{\Delta} F:=\{G \in \Delta: G \cup F \in \Delta, G \cap F=\emptyset\} .
$$

Definition 4.1.1. A simplicial complex $\Delta$ is said to be Cohen-Macaulay over $\mathbf{k}$ if

$$
\tilde{H}_{i}\left(\mathrm{lk}_{\Delta} F ; \mathbf{k}\right)=0,
$$

for all $F \in \Delta$ and $i<\operatorname{dimlk}_{\Delta} F$.

The following result shows that Cohen-Macaulayness is a topological property.

Theorem 4.1.2 (Munkres [128]). The simplicial complex $\Delta$ is Cohen-Macaulay over $\mathbf{k}$ if and only if for all $p \in\|\Delta\|$ and all $i<\operatorname{dim} \Delta$,

$$
\tilde{H}_{i}(\|\Delta\| ; \mathbf{k})=H_{i}(\|\Delta\|,\|\Delta\|-p ; \mathbf{k})=0,
$$

where the homology is reduced singular homology and relative singular homology, respectively.

The Cohen-Macaulay property has its origins in commutative algebra, in the theory of Cohen-Macaulay rings. Associated with every simplicial complex $\Delta$ on vertex set $[n]$ is a ring $\mathbf{k}[\Delta]$ called the Stanley-Reisner ring of the simplicial complex, which is defined to be the quotient of the polynomial ring $\mathbf{k}\left[x_{1}, \ldots, x_{n}\right]$ by the Stanley-Reisner ideal $I_{\Delta}$ generated by monomials of the form $x_{i_{1}} x_{i_{2}} \ldots x_{i_{j}}$ where 
$\left\{i_{1}, i_{2}, \ldots, i_{j}\right\} \notin \Delta$. The Stanley-Reisner construction is a two-way bridge used to obtain topological and enumerative properties of the simplicial complex from properties of the ring and vice versa. A simplicial complex is Cohen-Macaulay if and only if its Stanley-Reisner ring is a Cohen-Macaulay ring (see [168] for the definition of Cohen-Macaulay ring). The equivalence of the characterization given in Definition 4.1.1 and the ring theoretic characterization is due to Reisner [142].

It follows from Theorem 4.1.2 that any triangulation of a $d$-sphere is CohenMacaulay. The ring theoretic consequence of this fact played an essential role in Stanley's celebrated proof of the Upper Bound Conjecture for spheres. For $n>d$, define the cyclic polytope $C(n, d)$ to be the convex hull of any $n$ distinct points on the moment curve $\left\{\left(t, t^{2}, \ldots, t^{d}\right) \in \mathbb{R}^{d}: t \in \mathbb{R}\right\}$ (the face poset of the polytope is independent of the choice of points). The boundary complex of the cyclic polytope is a simplicial complex. The upper bound conjecture for spheres asserts that the boundary complex of the cyclic polytope achieves the maximum number of faces of each dimension, over all simplicial complexes on $n$ vertices that triangulate a $d$-sphere. See [168] for Stanley's proof of this conjecture and other very important uses of the Stanley-Reisner ring and commutative algebra in the enumeration of faces of simplicial complexes.

The Stanley-Reisner bridge can also be crossed in the opposite direction obtaining ring theoretic information from the topology and combinatorics of the simplicial complex, as exemplified by the commutative algebra results mentioned at the end of the last lecture. Another example is a fundamental result of Eagon and Reiner [66] which states that a square free monomial ideal has a linear resolution if and only if it is the Stanley-Reisner ideal $I_{\Delta}$ of a simplicial complex $\Delta$ whose Alexander dual is Cohen-Macaulay. We will not define linear resolution, but Alexander duality is discussed in Section 5.1. A nonpure generalization of this result involving sequential Cohen-Macaulayness (discussed below) appears in papers of Herzog and Hibi [94] and Herzog, Reiner and Welker [95]. For further reading on the extensive connections between simplicial topology and commutative algebra, see Stanley's classic book [168] and the recent book of Miller and Sturmfels [127].

Exercise 4.1.3 (Walker [204]). Show that if $\mathrm{lk}_{\Delta} F$ is empty, 0-dimensional, or connected for all $F \in \Delta$, then $\Delta$ is pure. Consequently, Cohen-Macaulay simplicial complexes are pure.

The main tool for establishing Cohen-Macaulayness is shellability. Indeed, it follows from Theorem 3.1.5 and Corollary 3.1.4 that pure shellability implies the Cohen-Macaulay property over any k. From Exercise 4.1.3 we see that this implication does not hold for nonpure shellability. In order to extend the implication to the nonpure setting, Stanley [168] introduced nonpure versions of Cohen-Macaulay for complexes and rings, called sequentially Cohen-Macaulay, and showed that all shellable complexes are sequentially Cohen-Macaulay. Duval [64] and Wachs [199] found similar simpler characterizations of Stanley's sequential Cohen-Macaulayness for simplicial complexes. Here we take Wachs' characterization as the definition.

Definition 4.1.4. Let $\Delta$ be a simplicial complex. For each $m=1,2, \ldots, \operatorname{dim}(\Delta)$, let $\Delta^{\langle m\rangle}$ be the subcomplex of $\Delta$ generated by facets of dimension at least $m$. The complex $\Delta$ is said to be sequentially acyclic over $\mathbf{k}$ if $\tilde{H}_{i}\left(\Delta^{\langle m\rangle} ; \mathbf{k}\right)=0$ for all $i<m$. We say that $\Delta$ is sequentially Cohen-Macaulay over $\mathbf{k}$ if $\operatorname{lk}_{\Delta} F$ is sequentially acyclic over $\mathbf{k}$ for all $F \in \Delta$. 
Exercise 4.1.5. The pure $m$-skeleton $\Delta^{[m]}$ of a simplicial complex $\Delta$ is defined to be the subcomplex generated by all faces of dimension $m$. Show that the formulation of sequentially Cohen-Macaulay complex given in Definition 4.1.4 is equivalent to the following formulation of Duval [64]: $\Delta$ is sequentially Cohen-Macaulay if and only if $\Delta^{[m]}$ is Cohen-Macaulay for all $m=1,2, \ldots, \operatorname{dim}(\Delta)$.

It is clear that pure sequential Cohen-Macaulayness is the same thing as CohenMacaulayness. The following generalization of Theorem 4.1.2 shows that sequential Cohen-Macaulayness is a topological property, just as Cohen-Macaulayness is. Given a nonnegative integer $m$ and a topological space $X$, define $X^{\langle m\rangle}$ to be the topological closure of the set

$\{p \in X: p$ has a neighborhood homeomorphic to an open $d$-ball where $d \geq m\}$.

Theorem 4.1.6. The simplicial complex $\Delta$ is sequentially Cohen-Macaulay over $\mathbf{k}$ if and only if for all $i<m$ and $p \in\|\Delta\|^{\langle m\rangle}$,

$$
\tilde{H}_{i}\left(\|\Delta\|^{\langle m\rangle} ; \mathbf{k}\right)=H_{i}\left(\|\Delta\|^{\langle m\rangle},\|\Delta\|^{\langle m\rangle}-p ; \mathbf{k}\right)=0 .
$$

Exercise 4.1.7. Munkres proof of Theorem 4.1.2 is based on the following lemma: For any face $F$ of a simplicial complex $\Delta$, any point $p$ in the interior of $F$, and any integer $i$,

$$
\tilde{H}_{i}(\mathrm{lk} F ; \mathbf{k}) \cong H_{i+\operatorname{dim} F+1}(\|\Delta\|,\|\Delta\|-p ; \mathbf{k}) .
$$

Use this lemma to prove Theorem 4.1.6.

Exercise 4.1.8 (Wachs [199]). Show that if $\Delta$ is sequentially acyclic then $\tilde{H}_{i}(\Delta ; \mathbb{Z})$ is free for all $i$ and vanishes whenever there is no facet of dimension $i$.

Theorem 4.1.9 (Stanley [168]). Every shellable simplicial complex is sequentially Cohen-Macaulay over $\mathbf{k}$ for all $\mathbf{k}$.

Exercise 4.1.10. Prove Theorem 4.1.9 by first showing that if $\Delta$ is shellable then there is a shelling order of the facets of $\Delta$ in which the dimensions of the facets weakly decrease (see Björner and Wachs [40]).

We say that a poset is (sequentially) Cohen-Macaulay if its order complex is (sequentially) Cohen-Macaulay. Early work on Cohen-Macaulay posets can be found in the paper of Baclawski [12] and in the survey article of Björner, Garsia, and Stanley $[\mathbf{3 2}]$. We have the following nice characterization of sequentially CohenMacaulay posets, which, in the pure case, is well-known and follows easily from Definition 4.1.1.

Theorem 4.1.11 (Björner, Wachs, and Welker [44]). A poset $P$ is sequentially Cohen-Macaulay if and only if every open interval of $\hat{P}$ is sequentially acyclic.

One direction of the proof follows immediately from the fact that open intervals are links of chains. The other is a consequence of the fact that joins of sequentially acyclic simplicial complexes are sequentially acyclic, which is proved in [44] by using a fiber theorem of Quillen. Fiber theorems are discussed in Lecture 5. It is also shown in [44] that other poset operations such as product preserve sequential Cohen-Macaulayness, a fact that had been known for some time in the pure case [205]; see Exercise 5.1.6.

To compute the unique nonvanishing Betti number of a Cohen-Macaulay poset (or any poset in which homology is concentrated in a single dimension), one needs 
only to compute its Möbius invariant. Indeed, it follows from the Phillip Hall Theorem (Proposition 1.2.6) and the Euler-Poincaré formula (Theorem 1.2.8) that if the homology of $P$ vanishes below the top dimension then

$$
\tilde{\beta}_{l(P)}(P)=(-1)^{l(P)} \mu(\hat{P}) .
$$

By applying the recursive definition of Möbius function we get the recursive formula observed by Sundaram in [175],

$$
\tilde{\beta}_{l(P)-1}(P \backslash\{\hat{0}\})=\sum_{x \in P}(-1)^{l(P)+r(x)} \tilde{\beta}_{r(x)-2}(\hat{0}, x),
$$

where $P$ is a Cohen-Macaulay poset with a bottom element $\hat{0}$ and $r(x)$ is the rank of $x$.

Since general sequentially Cohen-Macaulay posets can have homology in multiple dimensions, (4.1.1) and (4.1.2) do not hold in the nonpure setting. However, if the sequentially Cohen-Macaulay poset has a property known as semipure, then the following generalization of (4.1.2) shows that Möbius function can still be used to compute its Betti numbers. A poset $P$ is said to be semipure if $P_{\leq y}:=\{x \in P: x \leq y\}$ is pure for all $y \in P$. The proper part of the poset given in Figure 3.2.1 is semipure. Also the face poset of any simplicial complex is semipure.

Theorem 4.1.12 (Wachs [199]). Let $P$ be a semipure sequentially Cohen-Macaulay poset with a bottom element $\hat{0}$. Then for all $m$,

$$
\tilde{\beta}_{m-1}(P \backslash\{\hat{0}\})=\sum_{\substack{x \in P \\ m(x)=m}}(-1)^{m+r(x)} \tilde{\beta}_{r(x)-2}(\hat{0}, x),
$$

where $m(x)$ is the length of the longest chain of $P$ containing $x$.

Exercise 4.1.13. Prove Theorem 4.1.12.

We will demonstrate the effectiveness of the formulas (4.1.2) and (4.1.3) in computing Betti numbers on concrete examples in Section 4.3. Equivariant versions of these formulas will be discussed in Section 4.4. For more general versions see [199].

There is a homotopy version of Cohen-Macaulay complexes due to Quillen [135] and a homotopy version of sequentially Cohen-Macaulay complexes studied by Björner, Wachs and Welker $[\mathbf{4 3}, 44]$. The requirement that homology vanish below a certain dimension is replaced by the requirement that the homotopy groups vanish below that dimension. The homotopy versions are stronger than the homology versions, but are still still weaker than shellability. They are not topological properties, however. For instance there is a triangulation of the 5-sphere that is not homotopy Cohen-Macaulay, see [135, Section 8].

We summarize the implications for bounded posets in the following diagram. 


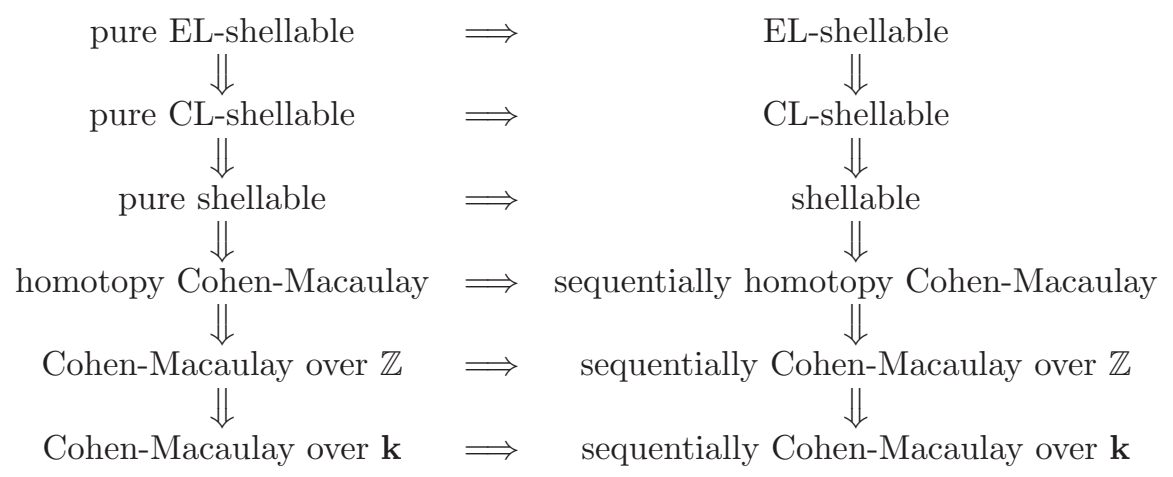

It is known that all the implications but

$$
\text { (pure) EL-shellable } \Longrightarrow \text { (pure) CL-shellable }
$$

are strict. Whether or not there are CL-shellable posets that are not EL-shellable is an open question. It is also unknown whether or not CL-shellability and dual CLshellability are equivalent. Examples of shellable posets that are not CL-shellable were obtained by Vince and Wachs [194] and Walker [206].

There are other important properties of simplicial complexes which have recursive formulations such as vertex decomposability (introduced by Provan and Billera [133] for pure simplicial complexes and extended to nonpure simplicial complexes by Björner and Wachs [41]) and constructible complexes (introduced by Hochster [96] and extended to the nonpure case by Jonsson [103]). There are no special poset versions of these tools, however, as there are for shellability.

\subsection{Recursive atom orderings}

In this section we present a rather technical, but very useful tool for establishing poset shellability (and in turn sequential Cohen-Macaulayness). This technique, called recursive atom ordering, was introduced by Björner and Wachs in the early 1980's. It is equivalent to CL-shellability, but does not involve edge labelings.

Definition 4.2.1. A bounded poset $P$ is said to admit a recursive atom ordering if its length $l(P)$ is 1 , or if $l(P)>1$ and there is an ordering $a_{1}, a_{2}, \ldots, a_{t}$ of the atoms of $P$ that satisfies:

(i) For all $j=1,2, \ldots, t$ the interval $\left[a_{j}, \hat{1}\right]$ admits a recursive atom ordering in which the atoms of $\left[a_{j}, \hat{1}\right]$ that belong to $\left[a_{i}, \hat{1}\right]$ for some $i<j$ come first.

(ii) For all $i<j$, if $a_{i}, a_{j}<y$ then there is a $k<j$ and an atom $z$ of $\left[a_{j}, \hat{1}\right]$ such that $a_{k}<z \leq y$.

A recursive coatom ordering is a recursive atom ordering of the dual poset $P^{*}$.

Figure 4.2.1 (a) gives an example of a poset that does not admit a recursive atom ordering since condition (ii) fails for every ordering of the atoms. The poset in Figure 4.2.1 (b) does admit a recursive atom ordering. The left to right order in the drawing of the Hasse diagram gives an ordering of the atoms that satisfies (i) and (ii), for each interval. 


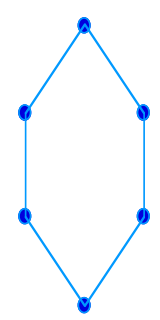

(a)

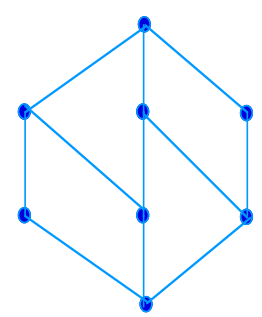

(b)

Figure 4.2.1

Theorem 4.2.2 (Björner and Wachs $[\mathbf{3 8}, 40]$ ). A bounded poset $P$ is $C L$-shellable if and only if $P$ admits a recursive atom ordering.

Proof idea. We use a recursive procedure to obtain a recursive atom ordering from a CL-labeling and vice versa. Given a CL-labeling $\lambda$, order the atoms of $P$ in increasing order of the labels $\lambda(c, \hat{0} \lessdot a)$, where $c$ is any maximal chain containing the atom $a$ (the label is independent of the maximal chain $c$ since $\hat{0}<a$ is a bottom edge). Then recursively use the restriction of the CL-labeling to each interval $[a, \hat{1}]$ to obtain a recursive atom ordering of $[a, \hat{1}]$.

Conversely, given a recursive atom ordering of $P$, label the bottom edge of each maximum chain with the position of the atom in the atom ordering. Then recursively use the recursive atom ordering of $[a, \hat{1}]$ to obtain an appropriate chainedge labeling of $[a, \hat{1}]$, for each atom $a$.

A bounded poset $P$ is said to be semimodular if for all $u, v \in P$ that cover some $x \in P$, there is an element $y \in P$ that covers both $u$ and $v$. Semimodular posets are not necessarily shellable. A poset is said to be totally semimodular if every closed interval is semimodular. Note that semimodular lattices are totally semimodular. In particular, the boolean algebra and the partition lattice are totally semimodular.

Theorem 4.2.3 (Björner and Wachs [38]). Every ordering of the atoms of a totally semimodular poset is a recursive atom ordering.

Theorem 4.2.4 (Björner and Wachs [38]). An ordering of the facets of a simplicial complex $\Delta$ is a shelling if and only if the ordering is a recursive coatom ordering of the face lattice $L(\Delta)$.

Exercise 4.2.5. Prove

(a) Theorem 4.2.3.

(b) Theorem 4.2.4

Definition 4.2.6 (Wachs and Walker $[203]$ ). A meet semilattice $P$ with rank function $r$ is said to be a geometric semilattice if

(i) every interval is a geometric lattice

(ii) for all $x \in P$ and subset $A$ of atoms whose join exists, if $r(x)<r(\bigvee A)$ then there is an $a \in A$ such that $a \not \leq x$ and $a \vee x$ exists.

Examples of geometric semilattices include

- geometric lattices 
- intersection semilattices of affine hyperplane arrangements

- face posets of matroid complexes

- top truncated geometric lattices; i.e., $L_{S}$ where $L$ is a geometric lattice and

$$
S=\{0,1,2, \ldots, j\}
$$

for some $j \leq l(L)$

- $L-[x, \hat{1}]$, where $L$ is a geometric lattice and $x \in L$ (this is a characterization).

A set $A$ of atoms in a geometric semilattice $P$ is said to be independent if $\bigvee A$ exists and $r(\bigvee A)=|A|$. A basic set of atoms is a maximal independent set of atoms.

Theorem 4.2.7 (Wachs and Walker [203]). Any ordering of the atoms of a geometric semilattice $P$ that begins with a basic set of atoms is a recursive atom ordering of $P \cup\{\hat{1}\}$.

Exercise 4.2.8. Let $P$ be a geometric semilattice.

(a) Show $P$ is pure.

(b) Show every principal upper order ideal $P_{\geq x}:=\{y \in P: y \geq x\}$ is a geometric semilattice.

(c) Prove Theorem 4.2.7.

From the proof of Theorem 4.2.2, we know that to every recursive atom ordering of a poset one can associate a CL-labeling. A natural question to ask, when given a particular recursive atom ordering, is whether there is a nice associated CLlabeling. Then one can obtain Betti numbers and bases from the decreasing chains. Ziegler [215] describes such a CL-labeling for one of the recursive atom orderings of Theorem 4.2.7 and shows that it is in fact an EL-labeling. He then constructs bases for homology and cohomology of geometric semilattices which generalize the Björner NBC bases for geometric lattices discussed in Section 3.2.3.

\subsection{More examples}

In this section we present examples of semipure posets that admit recursive atom orderings and we demonstrate the use of the recursive formulas (4.1.2) and (4.1.3) in computing their Betti numbers. Let's begin with some pure examples.

Example 4.3.1. Let $k \leq n$. The injective word poset $\mathcal{I}_{n, k}$ is defined to be the poset of words of length at most $k$ over alphabet $[n]$ with no repeated letters. The order relation is the subword relation. This poset is pure and is a lower order ideal of the normal word poset $\mathcal{N}_{n}$ (discussed in Exercise 3.3.8) which in turn is a subposet of the word poset $\mathcal{W}_{n}$ (also discussed in Exercise 3.3.8). It is left as an exercise to show that lexicographic order on the injective words of length $k$ is a recursive coatom ordering of $\mathcal{I}_{n, k} \cup\{\hat{1}\}$. One can use the fact that all closed intervals of $\mathcal{I}_{n, k}$ are isomorphic to Boolean algebras. Since the duals of the closed intervals are totally semimodular, one needs only to verify condition (i) of Definition 4.2.1.

\section{Exercise 4.3.2.}

(a) Show that lexicographic order on the injective words of length $k$ is a recursive coatom ordering of $\mathcal{I}_{n, k} \cup\{\hat{1}\}$. 
(b) Use formula (4.1.2) to show that the top Betti number $\tilde{\beta}_{k-1}\left(\bar{I}_{n, n}\right)$ is equal to $d_{n}$, the number of derangements of $n$ elements. Consequently, $\bar{I}_{n, n}$ has the homotopy type of a wedge of $d_{n}$ spheres of dimension $k-1$.

(c) Let $\mathcal{N}_{n, k}$ be the induced subposet of $\mathcal{N}_{n}$ consisting of words of length at most $k$. Use Exercise 3.3.8, Theorem 3.4.1, and formula (4.1.2) to show that $\overline{\mathcal{N}}_{n, k}$ has the homotopy type of a wedge of $(n-1)^{k}$ spheres of dimension $k-1$.

(d) Let $\mathcal{W}_{n, k}$ be the induced subposet of $\mathcal{W}_{n}$ consisting of words of length at most $k$. Show that $\overline{\mathcal{W}}_{n, k}$ also has the homotopy type of a wedge of $(n-1)^{k}$ spheres of dimension $k-1$.

Remark 4.3.3. The injective word poset and normal word poset of Example 4.3.1 were introduced by Farmer $[\mathbf{6 8}]$ who showed that $\overline{\mathcal{I}}_{n, k}$ and $\overline{\mathcal{N}}_{n, k}$ have the homology of a wedge of $(k-1)$-spheres. Björner and Wachs [38] recovered these results and strengthened them to homotopy by establishing shellability. The Betti number computations of Exercise 4.3.2 (b) and (c) are due to Reiner and Webb [140] and Farmer $[\mathbf{6 8}]$, respectively.

Recall that in Example 3.2.2, we used EL-shellability to show that the proper part of the partition lattice $\Pi_{n}$ has the homotopy type of a wedge of $(n-1)$ ! spheres of dimension $n-3$. In the next two examples we use the techniques of Sections 4.1 and 4.2 to obtain analogous results for the even and odd block size partition posets.

Example 4.3.4. Let $\Pi_{2 n}^{\text {even }}$ be the subposet of $\Pi_{2 n}$ consisting of partitions whose block sizes are even. The even block size partition poset is pure but lacks a bottom element. The upper intervals $[x, \hat{1}]$ are all partition lattices, which are totally semimodular; so every atom ordering of $[x, \hat{1}]$ is a recursive atom ordering. Associate a word with each atom of $\Pi_{2 n}^{\text {even }} \cup\{\hat{0}\}$ by listing the elements of each block in increasing order and then listing the blocks in lexicographic order. We claim that lexicographic order on the words corresponds to a recursive atom ordering of $\Pi_{2 n}^{\text {even }} \cup\{\hat{0}\}$. The verification is left as an exercise.

We next use (4.1.2) to compute the Betti numbers of $\bar{\Pi}_{2 n}^{\text {even }}$. Since $\Pi_{2 n}^{\text {even }}$ is pure and the upper intervals are isomorphic to partition lattices, to compute the unique nonvanishing Betti number $\tilde{\beta}_{n-2}\left(\bar{\Pi}_{2 n}^{\text {even }}\right)$, we apply (4.1.2) to the dual of the poset. Let $\beta_{2 n}$ denote the Betti number $\tilde{\beta}_{n-2}\left(\bar{\Pi}_{2 n}^{\text {even }}\right)$. By $(4.1 .2)$ we have

$$
\begin{aligned}
\beta_{2 n} & =\sum_{x}(-1)^{b(x)+n} \tilde{\beta}_{b(x)-3}(x, \hat{1}) \\
& =\sum_{x}(-1)^{b(x)+n}(b(x)-1) ! \\
& =\sum_{r=1}^{n}(-1)^{r+n}(r-1) !\left|\left\{x \in \Pi_{2 n}^{\text {even }}: b(x)=r\right\}\right|,
\end{aligned}
$$

where $b(x)$ denotes the number of blocks of $x$. Note that

$$
\left|\left\{x \in \Pi_{2 n}^{\text {even }}: b(x)=r\right\}\right|=\frac{1}{r !} \sum_{\left(j_{1}, j_{2}, \ldots, j_{r}\right) \models n}\left(\begin{array}{c}
2 n \\
2 j_{1}, 2 j_{2}, \ldots, 2 j_{r}
\end{array}\right),
$$

where $\vDash n$ denotes composition of $n$. We now have

$$
\beta_{2 n}=\sum_{r=1}^{n}(-1)^{r+n} \frac{1}{r} \sum_{\left(j_{1}, j_{2}, \ldots, j_{r}\right) \models n}\left(\begin{array}{c}
2 n \\
2 j_{1}, 2 j_{2}, \ldots, 2 j_{r}
\end{array}\right) .
$$


The exponential generating function for the Betti numbers is thus given by,

$$
\begin{aligned}
\sum_{n \geq 1} \beta_{2 n} \frac{u^{2 n}}{(2 n) !} & =\sum_{r \geq 1}(-1)^{r} \frac{1}{r} \sum_{n \geq 1} \sum_{\left(j_{1}, j_{2}, \ldots, j_{r}\right) \models n}\left(\begin{array}{c}
2 n \\
2 j_{1}, 2 j_{2}, \ldots, 2 j_{r}
\end{array}\right)(-1)^{n} \frac{u^{2 n}}{(2 n) !} \\
& =\sum_{r \geq 1}(-1)^{r} \frac{1}{r}\left(\sum_{j \geq 1}(-1)^{j} \frac{u^{2 j}}{(2 j) !}\right)^{r} \\
& =-\ln (\cos u) .
\end{aligned}
$$

By taking derivatives we get,

$$
\sum_{n \geq 1} \beta_{2 n} \frac{u^{2 n-1}}{(2 n-1) !}=\tan u .
$$

So $\beta_{2 n}$ is equal to the coefficient of $\frac{u^{2 n-1}}{(2 n-1) !}$ in the Taylor series expansion of $\tan u$. It is well known that this coefficient is equal to the Euler number $E_{2 n-1}$, where $E_{m}$ is defined to be the number of alternating permutations in $\mathfrak{S}_{m}$, i.e., permutations with descents at all the even positions and ascents at all the odd positions. We conclude that $\bar{\Pi}_{2 n}^{\text {even }}$ has the homotopy type of a wedge of $E_{2 n-1}$ spheres of dimension $n-2$.

There is an alternative way to arrive at $\beta_{2 n}=E_{2 n-1}$. Replace the last line of (4.3.1) with

$$
-\ln \left(\sum_{j \geq 0}(-1)^{j} \frac{u^{2 j}}{(2 j) !}\right)
$$

and then take derivatives of both sides of the equation. This results in

$$
\sum_{n \geq 1} \beta_{2 n} \frac{u^{2 n-1}}{(2 n-1) !} \sum_{j \geq 0}(-1)^{j} \frac{u^{2 j}}{(2 j) !}=\sum_{j \geq 0}(-1)^{j-1} \frac{u^{2 j-1}}{(2 j-1) !} .
$$

By equating coefficients we obtain the recurrence relation,

$$
\sum_{r=1}^{j}\left(\begin{array}{l}
2 j-1 \\
2 r-1
\end{array}\right)(-1)^{r} \beta_{2 r}=-1
$$

It is not difficult to check that $E_{2 r-1}$ satisfies the same recurrence relation.

Example 4.3.5. Let $\Pi_{2 n+1}^{\text {odd }}$ be the subposet of $\Pi_{2 n+1}$ consisting of partitions whose block sizes are odd. The odd block size partition poset is totally semimodular; so any atom ordering is a recursive atom ordering.

Now let $\beta_{2 n+1}$ denote the top Betti number $\tilde{\beta}_{n-2}\left(\bar{\Pi}_{2 n+1}^{\text {odd }}\right)$. An argument similar to the one used in deriving (4.3.1) yields: $\sum_{n \geq 0} \beta_{2 n+1} \frac{u^{2 n+1}}{(2 n+1) !}$ is the compositional inverse of $\sum_{n \geq 0}(-1)^{n} \frac{u^{2 n+1}}{(2 n+1) !}$, i.e.,

$$
\sum_{n \geq 0} \beta_{2 n+1} \frac{1}{(2 n+1) !}\left(\sum_{j \geq 0}(-1)^{j} \frac{u^{2 j+1}}{(2 j+1) !}\right)^{2 n+1}=u .
$$

Since $\sum_{j \geq 0}(-1)^{j} \frac{u^{2 j+1}}{(2 j+1) !}=\sin u$, we have $\sum_{n \geq 0} \beta_{2 n+1} \frac{u^{2 n+1}}{(2 n+1) !}=\sin ^{-1} u$. The coefficient of $\frac{u^{2 n+1}}{(2 n+1) !}$ in the Taylor series expansion of $\sin ^{-1} u$ is $(2 n+1) ! !^{2}$, where $(2 n+1) ! !:=1 \cdot 3 \cdot 5 \cdots(2 n+1)$. We conclude that $\bar{\Pi}_{2 n+1}^{\text {odd }}$ has the homotopy type of a wedge of $(2 n+1) ! !^{2}$ spheres of dimension $n-2$. 
Exercise 4.3.6. For integers $n, d \geq 1$ and $k \geq 0$, let $\Pi_{n d+k}^{k \bmod d}$ be the subposet of the partition lattice $\Pi_{n d+k}$ consisting of partitions whose block sizes are congruent to $k \bmod d$. This poset is pure only for $k \equiv 0$ or $1 \bmod d$, and is bounded only for $k \equiv 1 \bmod d$.

(a) Show that lexicographical order on the words associated with the atoms as in Example 4.3 .4 (i.e., list the elements of each block in increasing order and then list the blocks lexicographical order) yields a recursive atom ordering of $\Pi_{n d}^{0 \bmod d} \cup\{\hat{0}\}$.

(b) Show that $\Pi_{n d+1}^{1 \text { mod } d}$ admits a recursive atom ordering.

(c) Find a recursive atom ordering of $\Pi_{n d+k}^{k \bmod d} \cup\{\hat{0}\}$ for $k \not \equiv 1 \bmod d$.

(d) Show that $\bar{\Pi}_{n d}^{0 \text { mod } d}$ has the homotopy type of a wedge of $E_{d n-1}^{d}$ spheres of dimension $n-2$, where

$$
E_{d n-1}^{d}=\mid\left\{\sigma \in \mathfrak{S}_{n d-1}: \operatorname{des}(\sigma)=\{d, 2 d, \ldots,(n-1) d\} \mid .\right.
$$

(e) Show that $\bar{\Pi}_{n d+1}^{1}$ mod $d$ has the homotopy type of a wedge of $c_{n}$ spheres of dimension $n-2$, where $\sum_{n \geq 0} c_{n} \frac{u^{n d+1}}{(n d+1) !}$ is the compositional inverse of $\sum_{n \geq 0}(-1)^{n} \frac{u^{n d+1}}{(n d+1) !}$.

Remark 4.3.7. The result on the Möbius invariant of the even block size partition lattice derived in Example 4.3.4 first appeared in in the 1976 MIT thesis of Garrett Sylvestor on Ising ferromagnets [186]. Stanley [165] extended this result to the $d$ divisible partition lattice of Exercise 4.3.6 (d). The Möbius invariant result derived in Example 4.3.5 and Exercise 4.3.6 (e) are also due to Stanley. The recursive atom ordering for the $d$-divisible partition lattice (Example 4.3.4 and Exercise 4.3.6 (a)) is due to Wachs (see [148]), as is an EL-labeling of this poset [196]. The recursive atom ordering for the $1 \mathrm{mod} d$ partition lattice (Example 4.3.5 and Exercise 4.3.6 (b)) is due to Björner (see [54] and [38]). The general recursive atom ordering of $\Pi_{n d+k}^{k \bmod d} \cup\{\hat{0}\}$ of Exercise 4.3.6 (c) is due to Wachs [199].

In the next example, we demonstrate the full power of Theorem 4.1 .12 by finding the Betti numbers for a nonpure shellable poset.

Example 4.3.8. For $n \geq k \geq 3$, let $\Pi_{n}^{\geq k}$ be the subposet of $\Pi_{n}$ consisting of partitions whose block sizes are at least $k$. It was shown by Björner and Wachs [40] that the poset $\Pi_{\bar{n}}^{\geq k} \cup\{\hat{0}\}$ admits a recursive atom ordering similar to that of $\Pi_{2 n}^{\text {even }} \cup\{\hat{0}\}$. The following computation of Betti numbers appears in [199]. The dual of $\Pi_{\bar{n}}^{\geq k}$ is semipure and the upper intervals are partition lattices; so we apply (4.1.3) to the dual. First note that

$$
m(x)=\sum_{i=1}^{b(x)}\left\lfloor\frac{\left|B_{i}\right|}{k}\right\rfloor-1,
$$

where $B_{1}, B_{2}, \ldots, B_{b(x)}$ are the blocks of $x$. By (4.1.3) we have,

$$
\begin{aligned}
\tilde{\beta}_{m-2}\left(\bar{\Pi}_{n}^{\geq k}\right) & =\sum_{x: m(x)=m-1}(-1)^{b(x)+m} \tilde{\beta}_{b(x)-3}(x, \hat{1}) \\
& =\sum_{r \geq 1}(-1)^{r+m}(r-1) !\left|\left\{x \in \Pi_{\bar{n}}^{\geq k}: b(x)=r, m(x)=m-1\right\}\right| .
\end{aligned}
$$


We have,

$$
\left|\left\{x \in \Pi_{n}^{\geq n}: b(x)=r, m(x)=m-1\right\}\right|=\frac{1}{r !} \sum_{\substack{\left(j_{1}, j_{2}, \ldots, j_{r}\right) \vDash n \\
j_{j} \geq k \forall i \\
\sum_{i=1}^{r}\left\lfloor j_{i} / k\right\rfloor=m}}\left(\begin{array}{c}
n \\
j_{1}, j_{2}, \ldots, j_{r}
\end{array}\right) .
$$

The two parameter exponential generating function is thus,

$$
\begin{aligned}
& \sum_{m, n \geq 1}(-1)^{m} \tilde{\beta}_{m-2}\left(\bar{\Pi}_{n}^{\geq k}\right) t^{m} \frac{u^{n}}{n !} \\
& =\sum_{r \geq 1}(-1)^{r} \frac{1}{r} \sum_{n, m \geq 1} \sum_{\substack{\left(j_{1}, j_{2}, \ldots, j_{r}\right) \vDash n \\
j_{i} \geq k \forall i}}\left(\begin{array}{c}
n \\
j_{1}, j_{2}, \ldots, j_{r}
\end{array}\right) t^{\sum_{i=1}^{r}\left\lfloor j_{i} / k\right\rfloor} \frac{u^{n}}{n !} \\
& =\sum_{r \geq 1}(-1)^{r} \frac{1}{r}\left(\sum_{j \geq k} t^{\lfloor j / k\rfloor} \frac{u^{j}}{j !}\right)^{r} \\
& =-\ln \left(1+\sum_{j \geq k} t^{\lfloor j / k\rfloor} \frac{u^{j}}{j !}\right) .
\end{aligned}
$$

Exercise 4.3.9. Show that $\Pi_{\bar{n}}^{\geq k} \cup\{\hat{0}\}$ has a recursive atom ordering.

Problem 4.3.10. Linusson [119] computed the Möbius invariant of the poset $\Pi_{\bar{n}}^{\geq k} \cup\{\hat{0}\}$ in order to compute lower bounds for the complexity of a problem similar to the $k$-equal problem of Section 3.2.4; namely that of determining whether a given list of real numbers has the property that the number of occurrences of each entry is at least $k$. It was shown by Björner and Lovász [33] that Betti number computations give better bounds than Möbius function computations. Can the Betti number computation of Example 4.3 .8 be used to improve Linusson's lower bound for the complexity of the "at least k problem"?

Exercise 4.3.11 (Wachs [199]). In this exercise, we generalize the results of Examples 4.3.4 and 4.3.5 to the nonpure case of Exercise 4.3.6 . For positive integers $n, d, k$, with $k \leq d$, let $k_{0}=k / \operatorname{gcd}(k, d)$ and $d_{0}=d / \operatorname{gcd}(k, d)$. Show that

$$
\sum_{\substack{m \geq 1 \\ n \geq 0}}(-1)^{m} \beta_{m-2}\left(\bar{\Pi}_{n d+k}^{k \bmod d}\right) t^{m d_{0}+1} \frac{u^{n d+k}}{(n d+k) !}=f\left(\sum_{i \geq 0} t^{\left\lfloor i / k_{0}\right\rfloor d_{0}+1} \frac{u^{i d+k}}{(i d+k) !}\right),
$$

where $f(y)$ is the compositional inverse of the formal power series

$$
g(y)=\sum_{i \geq 0} \frac{y^{i d_{0}+1}}{\left(i d_{0}+1\right) !}
$$

\subsection{The Whitney homology technique}

In this section we discuss a technique for computing group representations on the homology of sequentially Cohen-Macaulay posets and demonstrate its use on the examples of Section 4.3. This technique was introduced by Sundaram [175] in the pure case and later generalized to semipure posets by Wachs [199]. The pure case is based on an equivariant version of (4.1.2) and the semipure version is based on the more general Theorem 4.1.12. 
For any Cohen-Macaulay $G$-poset $P$ with bottom element $\hat{0}$, Whitney homology of $P$ is defined for each integer $r$ as follows,

$$
\mathrm{WH}_{r}(P)=\bigoplus_{x \in P_{r}} \tilde{H}_{r-2}(\hat{0}, x)
$$

where $P_{r}:=\{x \in P: r(x)=r\}$.

The action of $G$ on $P$ induces a representation of $G$ on $\mathrm{WH}_{r}(P)$. Indeed $g \in G$ takes $(r-2)$-chains of $(\hat{0}, x)$ to $(r-2)$-chains of $(\hat{0}, g x)$. More precisely, as a $G$ module

$$
\mathrm{WH}_{r}(P)=\bigoplus_{x \in P_{r} / G} \tilde{H}_{r-2}(\hat{0}, x) \uparrow_{G_{x}}^{G},
$$

where $P_{r} / G$ is a set of orbit representatives and $G_{x}$ is the stabilizer of $x$.

Whitney homology for geometric lattices was introduced by Baclawski [11] as the homology of an algebraic complex whose Betti numbers are the signless Whitney numbers of the first kind. The formulation given here is due to Björner [22]. Whitney homology for intersection lattices of complex hyperplane arrangements forms an algebra isomorphic to an algebra that Orlik and Solomon used to give a combinatorial presentation of the cohomology algebra of the complement of the arrangement; see [28]. Sundaram [175] recognized that Whitney homology for any Cohen-Macaulay poset could be used as a tool in computing group representations on homology. Her technique is based on the following result, which she obtains as a consequence of the Hopf trace formula.

Theorem 4.4.1 (Sundaram $[\mathbf{1 7 5}, \mathbf{1 7 6}])$ ). Suppose $P$ is a $G$-poset with a bottom element $\hat{0}$. Then

$$
\bigoplus_{r=-1}^{l(P)-1}(-1)^{r} \tilde{H}_{r}(P \backslash\{\hat{0}\}) \cong_{G} \bigoplus_{r=0}^{l(P)}(-1)^{r-1} \bigoplus_{x \in P / G} \tilde{H}_{r-2}(\hat{0}, x) \uparrow_{G_{x}}^{G} .
$$

Consequently, if $P$ is also Cohen-Macaulay,

$$
\tilde{H}_{l(P)-1}(P \backslash\{\hat{0}\}) \cong_{G} \bigoplus_{r=0}^{l(P)}(-1)^{l(P)+r} \mathrm{WH}_{r}(P) .
$$

\section{Exercise 4.4.2.}

(a) Prove Theorem 4.4.1.

(b) Show that if $P$ is the face poset of a simplicial complex then (4.4.1) reduces to the Hopf trace formula.

Example 4.4.3 (Reiner and Webb [140]). Consider the injective word poset $\mathcal{I}_{n, k}$ of Example 4.3.1. Let $G$ be the symmetric group $\mathfrak{S}_{n}$, which acts on $\mathcal{I}_{n, k}$ in the obvious way. Since $\mathcal{I}_{n, k}$ is Cohen-Macaulay, we can apply (4.4.2). Let $x \in \mathcal{I}_{n, k}$ be a word of length $r$. Clearly $G_{x}$ is isomorphic to the Young subgroup $\left(\mathfrak{S}_{1}\right)^{\times r} \times \mathfrak{S}_{n-r}$, since the letters of $x$ must be fixed and the letters outside of $x$ may be freely permuted. $G_{x}$ acts trivially on the letters outside of $x$. The interval $(\hat{0}, x)$ is isomorphic to the proper part of the Boolean algebra $B_{r}$; so its top homology is 1-dimensional. Hence,

$$
\tilde{H}_{r-2}(\hat{0}, x) \cong_{G_{x}} \underbrace{S^{(1)} \otimes \cdots \otimes S^{(1)}}_{r} \otimes S^{(n-r)}
$$


Since $\mathfrak{S}_{n}$ acts transitively on each rank row of $\mathcal{I}_{n, k}$, we have

$$
\begin{aligned}
\mathrm{WH}_{r}\left(\mathcal{I}_{n, k} \cup\{\hat{1}\}\right) & =\tilde{H}_{r-2}(\hat{0}, x) \uparrow \uparrow_{G_{x}}^{\mathfrak{S}_{n}} \\
& \cong \mathfrak{S}_{n} \quad\left(S^{(1)}\right)^{\bullet r} \bullet S^{(n-r)} .
\end{aligned}
$$

It follows from (4.4.2) that

$$
\begin{aligned}
\tilde{H}_{k-1}\left(\overline{\mathcal{I}}_{n, k}\right) & \cong_{\mathfrak{S}_{n}} \bigoplus_{r=0}^{k}(-1)^{k-r}\left(S^{(1)}\right)^{\bullet r} \bullet S^{(n-r)} \\
& \cong_{\mathfrak{S}_{n}}(-1)^{k} S^{(n)} \oplus S^{(1)} \bullet \bigoplus_{r=0}^{k-1}(-1)^{k-1-r}\left(S^{(1)}\right)^{\bullet r} \bullet S^{(n-r-1)} \\
& \cong_{\mathfrak{S}_{n}}(-1)^{k} S^{(n)} \oplus S^{(1)} \bullet \tilde{H}_{k-2}\left(\overline{\mathcal{I}}_{n-1, k-1}\right) .
\end{aligned}
$$

This recurrence relation, for $n=k$, is an equivariant version of the well-known recurrence relation for derangement numbers: $d_{n}=(-1)^{n}+n d_{n-1}$. From this recurrence relation, one can obtain the following decomposition of $\tilde{H}_{n-1}\left(\overline{\mathcal{I}}_{n, n}\right)$ into irreducibles:

$$
\tilde{H}_{n-1}\left(\overline{\mathcal{I}}_{n, n}\right) \cong \mathfrak{S}_{n} \bigoplus_{\lambda \vdash n} c_{\lambda} S^{\lambda},
$$

where $c_{\lambda}$ is the number of standard Young tableaux of shape $\lambda$ whose first descent is even.

Exercise 4.4.4. Use Exercise 2.2.10 and the recurrence relation to prove (4.4.3).

Note that by taking dimensions on both sides of (4.4.3), and applying the wellknown Robinson-Schensted-Knuth correspondence, one recovers an enumerative result of Désarménien [59] that the number of derangements in $\mathfrak{S}_{n}$ is equal to the number of permutations in $\mathfrak{S}_{n}$ with first descent even. Désarménien gives an elegant direct combinatorial proof of this result. The Frobenius characteristic of the representation $\bigoplus_{\lambda \vdash n} c_{\lambda} S^{\lambda}$ was used by Désarménien and Wachs [60] to obtain deeper enumerative connections between the two classes of permutations. A refinement of the Reiner-Webb decomposition was given by Hanlon and Hersh [89]. In [139], Reiner and Wachs use (4.4.3) to obtain a decomposition (into irreducible representations of $\mathfrak{S}_{n}$ ) of the eigenspaces of the so called "random to top" operator in card shuffling theory. Type B analogs of these enumerative and representation theoretic results can also be found in [139].

Exercise 4.4.5. Recall from Exercises 4.3.2 (c) and (d) that the top Betti number of both $\overline{\mathcal{N}}_{n+1, k}$ and $\overline{\mathcal{W}}_{n+1, k}$ is $n^{k}$.

(a) (Shareshian and Wachs) Use (4.4.2) to prove

$$
\operatorname{ch}\left(\tilde{H}_{n}\left(\overline{\mathcal{N}}_{n+1, k}\right) \downarrow_{\mathfrak{S}_{n}}^{\mathfrak{S}_{n+1}}\right)=\sum_{t \geq 1} S(k, t) h_{1}^{t} h_{n-t},
$$

where $S(n, k)$ is the Stirling number of the second kind.

(b) Conclude that $\tilde{H}_{n}\left(\overline{\mathcal{N}}_{n+1, k}\right) \downarrow_{\mathfrak{S}_{n}}^{\mathfrak{S}_{n+1}}$ is the $k$ th tensor power of $S^{(n)} \oplus S^{(n-1,1)}$ by comparing characters.

(c) Show (a) and (b) hold for $\mathcal{W}_{n+1, k}$. (Part (b) for $\mathcal{N}_{n+1, k}$ and $\mathcal{W}_{n+1, k}$ was originally observed by Stanley by means of the fixed point Möbius invariant, see Section 4.6.) 
Exercise 4.4.6 (Sundaram [175]). Show that by applying (4.4.2) to the Boolean algebra $B_{n}$, one obtains the well-known symmetric function identity,

$$
\sum_{i=0}^{n}(-1)^{i} e_{i} h_{n-i}=0 .
$$

Sundaram developed her Whitney homology technique in order to study representations of the symmetric group on various Cohen-Macaulay subposets of the partition lattice. In fact, she applies it to the full partition lattice and obtains a conceptual representation theoretic proof of the following classical result of Stanley. The original proof of Stanley used a computation, due to Hanlon [85], of the fixed point Möbius invariant of the partition lattice. This technique is discussed in Section 4.6 .

Theorem 4.4.7 (Stanley [167]). For all positive integers $n$,

$$
\left.\tilde{H}_{n-3}\left(\bar{\Pi}_{n}\right)\right) \cong \mathfrak{S}_{n} e^{2 \pi i / n} \uparrow_{\mathfrak{C}_{n}}^{\mathfrak{S}_{n}} \otimes \operatorname{sgn}_{n}
$$

where $\mathfrak{C}_{n}$ is the cyclic subgroup of $\mathfrak{S}_{n}$ generated by $\sigma:=(1,2, \ldots, n)$ and $e^{2 \pi i / n}$ denotes the one dimensional representation of $\mathfrak{C}_{n}$ whose character value at $\sigma$ is $e^{2 \pi i / n}$

Proof. (Sundaram [175]). Theorem 4.4.1 is applied to the dual of the partition lattice. By setting $T=\mathbb{Z}^{+}, b=r+1$ and $z_{i}=1$ in (2.4.3), one obtains

$$
\sum_{n>r} \operatorname{chWH}_{r}\left(\left(\Pi_{n}\right)^{*}\right)=\operatorname{ch} \tilde{H}_{r-2}\left(\bar{\Pi}_{r+1}\right)\left[\sum_{i \geq 1} h_{i}\right],
$$

Now (4.4.2) yields,

$$
\begin{aligned}
\sum_{n \geq 1}(-1)^{n-1} \operatorname{ch} \tilde{H}_{n-2}\left(\Pi_{n} \backslash\{\hat{1}\}\right) & =\sum_{n \geq 1} \sum_{r=0}^{n-1}(-1)^{r} \operatorname{chWH}_{r}\left(\left(\Pi_{n}\right)^{*}\right) \\
& =\sum_{r \geq 0}(-1)^{r} \operatorname{ch} \tilde{H}_{r-2}\left(\bar{\Pi}_{r+1}\right)\left[\sum_{i \geq 1} h_{i}\right] .
\end{aligned}
$$

Since $\Delta\left(\Pi_{n} \backslash\{\hat{1}\}\right)$ is contractible for all $n>1$ and is $\{\emptyset\}$ when $n=1$, it follows that

$$
h_{1}=\sum_{r \geq 0}(-1)^{r} \operatorname{ch} \tilde{H}_{r-2}\left(\bar{\Pi}_{r+1}\right)\left[\sum_{i \geq 1} h_{i}\right] .
$$

Since $h_{1}$ is the plethystic identity,

$$
\begin{aligned}
\sum_{r \geq 1}(-1)^{r-1} \operatorname{ch} \tilde{H}_{r-3}\left(\bar{\Pi}_{r}\right) & =\left(\sum_{i \geq 1} h_{i}\right)^{[-1]} \\
& =\sum_{d \geq 1} \frac{1}{d} \mu(d) \log \left(1+p_{d}\right)
\end{aligned}
$$

where [-1] denotes plethystic inverse, $\mu$ is the number theoretic Möbius function, and $p_{d}$ is the power sum symmetric function. The last equation follows from a formula of Cadogan [53], which is also derived in [175]. By extracting the degree $n$ term, we have

$$
\operatorname{ch} \tilde{H}_{n-3}\left(\bar{\Pi}_{n}\right)=\frac{1}{n} \sum_{d \mid n}(-1)^{n-n / d} \mu(d) p_{d}^{n / d} .
$$


A standard formula for the character of an induced representation yields

$$
\operatorname{ch} e^{2 \pi i / n} \uparrow_{\mathfrak{C}_{n}}^{\mathfrak{S}_{n}}=\frac{1}{n} \sum_{d \mid n} \mu(d) p_{d}^{n / d} .
$$

By (2.4.1) and Theorem 2.4.3 (b), this together with (4.4.7) implies (4.4.4), as desired.

The representation $e^{2 \pi i / n} \uparrow_{\mathfrak{C}_{n}}^{\mathfrak{S}_{n}}$ is a well-studied representation called the Lie representation because it is isomorphic to the representation of the symmetric group on the multilinear component of the free Lie algebra on $n$ generators, cf., Theorem 1.6.2.

Exercise 4.4.8 (Sundaram $[\mathbf{1 7 5}]$ ). Use the Whitney homology technique to prove the following results of Calderbank, Hanlon and Robinson [54].

(a) (equivariant version of Exercise 4.3.6 (e))

$$
\sum_{n \geq 0}(-1)^{n} \operatorname{ch} \tilde{H}_{n-2}\left(\bar{\Pi}_{n d+1}^{1 \bmod d}\right)=\left(\sum_{i \geq 0} h_{i d+1}\right)^{[-1]} .
$$

(b) (equivariant version of Exercise 4.3.6 (d))

$$
\sum_{n \geq 1}(-1)^{n-1} \operatorname{ch} \tilde{H}_{n-2}\left(\bar{\Pi}_{n d}^{0 \bmod d}\right)=\left(\sum_{i \geq 1} h_{i}\right)^{[-1]}\left[\sum_{i \geq 1} h_{i d}\right] .
$$

We now present an equivariant version of Theorem 4.1.12, which extends Theorem 4.4.1 to the nonpure setting. For any semipure sequentially Cohen-Macaulay $G$-poset $P$ with a bottom element 0 , define $r, m$-Whitney homology to be the $G$ module

$$
\mathrm{WH}_{r, m}(P):=\bigoplus_{\substack{x \in P_{r} \\ m(x)=m}} \tilde{H}_{r-2}(\hat{0}, x),
$$

where $m(x)$ is the length of the longest chain of $P$ containing $x$ and $P_{r}:=\{x \in P$ : $r(x)=r\}$.

Theorem 4.4.9 (Wachs [199]). Let $P$ be a semipure sequentially Cohen-Macaulay $G$-poset. Then for all $m$,

$$
\tilde{H}_{m-1}(P \backslash\{\hat{0}\}) \cong_{G} \bigoplus_{r=0}^{m}(-1)^{m+r} \mathrm{WH}_{r, m}(P) .
$$

Example 4.4.10 (equivariant version of Example 4.3.8 [199]). We will use Theorem 4.4.9 and (2.4.3) to obtain the following formula for the two parameter generating function for the homology of $\bar{\Pi}_{\bar{n}}^{\geq k}$,

$$
\sum_{\substack{m \geq 1 \\ n \geq k}}(-1)^{m-1} \operatorname{ch} \tilde{H}_{m-2}\left(\bar{\Pi}_{n}^{\geq k}\right) u^{n} t^{m}=\left(\sum_{i \geq 1} h_{i}\right)^{[-1]}\left[\sum_{i \geq k} h_{i} u^{i} t^{\left\lfloor\frac{i}{k}\right\rfloor}\right] .
$$

We apply (4.4.9) to the dual of $\Pi_{\bar{n}}^{\geq k}$,

$$
\mathrm{WH}_{r, m}\left(\left(\Pi_{n}^{\geq k}\right)^{*}\right)=\bigoplus_{\substack{\lambda \in \operatorname{Par}(T, r+1) \\ \lambda \vdash n \\ m(\lambda)=m}} \bigoplus_{\substack{x \in \Pi(\lambda) \\ m}} \tilde{H}_{r-2}(x, \hat{1}),
$$


where $T=\{k, k+1, \ldots\}, m(\lambda)=\sum_{i}\left\lfloor\lambda_{i} / k\right\rfloor-1$ (recall (4.3.3) here), and the remaining notation is defined in Example 2.4.9 . By setting $z_{i}=u^{i} t^{\lfloor i / k\rfloor}$ in (2.4.3), we obtain

$$
\begin{aligned}
\sum_{m, n} \operatorname{chWH}_{r, m-1}\left(\left(\Pi_{n}^{\geq k}\right)^{*}\right) u^{n} t^{m} & =\sum_{\lambda \in \operatorname{Par}(T, r+1)}\left(\operatorname{ch} \bigoplus_{x \in \Pi(\lambda)} \tilde{H}_{r-2}(x, \hat{1})\right) u^{|\lambda|} t^{\sum_{i}\left\lfloor\lambda_{i} / k\right\rfloor} \\
& =\operatorname{ch} \tilde{H}_{r-2}\left(\Pi_{r+1}\right)\left[\sum_{i \geq k} h_{i} u^{i} t^{\left\lfloor\frac{i}{k}\right\rfloor}\right] .
\end{aligned}
$$

Thus formula (4.4.11) follows from (4.4.10) and (4.4.6).

The following result generalizes (4.4.8) and (4.4.11). Its proof is similar to that of (4.4.11) described above.

Theorem 4.4.11 (Wachs [199]). Suppose $S \subseteq\{2,3, \ldots\}$ is such that $S$ and $\{s-$ $\min S: s \in S\}$ are closed under addition. For $n \in S$, let $\Pi_{n}^{S}$ be the subposet of $\Pi_{n}$ consisting of partitions whose block sizes are in $S$. Then

$$
\sum_{\substack{m \geq 1 \\ n \in S}}(-1)^{m-1} \operatorname{ch} \tilde{H}_{m-2}\left(\bar{\Pi}_{n}^{S}\right) u^{n} t^{m}=\left(\sum_{i \geq 1} h_{i}\right)^{[-1]}\left[\sum_{i \in S} h_{i} u^{i} t^{\phi(i)}\right],
$$

where $\phi(i):=\max \left\{j \in \mathbb{Z}^{+}: i-(j-1) \min S \in S\right\}$.

We remark that the restricted block size partition poset $\Pi_{n}^{S} \cup\{\hat{0}\}$ of Theorem 4.4.11 is the intersection lattice of a subspace arrangement, which is discussed further in Section 5.4 .

Example 4.4.12 (equivariant version of Exercise 4.3.11 [199]). Theorem 4.4.9 and a generalization of (2.4.3) given in [199] can be used to obtain the following formula for the two parameter generating function for the homology of the $j \bmod d$ partition poset for $j=2,3, \ldots, d$ :

$$
\begin{aligned}
\sum_{\substack{m \geq 1 \\
n \geq 0}}(-1)^{m} & \operatorname{ch} \tilde{H}_{m-1}\left(\bar{\Pi}_{n d+j}^{j \bmod d}\right) u^{n d+j} t^{m d_{0}+1} \\
& =\left(\sum_{i \geq 0} h_{i d_{0}+1}\right)^{[-1]}\left[\sum_{i \geq 0} h_{i d+j} u^{i d+j} t^{\left\lfloor\frac{i}{j_{0}}\right\rfloor d_{0}+1}\right],
\end{aligned}
$$

where $j_{0}=\frac{j}{\operatorname{gcd}(j, d)}$ and $d_{0}=\frac{d}{\operatorname{gcd}(j, d)}$.

Problem 4.4.13. Do the results of this section on restricted block size partition lattices have nice generalizations to Dowling lattices or intersection lattices of Coxeter arrangements?

For other restricted block size partition posets with very interesting equivariant homology, see the work of Sundaram [180].

\subsection{Bases for the restricted block size partition posets}

As we have seen in previous lectures, the construction of explicit bases for homology and cohomology is an effective tool in studying group representations on homology. In this section we construct bases for the homology and cohomology of the restricted 


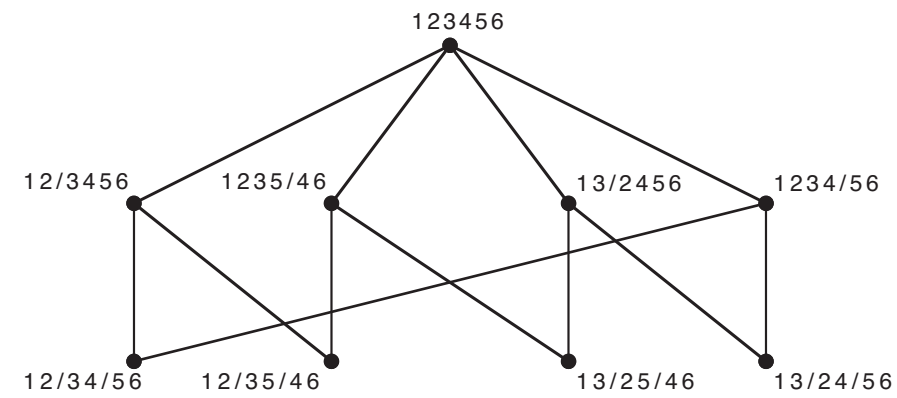

Figure 4.5.1. $\Pi_{123456}^{2}$

block size partition posets studied in the previous section. These bases are used to obtain further results on the representations of the symmetric group on homology of the posets and to relate these representations to representations on homology of certain interesting graph complexes.

\subsubsection{The $d$-divisible partition lattice $\Pi_{n d}^{0 \bmod d}$}

We construct analogs of the splitting basis for the homology of the partition lattice given Section 3.2.2 and its dual basis for cohomology. To switch-split a permutation $\sigma$ at position $j$ is to form the partition

$$
\sigma(1), \ldots, \sigma(j-1), \sigma(j+1) \mid \sigma(j), \sigma(j+2) \ldots, \sigma(n)
$$

of $[n]$. Switch-splitting a permutation at two or more nonadjacent positions is defined similarly. Let $d \geq 2$. For each $\sigma \in \mathfrak{S}_{n d}$, let $\Pi_{\sigma}^{d}$ be the induced subposet of $\Pi_{n d}^{0 \bmod d}$ consisting of partitions obtained by splitting or switch-splitting $\sigma$ at any number of positions in $\{d, 2 d, \ldots, n d\}$. The subposet $\Pi_{123456}^{2}$ of $\Pi_{6}^{0} \bmod 2$ is shown in Figure 4.5.1.

Each poset $\Pi_{\sigma}^{d} \cup\{\hat{0}\}$ is isomorphic to the face lattice $C_{n-1}$ of the $(n-1)$-crosspolytope. Therefore $\Delta\left(\bar{\Pi}_{\sigma}^{d}\right)$ is an $(n-2)$-sphere embedded in $\Delta\left(\bar{\Pi}_{n d}^{0 \bmod d}\right)$, and hence it determines a fundamental cycle $\rho_{\sigma}^{d} \in \tilde{H}_{n-2}\left(\bar{\Pi}_{n d}^{0 \bmod d} ; \mathbb{Z}\right)$. In each poset $\bar{\Pi}_{\sigma}^{d}$, we select a distinguished maximal chain $c_{\sigma}^{d}$ whose $k$ block partition is obtained by splitting $\sigma$ at positions $d, 2 d, \ldots,(k-1) d$, for $k=2,3, \ldots, n$. For example,

$$
c_{123456}^{2}=(12 / 34 / 56<12 / 3456) \text {. }
$$

Let

$$
A_{n}^{d}:=\left\{\sigma \in \mathfrak{S}_{n d}: \operatorname{des}(\sigma)=\{d, 2 d, \ldots,(n-1) d\}, \sigma(n d)=n d\right\} .
$$

Recall that in Exercise 4.3.6 (d), it is stated that $\bar{\Pi}_{n d}^{0 \bmod d}$ has the homotopy type of a wedge of $\left|A_{n}^{d}\right|$ spheres of dimension $n-2$.

Theorem 4.5.1 (Wachs $[\mathbf{1 9 6}])$. The set $\left\{\rho_{\sigma}^{d}: \sigma \in A_{n}^{d}\right\}$ forms a basis for $\tilde{H}_{n-2}\left(\bar{\Pi}_{n d}^{0 \bmod d} ; \mathbb{Z}\right)$ and the set $\left\{c_{\sigma}^{d}: \sigma \in A_{n}^{d}\right\}$ forms a basis for $\tilde{H}^{n-2}\left(\bar{\Pi}_{n d}^{0 \bmod d} ; \mathbb{Z}\right)$.

The theorem is proved by first showing that for all $\alpha, \beta \in A_{n}^{d}$, if $c_{\alpha}^{d} \in \Pi_{\beta}^{d}$ then $\alpha \leq \beta$ in lexicographic order. This is used to establish linear independence of both $\left\{\rho_{\sigma}^{\bar{d}}: \sigma \in A_{n}^{d}\right\}$ and $\left\{c_{\sigma}^{d}: \sigma \in A_{n}^{d}\right\}$. The result then follows from Exercise $4.3 .6(\mathrm{~d})$. 
The "splitting basis" given in Theorem 4.5.1 is used in [196] to give a combinatorial proof of the following result of Calderbank Hanlon and Robinson, which was first conjectured by Stanley.

Theorem 4.5.2 (Calderbank, Hanlon and Robinson [54]). Let $H_{n, d}$ be the skew hook of size $n d-1$ and descent set $\{d, 2 d, \ldots,(n-1) d\}$ (cf. Section 2.2). Then

$$
\tilde{H}_{n-2}\left(\bar{\Pi}_{n d}^{0 \bmod d}\right) \downarrow_{\mathfrak{S}_{n d-1}}^{\mathfrak{S}_{n d}} \cong \mathfrak{S}_{n d-1} S^{H_{n, d}}
$$

Calderbank, Hanlon, Robinson obtain this result as a consequence of (4.4.8). By mapping tableaux of shape $H_{n, d}$ to maximal chains of $\bar{\Pi}_{n d}^{0 \bmod d}$, one gets a combinatorial proof. Indeed, for each tableaux $T$ of shape $H_{n, d}$, let $\sigma_{T}$ be the permutation obtained by reading the entries of the skew hook tableaux $T$ from the southwest end to the northeast end of $T$ and then attaching $n d$. Now define

$$
c_{T}:=c_{\sigma_{T}}^{d} \text { and } \rho_{T}:=\rho_{\sigma_{T}}^{d} .
$$

Theorem 4.5.3 (Wachs [196]). The map $T \mapsto c_{T}$ induces a well-defined $\mathfrak{S}_{n d-1}$ isomorphism from the skew hook Specht module $S^{H_{n, d}}$ to $\tilde{H}^{n-2}\left(\bar{\Pi}_{n d}^{0 \bmod d}\right) \downarrow_{\mathfrak{S}_{n d-1} d}^{\mathfrak{S}_{n d}}$.

To prove this, one first observes that the row permutations leave $c_{T}$ invariant; then one shows that the Garnir relations map to cohomology relations.

There is a dual version of polytabloid defined for each tableaux $T$ by

$$
e_{T}^{*}:=\sum_{\alpha \in R_{\lambda}} \sum_{\beta \in C_{\lambda}} \operatorname{sgn}(\beta) T \beta \alpha .
$$

(This is actually closer to the traditional notion of polytabloid than the one we gave in Section 2.2.) For each skew or straight shape $\lambda$, it is known that

$$
\left\langle e_{T}^{*}: T \in \mathcal{T}_{\lambda}\right\rangle \cong \mathfrak{S}_{n} S^{\lambda} .
$$

Theorem 4.5.4 (Wachs $[\mathbf{1 9 6}]$ ). The map $e_{T}^{*} \mapsto \rho_{T}$ induces a well-defined $\mathfrak{S}_{n d-1^{-}}$ isomorphism from $S^{H_{n, d}}$ to $\tilde{H}_{n-2}\left(\bar{\Pi}_{n d}^{0 \bmod d}\right) \downarrow_{\mathfrak{S}_{n d-1}}^{\mathfrak{S}_{n d}}$.

All the results of this subsection were generalized to the restricted block size partition posets $\Pi_{n}^{S}$ of Theorem 4.4.11 by Browdy and Wachs [50, 51]. The nonpurity of $\Pi_{n}^{S}$ in the general case significantly increases the complexity of the results. The "at least $k$ " partition poset $\Pi_{\bar{n}}^{\geq k}$ is an example of such a nonpure poset.

\subsubsection{The $1 \bmod d$ partition lattice $\Pi_{n d+1}^{1} \bmod d$}

First we describe a basis for top cohomology of $\bar{\Pi}_{n d+1}^{1} \bmod d$, due to Hanlon and Wachs [91], which generalizes the decreasing chain basis $\left\{\bar{c}_{\sigma}: \sigma \in \mathfrak{S}_{n}, \sigma(n)=n\right\}$ for cohomology of $\bar{\Pi}_{n}$ given in Section 3.2.2. This basis is used in [91] to prove a generalization of Theorem 1.6.2, which relates the $\mathfrak{S}_{n d+1}$-module $\tilde{H}^{n-2}\left(\bar{\Pi}_{n d+1}^{1 \bmod d}\right)$ to a $(d+1)$-ary version of the free Lie algebra. Then we describe a basis for top homology found about ten years later by Shareshian and Wachs [157], which generalizes the tree-splitting basis for homology of $\bar{\Pi}_{n}$ given in Section 3.2.2. This basis is used in [157] to relate the $\mathfrak{S}_{n d+1}$-module $\tilde{H}^{n-2}\left(\bar{\Pi}_{n d+1}^{1 \bmod d}\right)$ to the homology of graph complexes studied by Linusson Shareshian and Welker [120] and Jonsson [103].

Let $\mathcal{T}_{n d+1}^{d}$ be the set of rooted planar $(d+1)$-ary trees on leaf set $[n d+1]$ (i.e., rooted trees in which each internal node has exactly $d+1$ children that are ordered from left to right). For any node $x$ of $T \in \mathcal{T}_{n d+1}^{d}$, let $m(x)$ be the smallest leaf in 


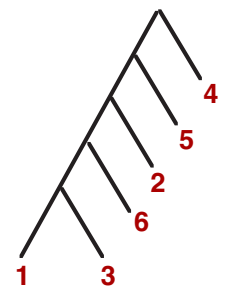

(a)

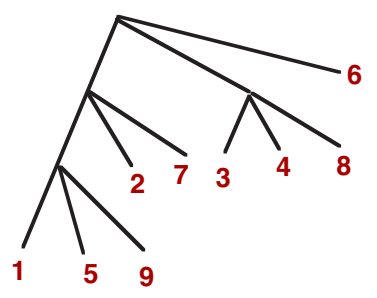

(b)

Figure 4.5.2

the tree rooted at $x$. A tree $T$ in $\mathcal{T}_{n d+1}^{d}$ is said to be a $d$-brush if for each node y of $T$, the $m$-values of the children of $y$ increase from left to right, and the child with the largest $m$-value is a leaf. An example of a 1-brush is given in Figure 4.5.2 (a) and of a 2-brush is given in Figure 4.5.2 (b). Note that every 1-brush looks like a comb, which is the reason for the terminology "brush".

\section{Exercise 4.5.5.}

(a) Show that the number of 1 -brushes on leaf set $[n+1]$ is $n$ !.

(b) Show that the number of 2 -brushes on leaf set $[2 n+1]$ is $(2 n-1) ! !^{2}$.

Recall from Section 1.6 that the postorder traversal of a binary tree on leaf set $[n]$ yields a maximal chain of $\bar{\Pi}_{n}$. Now we consider a more general construction, which associates a maximal chain $c_{T}$ of $\bar{\Pi}_{n d+1}^{1} \bmod d$ to each tree $T$ in $\mathcal{T}_{n d+1}^{d}$. Each internal node $y$ of $T$ corresponds to a merge of $d+1$ blocks that are the leaf sets of the trees rooted at the $d+1$ children of $y$. Postorder traversal of internal nodes of $T$ yields a sequence of merges, which corresponds to a maximal chain $c_{T}$ of $\bar{\Pi}_{n d+1}^{1 \bmod d}$. For example if $T$ is the tree of Figure 4.5.2 (b) then

$$
c_{T}=159 / 2 / 7 / 3 / 4 / 8 / 6<15927 / 3 / 4 / 8 / 6<15927 / 348 / 6
$$

Theorem 4.5.6 (Hanlon and Wachs $[\mathbf{9 1}]$ ). Let $\mathcal{B}_{n d+1}^{d}$ be the set of $d$-brushes in $\mathcal{T}_{n d+1}^{d}$. The set $\left\{c_{T}: T \in \mathcal{B}_{n d+1}^{d}\right\}$ forms a basis for $\tilde{H}^{n-2}\left(\bar{\Pi}_{n d+1}^{1 \bmod d} ; \mathbb{Z}\right)$.

Exercise 4.5.7 (Hanlon and Wachs [91]). Prove Theorem 4.5.6 by showing

(a) The set $\left\{c_{T}: T \in \mathcal{B}_{n d+1}^{d}\right\}$ spans $\tilde{H}^{n-2}\left(\bar{\Pi}_{n d+1}^{1 \bmod d} ; \mathbb{Z}\right)$.

(b) $\left|\mathcal{B}_{n d+1}^{d}\right|=\left|\mu\left(\Pi_{n d+1}^{1 \bmod d}\right)\right|$.

We now construct the Shareshian-Wachs basis for homology of $\bar{\Pi}_{n d+1}^{1 \bmod d}$. Just like the tree-splitting basis, which it generalizes, it consists of fundamental cycles of Boolean algebras embedded in $\Pi_{n d+1}^{1} \bmod d$.

A connected graph $G$ is said to be a $d$-clique tree if either $G$ consists of a single node, or $G$ contains a $d$-clique, the removal of whose edges results in a graph with $d$ connected components that are all $d$-clique trees. Note that each edge of a $d$-clique tree is in a unique $d$-clique and the removal of the edges of any $d$-clique from a $d$-clique tree results in a graph with $d$ connected components that are all $d$-clique trees. Note also that a 2-clique tree is an ordinary tree. An example of a 3-clique tree, which we refer to as a triangle tree, is given in Figure 4.5.3. 


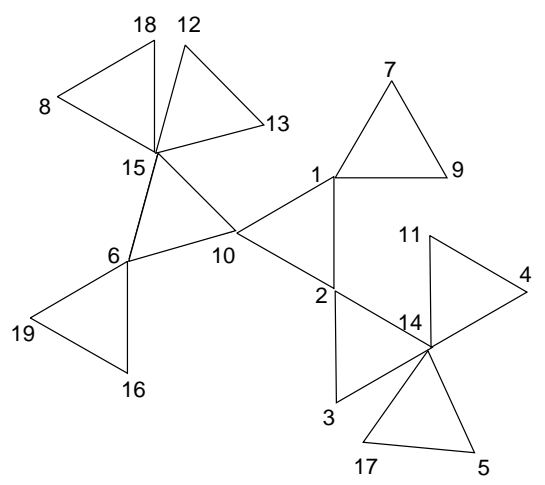

Figure 4.5.3

Given a $(d+1)$-clique tree $T$ on node set $[n d+1]$, one obtains a partition in $\Pi_{n d+1}^{1 \bmod d}$ by choosing any set of $(d+1)$-cliques of $T$ and removing the edges of each clique in the set. The blocks of the partition are the node sets of the connected components of the resulting graph. We say that the partition is obtained by splitting the $(d+1)$-clique tree $T$ at the chosen set of $(d+1)$-cliques. For example, the partition obtained by splitting the triangle tree at the shaded triangles in Figure 4.5.4 is

$$
19,16,6 / 8,15,18,12,13 / 10,2,1,9,7 / 3 / 17,5,4,11,14
$$

Now let $\Pi_{T}$ be the subposet of $\Pi_{n d+1}^{1} \bmod d$ consisting of partitions obtained by splitting $T$. Clearly $\Pi_{T}$ is isomorphic to the subset lattice $B_{n}$. Therefore $\Delta\left(\bar{\Pi}_{T}\right)$ is an $(n-2)$ sphere which determines a fundamental cycle $\rho_{T}$.

Theorem 4.5.8 (Shareshian and Wachs [157]). The set of fundamental cycles $\rho_{T}$ such that $T$ is a $(d+1)$-clique tree on node set $[n d+1]$, spans $\tilde{H}_{n-2}\left(\bar{\Pi}_{n d+1}^{1} \bmod d ; \mathbb{Z}\right)$.

This is proved by identifying a set $S$ of $(d+1)$-clique trees, called increasing $(d+$ 1)-clique trees, and establishing a bijection and unitriangular relationship between $(d+1)$-clique trees and $d$-brushes. This shows that $\left\{\rho_{T}: T \in S\right\}$ is a basis for $\tilde{H}_{n-2}\left(\bar{\Pi}_{n d+1}^{1 \bmod d} ; \mathbb{Z}\right)$. The increasing 2 -clique trees are the increasing trees discussed in Section 3.2.2 and the homology basis is the tree splitting basis.

Next we discuss an application of Theorem 4.5.8 that led to the discovery of the clique tree splitting basis in the first place. Let $\mathrm{NPM}_{2 n}$ be the poset of nonempty graphs on node set $[2 n]$ that don't contain a perfect matching (i.e., a subgraph in which each of the $2 n$ vertices has degree 1 ), ordered by inclusion of edge sets. Linusson, Shareshian, and Welker [120] show, using discrete Morse theory, that $\mathrm{NPM}_{2 n}$ has the homotopy type of a wedge of $(2 n-1) ! !^{2}$ spheres of dimension $3 n-4$. It was in this work that the increasing triangle trees first arose as the critical elements of a Morse matching on $\mathrm{NPM}_{2 n}$ (increasing triangle trees are just called trees of triangles in $[\mathbf{1 2 0}]$ ). See the chapter by Forman [74] in this volume to 


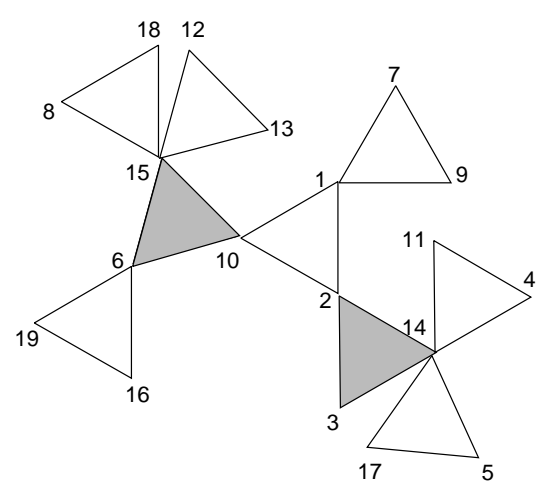

Figure 4.5.4

learn about discrete Morse theory and critical elements. Recall that the property of containing (or not containing) a perfect matching is an example of a monotone graph property (see Section 1.4). When we discuss Alexander duality in the next lecture, we will see how the homology of the poset of graphs that have a monotone graph property is related to the homology of the poset of graphs that don't have the property.

Theorem 4.5.8 and discrete Morse theory play essential roles in the proof of the following equivariant version of the Linusson-Shareshian-Welker result. Indeed, discrete Morse theory is used to show that there is a sign twisted $\mathfrak{S}_{2 n-1}$-isomorphism between the homology of $\mathrm{NPM}_{2 n}$ and the cohomology of another poset called the factor critical graph poset. Discrete Morse theory is also used to show that the cohomology of the factor critical graph poset is generated by certain maximal chains naturally indexed by triangle trees. This gives a natural map from the triangle tree generators $\rho_{T}$ of homology of $\Pi_{2 n-1}^{1} \bmod 2$ to the maximal chains of the factor critical graph poset that are indexed by the triangle trees. Relations on the triangle tree generators of $\tilde{H}_{n-3}\left(\bar{\Pi}_{2 n-1}^{1} \bmod 2\right)$ are derived, which are shown to map to coboundary relations in the factor critical graph poset.

Theorem 4.5.9 (Shareshian and Wachs [157]). For all $n \geq 1$,

$$
\tilde{H}_{3 n-4}\left(\mathrm{NPM}_{2 n}\right) \downarrow_{\mathfrak{S}_{2 n-1}}^{\mathfrak{S}_{2 n}} \cong_{\mathfrak{S}_{2 n-1}} \tilde{H}_{n-3}\left(\bar{\Pi}_{2 n-1}^{1 \bmod 2}\right) \otimes \operatorname{sgn}_{2 n-1}
$$

Another graph poset, recently studied by Jonsson [103], curiously has the same bottom nonvanishing homology as $\mathrm{NPM}_{2 n}$ and $\bar{\Pi}_{2 n-1}^{1 \bmod 2}$ (up to sign twists). A graph is said to be $d$-edge-connected if removal of any set of at most $d-1$ edges leaves the graph connected. So a 1-edge-connected graph is just a connected graph. The $d$-clique trees are examples of graphs that are $(d-1)$-edge-connected but not $d$ edge-connected. In fact, the $d$-clique trees are minimal elements of the poset of $(d-1)$-edge-connected graphs. Jonsson studied the integral homology of the poset of graphs that are not 2-edge-connected. Let $\mathrm{NEC}_{n}^{d}$ be the poset of nonempty graphs on node set $[n]$ that are not $d$-edge-connected. Jonsson discovered that 
$\mathrm{NEC}_{2 n+1}^{2}$ has homology in multiple dimensions and that the bottom nonvanishing reduced integral homology is in dimension $3 n-2$ and is of rank $(2 n-1) ! !^{2}$. His proof involves discrete Morse theory with the increasing triangle trees as the critical elements. Shareshian and Wachs $[\mathbf{1 5 7}]$ obtain the following equivariant version of Jonsson's result by giving presentations of the two homology groups in terms of triangle-trees.

Theorem 4.5.10 (Shareshian and Wachs $[\mathbf{1 5 7}]$ ). For all $n$,

$$
\tilde{H}_{3 n-2}\left(\mathrm{NEC}_{2 n+1}^{2}\right) \cong_{\mathfrak{S}_{2 n+1}} \tilde{H}_{n-2}\left(\bar{\Pi}_{2 n+1}^{1 \bmod 2}\right) \otimes \operatorname{sgn}_{2 n+1} .
$$

Conjecture 4.5.11 (Shareshian and Wachs $[\mathbf{1 5 7}]$ ). For all $n, d \geq 1$,

$$
\tilde{H}_{\left(\begin{array}{c}
d+1 \\
2
\end{array}\right) n-2}\left(\mathrm{NEC}_{d n+1}^{d}\right) \cong_{\mathfrak{S}_{d n+1}} \tilde{H}_{n-2}\left(\bar{\Pi}_{d n+1}^{1 \bmod d}\right) \otimes \operatorname{sgn}_{d n+1}^{\otimes d+1},
$$

and

$$
\tilde{H}_{i}\left(\mathrm{NEC}_{d n+1}^{d}\right)=0
$$

if $i<\left(\begin{array}{c}d+1 \\ 2\end{array}\right) n-2$.

The conjecture is true for $d=1,2$. Indeed, Jonsson's homology result and Theorem 4.5.10 comprise the $d=2$ case. The conjecture for $d=1$ says that that the poset of disconnected graphs on node set $[n+1]$ has homology isomorphic, as an $\mathfrak{S}_{n+1}$-module, to that of the partition lattice. A proof of this well-known result is discussed in Example 5.2.8.

There is another interesting poset with the same homotopy type and $\mathfrak{S}_{n d+1^{-}}$ equivariant homology as that of $\bar{\Pi}_{n d+1}^{1 \bmod d}$, worth mentioning here. This poset is the proper part of the poset $\mathcal{T}_{n d+2}^{2 \text { mod } d}$ of homeomorphically irreducible trees on leaf set $[n d+2]$ in which each internal node has degree congruent to $2 \bmod d$. By homeomorphically irreducible we mean nonrooted and no node has degree 2 . The order relation is as follows: $T_{1}<T_{2}$ if $T_{1}$ can be obtained from $T_{2}$ by contracting internal edges. So the bottom element of $\mathcal{T}_{n d+2}^{2 \bmod d}$ is the star tree (the tree with only one internal node), and the maximal elements are trees in which each internal node has degree exactly $d+2$. The $d=1$ case of the tree poset $\mathcal{T}_{n d+2}^{2 \bmod d}$ has arisen in various areas such as algebraic geometry, homotopy theory, geometric group theory, mathematical physics and mathematical biology; see eg., [46, 195, 146, 209, 145, $\mathbf{1}, \mathbf{1 8}, \mathbf{1 3 6}]$ and the references contained therein. Vogtmann $[\mathbf{1 9 5}]$ showed that the tree poset in the $d=1$ case is homotopy Cohen-Macaulay. There is a natural action of $\mathfrak{S}_{n d+2}$ on $\mathcal{T}_{n d+2}^{2 \bmod d}$ whose representation on the homology of $\mathcal{T}_{n d+2}^{2 \bmod d}-\{\hat{0}\}$ was computed by Robinson and Whitehouse $[\mathbf{1 4 6}]$ in the $d=1$ case. Hanlon $[\mathbf{8 7}]$ introduced the general tree poset $\mathcal{T}_{n d+2}^{2}$ mod $d$, proved that it is Cohen-Macaulay, and generalized the Robinson-Whitehouse result.

Theorem 4.5.12 (Robinson and Whitehouse, $d=1$ [146], Hanlon [87]). For all $d, n \geq 1$,

$$
\tilde{H}_{n-2}\left(\overline{\mathcal{T}}_{n d+2}^{2 \bmod d}\right) \cong \cong_{\mathfrak{S}_{n d+2}} \quad \tilde{H}_{n-2}\left(\bar{\Pi}_{n d+1}^{1 \bmod d}\right) \uparrow_{\mathfrak{S}_{n d+1}}^{\mathfrak{S}_{n d+2}}-\tilde{H}_{n-1}\left(\bar{\Pi}_{n d+2}^{1 \bmod d}\right) .
$$

The $d=1$ case of the $\mathfrak{S}_{n d+2}$-module given in Theorem 4.5.12 has come to be known as the Whitehouse module. It has occurred in a variety of diverse contexts such as homotopy theory $[\mathbf{1 4 6}, \mathbf{2 0 9}]$, cyclic Lie operads $[\mathbf{7 9}, \mathbf{1 0 9}]$, homology of partition posets $[\mathbf{1 7 9}, \mathbf{1 8 0}]$, knot theory and graph complexes $[\mathbf{8}]$, and hyperplane arrangements and Lie algebra homology [90]. 
From Theorem 4.5.12, one can show that (see [181]),

$$
\tilde{H}_{n-2}\left(\overline{\mathcal{T}}_{n d+2}^{2 \bmod d}\right) \downarrow_{\mathfrak{S}_{n d+1}}^{\mathfrak{S}_{n d+2}} \cong \mathfrak{S}_{n d+1} \quad \tilde{H}_{n-2}\left(\bar{\Pi}_{n d+1}^{1 \bmod d}\right) .
$$

A direct combinatorial proof of this is discussed in the following exercise.

Exercise 4.5.13. By removing the leaf $n d+2$ from any tree $T$ in $\mathcal{T}_{n d+2}^{2 \bmod d}$ and designating the internal node that had been adjacent to the leaf $n d+2$ as the root, one turns $T$ into a rooted nonplanar tree on leaf set $[n d+1]$ in which each internal node has $i d+1$ children, for some $i \geq 1$. The maximal elements of $\mathcal{T}_{n d+2}^{2 \text { mod } d}$ are now rooted nonplanar $(d+1)$-ary trees on leaf set $[n d+1]$.

(a) Show that any linear extension of the following partial ordering of rooted nonplanar $(d+1)$-ary trees on leaf set $[n d+1]$ is a recursive coatom ordering of $\mathcal{T}_{n d+2}^{2 \bmod d} \cup\{\hat{1}\}$. Let $T_{1} \wedge T_{2} \wedge \cdots \wedge T_{d+1}$ denote the $(d+1)$-tree in which $T_{1}, T_{2}, \ldots, T_{d+1}$ are the subtrees of the root. The partial order is defined to be the transitive closure of the relation given by $T<T^{\prime}$ if $T^{\prime}$ can be obtained from $T$ by replacing some subtree

$$
\left(T_{1} \wedge \cdots \wedge T_{d+1}\right) \wedge T_{d+2} \wedge \cdots \wedge T_{2 d+1}
$$

with the subtree

$$
\left(T_{1} \wedge \cdots \wedge T_{d} \wedge T_{d+2}\right) \wedge T_{d+1} \wedge T_{d+3} \wedge T_{2 d+1}
$$

where the minimum leaf of $T_{d+2}$ is less than the minimum leaf of $T_{d+1}$.

(b) The poset $\mathcal{T}_{n d+2}^{2 \bmod d}$ can be viewed as the face poset of a simplicial complex whose facets correspond to rooted nonplanar $(d+1)$-ary trees on leaf set $[n d+1]$. Hence the recursive coatom ordering of (a) is simply a shelling of the simplicial complex. Show that the homology facets of the shelling correspond to the $d$-brushes. Consequently,

$$
\overline{\mathcal{T}}_{n d+2}^{2 \bmod d} \simeq \bar{\Pi}_{n d+1}^{1 \bmod d} .
$$

(c) Prove (4.5.1) by first observing that the set of the rooted planar $(d+1)$ ary trees on leaf set $[n d+1]$ indexes respective sets of maximal chains of $\bar{\Pi}_{n d+1}^{1 \bmod d}$ and $\overline{\mathcal{T}}_{n d+2}^{2 \bmod d}$ that generate top cohomology, and then showing that the two cohomology groups have the same presentation in terms of rooted planar $(d+1)$-ary trees on leaf set $[n d+1]$.

Trappmann and Ziegler [188] established shellability of $\mathcal{T}_{n d+2}^{2 \bmod d}$ in a different way from Exercise 4.5.13 (a), but with the same homology facets as in Exercise 4.5.13 (b). Ardila and Klivans [1] and Robinson [145] have recently independently proved a result stronger than the homotopy result (4.5.2) and the homology result (4.5.1) in the $d=1$ case; namely that $\overline{\mathcal{T}}_{n+1}^{2 \bmod 1}$ and $\bar{\Pi}_{n}$ are $\mathfrak{S}_{n}$-homeomorphic. Robinson's homeomorphism for the $d=1$ case restricts to a homeomorphism for the general case. Hence $\overline{\mathcal{T}}_{n d+2}^{2 \bmod d}$ and $\bar{\Pi}_{n d+1}^{1 \bmod d}$ are $\mathfrak{S}_{n d+1}$-homeomorphic for all $n, d \geq 1$.

Problem 4.5.14. It is well-known that for each permutation in $\mathfrak{S}_{n}$, there is a distinct copy of the face poset of the $(n-1)$-dimensional associahedron embedded in $\mathcal{T}_{n+2}^{2 \bmod 1}$ and that by taking fundamental cycles, one obtains a basis for the homology of $\overline{\mathcal{T}}_{n+2}^{2 \bmod 1}$. The associahedron is discussed in the chapter of Fomin and Reading in this volumn [71]. Is there a nice basis for homology of $\overline{\mathcal{T}}_{n d+2}^{2 \bmod d}$ for general $d$, consisting of fundamental cycles of embedded face posets of polytopes, which are copies of some sort of $d$-analog of the associahedron? 
By Theorem 4.5.9 and the isomorphism (4.5.1), the restriction of the $\mathfrak{S}_{2 n^{-}}$ equivariant homology of $\mathrm{NPM}_{2 n}$ and $\overline{\mathcal{T}}_{2 n}^{2 \bmod 2}$ to $\mathfrak{S}_{2 n-1}$ are isomorphic (up to tensoring with the sign representation). For the unrestricted homology modules, we have the following conjecture.

Conjecture 4.5.15 (Linusson, Shareshian and Welker [120]). For all $n \geq 1$,

$$
\tilde{H}_{3 n-4}\left(\mathrm{NPM}_{2 n}\right) \cong \mathfrak{S}_{2 n} \tilde{H}_{n-4}\left(\overline{\mathcal{T}}_{2 n}^{2 \bmod 2}\right) \otimes \operatorname{sgn}_{2 n} \text {. }
$$

\subsection{Fixed point Möbius invariant}

We conclude this lecture with a very brief discussion of another commonly used recursive technique for computing homology representations. Let $P$ be a $G$-poset and for each $g \in G$, let $P^{g}$ denote the induced subposet of $P$ consisting of elements that are fixed by $g$. Let $\chi^{P}$ be the character of the virtual representation of $G$ on $\oplus_{i}(-1)^{i} \tilde{H}_{i}(P)$. The following consequence of the Hopf-Lefschetz fixed point theorem first appeared in the combinatorics literature in the work of Baclawski and Björner [13].

Theorem 4.6.1. For any $G$-poset $P$ and $g \in G$,

$$
\chi^{P}(g)=\mu\left(\hat{P}^{g}\right) .
$$

There are many interesting applications of this theorem in the literature. We give a simple example here.

Example 4.6.2 (Stanley, see [25]). Consider the normal word poset $\mathcal{N}_{n, k}$ under the action of $\mathfrak{S}_{n}$. Since $P:=\overline{\mathcal{N}}_{n, k}$ is Cohen-Macaulay, $(-1)^{k-1} \chi^{P}$ is the character of the representation of $\mathfrak{S}_{n}$ on $\tilde{H}_{k-1}\left(\overline{\mathcal{N}}_{n, k}\right)$. The words that are fixed by $g$ are the words whose letters are fixed points of $g$. So $P^{g}$ is the poset of normal nonempty words of length at most $k$ on alphabet $F(g)$, where $F(g)$ is the set of fixed points of $g$. By Exercise 4.3.2 (c), $\mu\left(\hat{P}^{g}\right)=(-1)^{k-1}(|F(g)|-1)^{k}$. Since the irreducible representation $S^{(n-1,1)}$ has the character $(|F(g)|-1)$, its $k$ th tensor power has the character $(|F(g)|-1)^{k}$. By Theorem 4.6.1,

$$
\tilde{H}_{k-1}\left(\overline{\mathcal{N}}_{n, k}\right) \cong \mathfrak{S}_{n}\left(S^{(n-1,1)}\right)^{\otimes k} \text {. }
$$

Since the identical argument works for $\mathcal{W}_{n, k}$,

$$
\tilde{H}_{k-1}\left(\overline{\mathcal{W}}_{n, k}\right) \cong \mathfrak{S}_{n}\left(S^{(n-1,1)}\right)^{\otimes k} .
$$

Exercise 4.6.3. Show that if $\chi$ is the character of the representation of $\mathfrak{S}_{n}$ on the homology of the injective word poset $\overline{\mathcal{I}}_{n, n}$ then

$$
\chi(g)=d_{|F(g)|},
$$

where $d_{j}$ is the number of derangements of $j$ letters. 


\section{LECTURE 5 \\ Poset operations and maps}

\subsection{Operations: Alexander duality and direct product}

In this section we consider some fundamental operations on posets and their affect on homology. The operations are Alexander duality, join and direct product.

Theorem 5.1.1 (Poset Alexander duality, Stanley $[\mathbf{1 6 7 , ~ 1 6 9 ] ) . ~ L e t ~} P$ be a $G$-poset whose order complex triangulates an $n$-sphere. If $Q$ is any induced $G$-subposet of $P$ then for all $i$,

$$
\tilde{H}_{i}(Q) \cong_{G} \tilde{H}^{n-i-1}(P-Q) \otimes \tilde{H}_{n}(P) .
$$

If $Q$ is any induced subposet of $P$ then for all $i$,

$$
\tilde{H}_{i}(Q ; \mathbb{Z}) \cong \tilde{H}^{n-i-1}(P-Q, \mathbb{Z}) .
$$

Exercise 5.1.2. Let $P$ be the poset of all graphs on node set $[n]$ ordered by inclusion of edge sets. The symmetric group $\mathfrak{S}_{n}$ acts on $P$ in the obvious way, making $P$ an $\mathfrak{S}_{n}$-poset. Let $Q$ be an induced subposet of $P$ invariant under the action of $\mathfrak{S}_{n}$. Show that for all $i$,

$$
\tilde{H}_{i}(\bar{Q}) \cong \mathfrak{S}_{n} \tilde{H}_{\left(\begin{array}{c}
n \\
2
\end{array}\right)-i-3}(\bar{P}-\bar{Q}) \otimes \operatorname{sgn}_{n}^{\otimes n}
$$

For example, if $Q$ is the poset of connected graphs on node set $[n]$ and $R$ is the poset of disconnected graphs on node set $[n]$ then

$$
\tilde{H}_{i}(\bar{Q}) \cong_{\mathfrak{S}_{n}} \tilde{H}_{\left(\begin{array}{c}
n \\
2
\end{array}\right)-i-3}(\bar{R}) \otimes \operatorname{sgn}_{n}^{\otimes n}
$$

Exercise 5.1.3. Given a simplicial complex $\Delta$ on vertex set $V$, its Alexander dual $\Delta^{\vee}$ is defined to be the simplicial complex on $V$ consisting of complements of nonfaces of $\Delta$, i.e.,

$$
\Delta^{\vee}=\{V-F: F \subseteq V \text { and } F \notin \Delta\}
$$

How are the homology of $\Delta$ and its Alexander dual related?

Let $P$ be a $G$-poset and let $Q$ be an $H$-poset. Then the join $P * Q$ and the product $P \times Q$ are $(G \times H)$-posets with respective $(G \times H)$ actions given by

$$
(g, h) x=\left\{\begin{array}{l}
g x \text { if } x \in P \\
h x \text { if } x \in Q
\end{array},\right.
$$


and

$$
(g, h)(p, q)=(g p, h q) .
$$

We have the following poset version of the Künneth theorem of algebraic topology.

Theorem 5.1.4. Let $P$ be a $G$ poset and let $Q$ be an $H$-poset. Then

$$
\tilde{H}_{r}(P * Q) \cong_{G \times H} \bigoplus_{i} \tilde{H}_{i}(P) \otimes \tilde{H}_{r-i-1}(Q),
$$

for all $r$.

A result of Quillen [135] states that if $P$ and $Q$ have bottom elements $\hat{0}_{P}$ and $\hat{0}_{Q}$, respectively, then there is a $(G \times H)$-homeomorphism

$$
P \times Q \backslash\left\{\left(\hat{0}_{P}, \hat{0}_{Q}\right)\right\} \cong_{G \times H} P \backslash\left\{\hat{0}_{P}\right\} * Q \backslash\left\{\hat{0}_{Q}\right\} .
$$

Walker $[\mathbf{2 0 4}, \mathbf{2 0 5}$ ] proves the similar result that if $P$ and $Q$ have top elements as well as bottom elements then there is a $(G \times H)$-homeomorphism

$$
\overline{P \times Q} \cong_{G \times H} \bar{P} * \bar{Q} * A_{2},
$$

where $A_{2}$ is a two element antichain on which the trivial group acts. Theorem 5.1.4 and Quillen's and Walker's results yield,

Theorem 5.1.5. Let $P$ be a G-poset with a bottom element $\hat{0}_{P}$ and let $Q$ be an $H$-poset with a bottom element $\hat{0}_{Q}$. Then for all $r$,

$$
\tilde{H}_{r}\left(P \times Q \backslash\left\{\left(\hat{0}_{P}, \hat{0}_{Q}\right)\right\} \cong_{G \times H} \bigoplus_{i} \tilde{H}_{i}\left(P \backslash\left\{\hat{0}_{P}\right\}\right) \otimes \tilde{H}_{r-i-1}\left(Q \backslash\left\{\hat{0}_{Q}\right\}\right) .\right.
$$

If $P$ and $Q$ also have top elements then for all $r$,

$$
\tilde{H}_{r}(\overline{P \times Q}) \cong_{G \times H} \bigoplus_{i} \tilde{H}_{i}(\bar{P}) \otimes \tilde{H}_{r-i-2}(\bar{Q}) .
$$

Exercise 5.1.6. Let $P$ and $Q$ be posets with bottom elements. Show that $P \times Q$ is Cohen-Macaulay if and only if $P$ and $Q$ are Cohen-Macaulay. This result also holds in the nonpure case but is more difficult to prove; see [44].

The products in Theorem 5.1.5 are known as reduced products. There is a similar formula for the homology of ordinary direct products which follows from the Künneth theorem and another $(G \times H)$-homeomorphism

$$
P \times Q \cong\|P\| \times\|Q\|
$$

of Quillen [135] and Walker [205].

Theorem 5.1.7. Let $P$ be a $G$-poset and let $Q$ be an $H$ poset. Then for all $r$,

$$
H_{r}(P \times Q) \cong_{G \times H} \bigoplus_{i} H_{i}(P) \otimes H_{r-i}(Q) .
$$

Next we consider the $n$-fold product $P^{\times n}$ of a $G$-poset $P$. The group that acts on $P^{\times n}$ is the wreath product $\mathfrak{S}_{n}[G]$, defined in Section 2.4. The action on $P^{\times n}$ is given by

$$
\left(g_{1}, g_{2}, \ldots, g_{n} ; \sigma\right)\left(p_{1}, p_{2}, \ldots, p_{n}\right)=\left(g_{1} p_{\sigma^{-1}(1)}, g_{2} p_{\sigma^{-1}(2)}, \ldots, g_{n} p_{\sigma^{-1}(n)}\right) .
$$


For each pair of positive integers $i$ and $m$, define the $\mathfrak{S}_{m}[G]$-module

$$
H(P, i, m):= \begin{cases}S^{(m)}\left[H_{i-1}(P)\right] & \text { if } i \text { is odd } \\ S^{\left(1^{m}\right)}\left[H_{i-1}(P)\right] & \text { if } i \text { is even. }\end{cases}
$$

For a partition $\lambda$ with $m_{i}$ parts equal to $i$ for each $i$, let

$$
\mathfrak{S}_{\lambda}[G]:=\underset{i: m_{i}>0}{\mathbf{X}} \mathfrak{S}_{m_{i}}[G]
$$

and define the $\mathfrak{S}_{\lambda}[G]$-module

$$
H(P, \lambda):=\bigotimes_{i: m_{i}>0} H\left(P, i, m_{i}\right) .
$$

We can now state an analog of Theorem 5.1.7.

Theorem 5.1.8 (Sundaram and Welker $[\mathbf{1 8 3}]$ ). Let $P$ be a $G$-poset. Then for all $r$,

$$
H_{r}\left(P^{\times n}\right) \cong_{\mathfrak{S}_{n}[G]} \bigoplus_{\substack{\lambda \vdash r+n \\ l(\lambda)=n}} H(P, \lambda) \uparrow_{\mathfrak{S}_{\lambda}[G]}^{\mathfrak{S}_{n}[G]} .
$$

For an analog of Theorem 5.1.5, define the $\mathfrak{S}_{m}[G]$-modules

$$
\tilde{H}(P, i, m, 1):= \begin{cases}S^{(m)}\left[\tilde{H}_{i-2}(P)\right] & \text { if } i \text { is odd } \\ S^{\left(1^{m}\right)}\left[\tilde{H}_{i-2}(P)\right] & \text { if } i \text { is even }\end{cases}
$$

and

$$
\tilde{H}(P, i, m, 2):= \begin{cases}S^{\left(1^{m}\right)}\left[\tilde{H}_{i-2}(P)\right] & \text { if } i \text { is odd } \\ S^{(m)}\left[\tilde{H}_{i-2}(P)\right] & \text { if } i \text { is even }\end{cases}
$$

and the $\mathfrak{S}_{\lambda}[G]$-modules

$$
\tilde{H}(P, \lambda, 1):=\bigotimes_{i: m_{i}>0} \tilde{H}\left(P, i, m_{i}, 1\right)
$$

and

$$
\tilde{H}(P, \lambda, 2):=\bigotimes_{i: m_{i}>0} \tilde{H}\left(P, i, m_{i}, 2\right) .
$$

Theorem 5.1.9 (Sundaram and Welker $[\mathbf{1 8 3}]$ ). Let $P$ be a $G$-poset with a bottom element $\hat{0}$. Then for all $r$,

$$
\tilde{H}_{r}\left(P^{\times n}-\{(\hat{0}, \ldots, \hat{0})\}\right) \cong \mathfrak{S}_{n}[G] \bigoplus_{\substack{\lambda \vdash r+n+1 \\ l(\lambda)=n}} \tilde{H}(P-\{\hat{0}\}, \lambda, 1) \uparrow \mathfrak{S}_{\mathfrak{S}_{\lambda}[G]}[G] .
$$

If $P$ also has a top element then for all $r$,

$$
\tilde{H}_{r}\left(\overline{P^{\times n}}\right) \cong \mathfrak{S}_{n}[G] \bigoplus_{\substack{\lambda \vdash r+2 \\ l(\lambda)=n}} \tilde{H}(\bar{P}, \lambda, 2) \uparrow \uparrow_{\mathfrak{S}_{\lambda}[G]}^{\mathfrak{S}_{n}[G]} .
$$

We remark that we have stated Theorems 5.1.8 and 5.1.9 in a form different from the original given in $[\mathbf{1 8 3}]$ but completely equivalent. 
Example 5.1.10 (The Boolean algebra $B_{n}$ ). We apply the second part of Theorem 5.1.9 to $B_{n}=B_{1}^{\times n}$. The trivial group $\mathfrak{S}_{1}$ acts on $B_{1}$ and this induces an action of $\mathfrak{S}_{n}\left[\mathfrak{S}_{1}\right]=\mathfrak{S}_{n}$ on $B_{n}$, which is precisely the action given in (2.3.1). Clearly $\tilde{H}_{i-2}\left(\bar{B}_{1}\right)=0$ unless $i=1$, in which case $H_{-1}\left(\bar{B}_{1}\right)$ is the trivial representation of $S_{1}$. It follows that

$$
\tilde{H}\left(B_{1}, 1, m, 2\right)=S^{\left(1^{m}\right)}\left[S^{(1)}\right]=S^{\left(1^{m}\right)},
$$

and $\tilde{H}\left(B_{1}, i, m, 2\right)=0$ for $i \neq 1$. Hence

$$
\tilde{H}\left(B_{1}, \lambda, 2\right)= \begin{cases}S^{\left(1^{n}\right)} & \text { if } \lambda=\left(1^{n}\right) \\ 0 & \text { otherwise. }\end{cases}
$$

It follows that the only nonzero term in the decomposition given in Theorem 5.1.9 is the term corresponding to $\lambda=\left(1^{n}\right)$ and $r=n-2$. Hence we recover the fact that $\tilde{H}_{r}\left(\bar{B}_{n}\right)$ is the sign representation for $r=n-2$ and is 0 otherwise.

Exercise 5.1.11. The face lattice $C_{n}$ of the $n$-cross-polytope with its top element removed can be expressed as a product,

$$
C_{n} \backslash \hat{1}=\left(C_{1} \backslash \hat{1}\right)^{\times n} .
$$

Since $\mathfrak{S}_{2}$ acts on $C_{1} \backslash\{\hat{1}\}$, the product induces an action of the hyperoctahedral group $\mathfrak{S}_{n}\left[\mathfrak{S}_{2}\right]$ on $C_{n} \backslash \hat{1}$.

(a) Show that this action is the action given in Example 2.3.3.

(b) Use Theorem 5.1.9 to prove that

$$
\tilde{H}_{r}\left(\bar{C}_{n}\right) \cong_{\mathfrak{S}_{n}\left[\mathfrak{S}_{2}\right]} \begin{cases}S^{\left(1^{n}\right)}\left[S^{\left(1^{2}\right)}\right] & \text { if } r=n-1 \\ 0 & \text { otherwise. }\end{cases}
$$

(c) Use Theorem 5.1.9 to obtain a generalization of the formula in (b) for the poset $X_{m}^{\times n}$, where $X_{m}$ is the poset consisting of a bottom element $\hat{0}$ and $m$ atoms.

Example 5.1.12 ([183]). In this example we compute the homology of lower intervals in the partition lattice $\Pi_{n}$. Each lower interval is isomorphic to a product of smaller partition lattices,

$$
[\hat{0}, x] \cong \underset{i}{\mathrm{X}} \Pi_{i}^{\times m_{i}}
$$

where $m_{i}$ is equal to the number of blocks of $x$ of size $i$. The stabilizer $\left(\mathfrak{S}_{n}\right)_{x}$ of $x$ under the action of $\mathfrak{S}_{n}$ on $\Pi_{n}$ is isomorphic to $\mathbf{X}_{i} \mathfrak{S}_{m_{i}}\left[\mathfrak{S}_{i}\right]$. The $\left(\mathfrak{S}_{n}\right)_{x}$-poset $[\hat{0}, x]$ is isomorphic to the $\mathbf{X}_{i} \mathfrak{S}_{m_{i}}\left[\mathfrak{S}_{i}\right]$-poset $\mathbf{X}_{i} \Pi_{i}^{\times m_{i}}$. We use the second parts of Theorems 5.1.5 and 5.1.9 to compute the representation of $\mathbf{X}_{i} \mathfrak{S}_{m_{i}}\left[\mathfrak{S}_{i}\right]$ on the homology of $(\hat{0}, x)$. This yields the following formula of Lehrer and Solomon [117] for the only nonvanishing homology,

$\tilde{H}_{r(x)-2}(\hat{0}, x) \cong \mathrm{X}_{i} \mathfrak{S}_{m_{i}}\left[\mathfrak{S}_{i}\right] \quad S^{\left(m_{1}\right)}\left[\tilde{H}_{-2}\left(\bar{\Pi}_{1}\right)\right] \otimes S^{\left(1^{m_{2}}\right)}\left[\tilde{H}_{-1}\left(\bar{\Pi}_{2}\right)\right] \otimes S^{\left(m_{3}\right)}\left[\tilde{H}_{0}\left(\bar{\Pi}_{3}\right)\right] \otimes \cdots$. By summing over all set partitions of rank $r$ and taking Frobenius characteristic one gets,

ch $\bigoplus_{\substack{x \in \Pi_{n} \\ r(x)=r}} \tilde{H}_{r-2}(\hat{0}, x)=$ degree $n$ term in $(-1)^{r} h_{n-r}\left[\sum_{m \geq 1}(-1)^{m-1} \operatorname{ch} \tilde{H}_{m-3}\left(\bar{\Pi}_{m}\right)\right]$. 
Exercise 5.1.13 $([\mathbf{1 7 5}, \mathbf{1 7 6}])$. Use Theorems 5.1.5 and 5.1.9 to compute the representation of $\mathbf{X}_{i} \mathfrak{S}_{m_{i}}\left[\mathfrak{S}_{i}\right]$ on $H_{i}(\hat{0}, x)$, where $m_{i}$ is equal to the number of blocks of $x$ of size $i$ and $(\hat{0}, x)$ is an interval in the

(a) $d$-divisible partition lattice $\Pi_{n d}^{0 \bmod d}$,

(b) $1 \bmod d$ partition poset $\Pi_{n d+1}^{1 \bmod d}$.

Computations such as those of Example 5.1.12 and Exercise 5.1.13 are used in applications of equivariant versions of the Orlik-Solomon formula and the GoreskyMacPherson formula. This is discussed further in Section 5.4.

The examples above don't adequately demonstrate the power of the product theorems because in these examples homology occurs in only one dimension. A demonstration of the full power can be found in [176], $[\mathbf{1 8 2}]$ and $[\mathbf{1 9 9}]$, where representations on the homology of intervals of the $k$-equal partition lattice $\Pi_{n, k}$, the at least $k$ partition lattice $\Pi_{\bar{n}}^{\geq k}$, the general $j \bmod d$ partition poset $\Pi_{n d+j}^{j \bmod d}$, and the other restricted block size partition posets of Section 4.4, are computed.

\subsection{Quillen fiber lemma}

In a seminal 1978 paper, Quillen introduced several poset fiber theorems. In this section we shall present the most basic of these, known as the "the Quillen fiber lemma", which has proved to be one of the most useful tools in poset topology.

Given two posets $P$ and $Q$, a map $f: P \rightarrow Q$ is called a poset map if it is order preserving, i.e., $x \leq_{P} y$ implies $f(x) \leq_{Q} f(y)$. Given two $G$-posets $P$ and $Q$, a poset map $f: P \rightarrow Q$ is called a G-poset map if $g f(x)=f(g x)$ for all $x \in P$. Given two simplicial complexes $\Delta$ and $\Gamma$, a simplicial map is a map $f: \Delta \rightarrow \Gamma$ that takes 0 -faces of $\Delta$ to 0 -faces of $\Gamma$ and preserves inclusions, i.e., $f$ is a poset map from $P(\Delta)$ to $P(\Gamma)$. If $\Delta$ and $\Gamma$ are $G$-simplicial complexes and $f$ commutes with the $G$-action then $f$ is said to be a $G$-simplicial map. A $G$-continuous map $f: X \rightarrow Y$ from $G$-space $X$ to $G$-space $Y$ is a continuous map that commutes with the $G$-action. Clearly, a $G$-poset map $f: P \rightarrow Q$ induces a $G$-simplicial map $f: \Delta(P) \rightarrow \Delta(Q)$, which in turn induces a $G$-continuous map $f:\|\Delta(P)\| \rightarrow\|\Delta(Q)\|$.

For any element $x$ of a poset $P$, let

$$
P_{>x}:=\{y \in P: y>x\} \text { and } P_{\geq x}:=\{y \in P: y \geq x\} .
$$

The subsets $P_{<x}$ and $P_{\leq x}$ are defined similarly.

The basic version of the Quillen fiber lemma pertains to homotopy type. Quillen also gave an integral homology version and a equivariant homology version. There is also an equivariant homotopy version due to Thévenaz and Webb [187]. We present only the homotopy version and the equivariant homology version. Recall that $\simeq$ denotes homotopy equivalence.

Theorem 5.2.1 (Quillen fiber lemma [135]). Let $f: P \rightarrow Q$ be a poset map. If the fiber $f^{-1}\left(Q_{\leq y}\right)$ is contractible for all $y \in Q$ then

$$
P \simeq Q .
$$

Theorem 5.2.2 (Equivariant homology version [135]). Let $f: P \rightarrow Q$ be a $G$-poset map. If the fiber $f^{-1}\left(Q_{\leq y}\right)$ is acyclic (over $\mathbb{C}$ ) for all $y \in Q$ then

$$
\tilde{H}_{r}(P) \cong_{G} \tilde{H}_{r}(Q)
$$

for all $r$. 
Let's look at Quillen's original example [135]. For a finite group $G$, let $\mathcal{S}_{p}(G)$ be the poset of nontrivial $p$-subgroups of $G$ ordered by inclusion and let $\mathcal{A}_{p}(G)$ be the induced subposet of nontrivial elementary abelian $p$-subgroups of $G$. The poset $\mathcal{S}_{p}(G)$ and its order complex were studied by Brown in his work on group cohomology. Quillen proposed the smaller poset $\mathcal{A}_{p}(G)$ as a way of studying the homotopy invariants of the Brown complex. He used the Quillen fiber lemma to establish homotopy equivalence between the Brown complex and the Quillen complex. Both posets are $G$-posets, where $G$ acts by conjugation.

Theorem 5.2.3 (Quillen $[\mathbf{1 3 5}]$ ). For any finite group $G$ and prime $p$,

$$
\mathcal{A}_{p}(G) \simeq \mathcal{S}_{p}(G),
$$

and

$$
\tilde{H}_{j}\left(\mathcal{A}_{p}(G)\right) \cong_{G} \tilde{H}_{j}\left(\mathcal{S}_{p}(G)\right) \quad \forall j .
$$

Proof. The inclusion map

$$
i: \mathcal{A}_{p}(G) \rightarrow \mathcal{S}_{p}(G)
$$

is a $G$-poset map. Let us show that the fiber $i^{-1}\left(S_{p}(G)_{\leq H}\right)$ is contractible for all $H \in \mathcal{S}_{p}(G)$ so that we can apply the Quillen fiber lemma. Note that

$$
i^{-1}\left(S_{p}(G)_{\leq H}\right)=\mathcal{A}_{p}(H) .
$$

Since $H$ is a nontrivial $p$-group, it has a nontrivial center. Let $B$ be the subgroup of the center consisting of all elements of order 1 or $p$. Consider the poset map $f: \mathcal{A}_{p}(H) \rightarrow\left\{B A: A \in \mathcal{A}_{p}(H)\right\}$ defined by $f(A)=B A$. The fibers of this map are contractible since they all have maximum elements. So by the Quillen fiber lemma

$$
\mathcal{A}_{p}(H) \simeq\left\{B A: A \in \mathcal{A}_{p}(H)\right\} .
$$

Since $\left\{B A: A \in \mathcal{A}_{p}(H)\right\}$ has minimum element $B$, it is contractible. So $\mathcal{A}_{p}(H)$ and thus $i^{-1}\left(S_{p}(G)_{\leq H}\right)$ is contractible. Hence by the Quillen fiber lemma, (5.2.1) holds and by the equivariant homology version, (5.2.2) holds.

For certain finite groups $G$, the homology of the Quillen complex $\Delta\left(\mathcal{A}_{p}(G)\right)$ is well-understood; namely for groups of Lie type. Quillen [135] shows that for groups $G$ of Lie type in characteristic $p$, the Quillen complex $\Delta\left(\mathcal{A}_{p}(G)\right)$ is homotopic to a simplicial complex known as the building for $G$, which has the homotopy type of a wedge of spheres of a single dimension. Webb [207] shows that the unique nonvanishing $G$-equivariant homology of $\mathcal{A}_{p}(G)$ is the same as that of the building, which is known as the Steinberg representation. For general finite groups, the Quillen complex is not nearly as well-behaved, nor well-understood. In fact, it was only recently shown by Shareshian $[\mathbf{1 5 4}]$ that for certain primes the integral homology of the Quillen complex of the symmetric group has torsion.

The following long-standing conjecture of Quillen imparts a sense of the significance of poset topology in group theory.

Conjecture 5.2.4 (Quillen Conjecture). For any finite group $G$ and prime $p$, the poset $\mathcal{A}_{p}(G)$ is contractible if and only if $G$ has a nontrivial normal p-subgroup.

The necessity of contractibility was proved by Quillen; the sufficiency is still open. However significant progress has been made by Aschbacher and Smith [4].

In order to gain understanding of the Quillen complex for the symmetric group, Bouc [47] considered the induced subposet $T_{n}$ of $\mathcal{S}_{2}\left(\mathfrak{S}_{n}\right)$ consisting of nontrivial 
2-subgroups of $\mathfrak{S}_{n}$ that contain a transposition, and the induced subposet $T_{n}^{\prime}$ of $\mathcal{A}_{2}\left(\mathfrak{S}_{n}\right)$ consisting of nontrivial elementary abelian 2-subgroups generated by transpositions. It is easy to see that $T_{n}^{\prime}$ is $\mathfrak{S}_{n}$-homeomorphic to the matching complex $M_{n}$ discussed in Section 2.4.3. Bouc used the Quillen fiber lemma to prove

$$
T_{n} \simeq T_{n}^{\prime} \simeq M_{n}
$$

and for all $i$,

$$
\tilde{H}_{i}\left(T_{n}\right) \cong_{\mathfrak{S}_{n}} \tilde{H}_{i}\left(T_{n}^{\prime}\right) \cong_{\mathfrak{S}_{n}} \tilde{H}_{i}\left(M_{n}\right) .
$$

In addition to computing the representation of the symmetric group on the homology of the matching complex, Bouc discovered torsion in the integral homology of the matching complex. See $[\mathbf{1 5 5}],[\mathbf{2 0 0}]$ and $[\mathbf{1 0 3}]$ for further results on torsion in the matching complex.

Exercise 5.2.5. Prove (5.2.3) and (5.2.4).

The usefulness of the matching complex in understanding the topology of the Quillen and Brown complexes was recently demonstrated by Ksontini $[\mathbf{1 1 4}, \mathbf{1 1 5}]$. He used simple connectivity of $M_{n}$ for $n \geq 8$, which was proved by Bouc, to establish simple connectivity of $\mathcal{S}_{2}\left(\mathfrak{S}_{n}\right)$ for $n \geq 8$. It was also shown by Ksontini $[\mathbf{1 1 4}, \mathbf{1 1 5}$, 116], Shareshian [154], and Shareshian and Wachs [156] that a hypergraph version of the matching complex is useful in studying the topology of $\Delta\left(\mathcal{S}_{p}\left(\mathfrak{S}_{n}\right)\right)$ when $p \geq 3$.

Exercise 5.2.6. Let $\bar{x}$ be a closure operator on $P$, i.e. $x \leq \bar{x}$ and $\overline{\bar{x}}=\bar{x}$ for all $x \in P$. Define $\operatorname{cl}(P):=\{x \in P: \bar{x}=x\}$.

(a) Use the Quillen fiber lemma to prove:

$$
P \simeq \operatorname{cl}(P) .
$$

(b) Use (a) to show that

$$
\overline{\mathcal{W}}(n, k) \simeq \overline{\mathcal{N}}(n, k) .
$$

(We already know this and (c) from Exercise 4.3.2.)

(c) Derive an equivariant homology version of the homotopy result in (a) and use it to prove

$$
\tilde{H}_{i}(\overline{\mathcal{W}}(n, k)) \cong_{\mathfrak{S}_{n}} \tilde{H}_{i}(\overline{\mathcal{N}}(n, k)) \quad \forall i .
$$

Exercise 5.2.7 (Homology version of Rota's crosscut theorem). Let $L$ be a lattice and let $M$ be the subposet of $\bar{L}$ consisting of non-ô meets of coatoms. Prove:

(a) $\bar{L} \simeq M$.

(b) If $G$ is a group acting on $L$ then for all $i, \tilde{H}_{i}(\bar{L}) \cong_{G} \tilde{H}_{i}(M)$.

(c) Let $\Gamma(L)$ be the simplicial complex whose vertex set is the set of coatoms of $L$ and whose faces are sets of coatoms whose meet is not $\hat{0}$ (this is known as the cross-cut complex of $L)$. Show $\bar{L} \simeq \Gamma(L)$ and if $G$ is a group acting on $L$ then for all $i, \tilde{H}_{i}(\bar{L}) \cong_{G} \tilde{H}_{i}(\Gamma(L))$.

The next two examples are connected with the combinatorics of knot spaces and arose in the work of Vassiliev $[\mathbf{1 9 1}, \mathbf{1 9 2}, \mathbf{1 9 3}]$. 
Example 5.2.8. Let $\mathrm{NCG}_{n}$ be the poset of disconnected graphs on node set $[n]$ ordered by inclusion of edge sets. Let

$$
f: \overline{\mathrm{NCG}}_{n} \rightarrow \bar{\Pi}_{n}
$$

be the poset map such that $f(G)$ is the partition of $[n]$ whose blocks are the node sets of the connected components of $G$. The fibers of $f$ are given by

$$
f^{-1}((\hat{0}, \pi])=\left(\hat{0}, G_{\pi}\right],
$$

where $G_{\pi}$ is the graph whose connected components are cliques on the blocks of $\pi$. Since the fibers have maximal elements they are contractible. Hence by the Quillen fiber lemma

$$
\overline{\mathrm{NCG}}_{n} \simeq \bar{\Pi}_{n},
$$

which implies that $\overline{\mathrm{NCG}}_{n}$ has the homotopy type of a wedge of $(n-1)$ ! spheres of dimension $n-3$. By the equivariant homology version of the Quillen fiber lemma the only nonvanishing homology of $\overline{\mathrm{NCG}}_{n}$ is given by

$$
\tilde{H}_{n-3}\left(\overline{\mathrm{NCG}}_{n}\right) \cong_{\mathfrak{S}_{n}} \tilde{H}_{n-3}\left(\Pi_{n}\right) .
$$

Exercise 5.2.9. Let $\mathrm{CG}_{n}$ be the poset of connected graphs on node set $[n]$ ordered by inclusion of edge sets. From Alexander duality and (5.2.5), we have that he only nonvanishing homology of $\overline{\mathrm{CG}}_{n}$ is given by

$$
\tilde{H}_{\left(\begin{array}{c}
n-1 \\
2
\end{array}\right)-1}\left(\overline{\mathrm{CG}}_{n}\right) \cong_{\mathfrak{S}_{n}} \operatorname{sgn}^{\otimes n} \otimes \tilde{H}_{n-3}\left(\Pi_{n}\right) .
$$

Show that for $n \geq 3$, the poset $\overline{\mathrm{CG}}_{n}$ has the homotopy type of a wedge of $(n-1)$ ! spheres of dimension $\left(\begin{array}{c}n-1 \\ 2\end{array}\right)-1$.

Example 5.2.10. A graph is said to be $k$-connected if removal of any set of at most $k-1$ vertices leaves the graph connected. Note that, unless $k=1$, this is a different graph property from that of being $k$-edge-connected, which was discussed in Section 4.5.2. Every triangle tree is 2-edge-connected, but not 2-connected if the number of nodes is greater than 3. Let $\mathrm{NCG}_{n}^{k}$ be the poset of graphs on node set $[n]$ that are not $k$-connected, ordered by inclusion of edge sets. The following result solves a problem of Vassiliev [193] which arose from his study of knots.

Theorem 5.2.11 (Babson, Björner, Linusson, Shareshian, Welker [8], Turchin [190]).

(a) $\overline{N C G}_{n}^{2}$ has the homotopy type of a wedge of $(n-2)$ ! spheres of dimension $2 n-5$.

(b) $\tilde{H}_{n-3}\left(\overline{N C G}_{n}^{2}\right) \downarrow \mathfrak{S}_{\mathfrak{S} n-1}^{\mathfrak{S}_{n}} \cong \mathfrak{S}_{n-1} \quad \tilde{H}_{n-4}\left(\Pi_{n-1}\right) \otimes \operatorname{sgn}_{n-1}$

(c) $\tilde{H}_{n-3}\left(\overline{N C G}_{n}^{2}\right) \cong_{\mathfrak{S}_{n}}\left(\tilde{H}_{n-4}\left(\Pi_{n-1}\right) \uparrow \mathfrak{S}_{\mathfrak{S}_{n-1}}-\tilde{H}_{n-3}\left(\Pi_{n}\right)\right) \otimes \operatorname{sgn}_{n}$

Note that the representation on the right side of (c) is the Whitehouse module discussed in Section 4.5.2. We describe the proof method of Babson, Björner, Linusson, Shareshian, and Welker. Define the poset map

$$
f: \overline{\mathrm{NCG}}_{n}^{2} \rightarrow \overline{B_{n-1} \times \Pi_{n-1}}
$$

by letting

$$
f(G)=(S, \pi)
$$




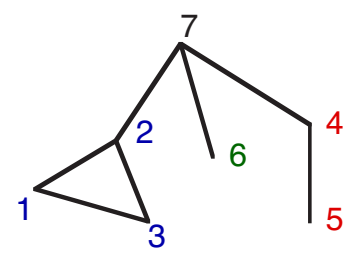

Figure 5.2.1. Not 2-connected graph

where $S$ is the set of nodes joined to node $n$ by an edge, and $\pi$ is the partition whose blocks correspond to the connected components of $G \backslash\{n\}$. For example, if $G$ is the not 2-connected graph in Figure 5.2.1 then

$$
f(G)=(\{2,4,6\}, 123 / 45 / 6) .
$$

Some of the fibers have maximum elements; so one sees immediately that they are contractible. For example,

$$
f^{-1}((\hat{0},(\{2,4,6\}, 123 / 45 / 6)])=(\hat{0}, G],
$$

where $G$ is the graph in Figure 5.2.1. But it is not so easy to see that the fibers without maximum elements are contractible. For example, the fiber $f^{-1}((\hat{0},(\{1,2\}, 123456)])$ has no maximum. To show that such fibers are contractible, Babson, Björner, Linusson, Shareshian, and Welker used discrete Morse theory, which had just been introduced by Forman [72]. In fact, this was the first of many applications of discrete Morse theory in topological combinatorics.

Now by the Quillen fiber lemma, we have

$$
\overline{\mathrm{NCG}}_{n}^{2} \simeq \overline{B_{n-1} \times \Pi_{n-1}} \text {. }
$$

Since the product of shellable bounded posets is shellable (Theorem 3.1.10), and the proper part of a shellable poset is shellable (Corollary 3.1.9), $\overline{B_{n-1} \times \Pi_{n-1}}$ has the homotopy type of a wedge of $(2 n-5)$-spheres. The number of spheres is the absolute value of the Möbius invariant, which by Proposition 1.2.1 and (3.2.1) is $(n-2)$ !. Hence Part (a) of the theorem holds.

For Part (b), we use the equivariant homology version of the Quillen fiber lemma. Clearly the map $f$ commutes with the permutations that fix $n$. Hence,

$$
\tilde{H}_{2 n-5}\left({\overline{\mathrm{NCG}_{n}}}_{n}^{2}\right) \downarrow_{\mathfrak{S}_{n-1}}^{\mathfrak{S}_{n-1}} \cong \mathfrak{S}_{n-1} \tilde{H}_{2 n-5}\left(\overline{B_{n-1} \times \Pi_{n-1}}\right) .
$$

Theorem 5.1.5 completes the proof of Part (b).

The proof of Part (c) uses Part (b) and a computation of the fixed point Möbius invariant (Section 4.6).

Exercise 5.2.12. Let $\mathrm{CG}_{n}^{k}$ be the poset of $k$-connected graphs on node set $[n]$ ordered by inclusion of edge sets.

(a) Use Theorem 5.2.11 to determine the homotopy type of $\overline{\mathrm{CG}}_{n}^{2}$.

(b) ([8]) Show that $\overline{\mathrm{CG}}_{n}^{n-2}$ is dual to the face poset of the matching complex $M_{n}$. Conclude that neither $\overline{\mathrm{CG}}_{n}^{n-2}$ nor $\overline{\mathrm{NCG}}_{n}^{n-2}$ has the homotopy type of a wedge of spheres and that their integral homology has torsion. 
Shareshian [152] uses discrete Morse theory to determine the homotopy type of $\overline{\mathrm{CG}}_{n}^{2}$ directly without resorting to Theorem 5.2.11, and to obtain bases for homology of $\overline{\mathrm{CG}}_{n}^{2}$, thereby solving another problem of Vassiliev [193]. The only value of $k$ other than $k=1,2, n-2$, for which anything significant is known about the topology of the not $k$-connected graph complex is $k=3$. Discrete Morse theory is used to obtain the following result.

Theorem 5.2.13 (Jonsson [102]). $\overline{N C G}_{n}^{3}$ has the homotopy type of a wedge of $(n-3)(n-2) ! / 2$ spheres of dimension $2 n-4$.

Problem 5.2.14. What can be said about the representation of $\mathfrak{S}_{n}$ on the homology of $\overline{\mathrm{NCG}}_{n}^{3}$.

Problem 5.2.15. ([8]) What can be said about the topology of $\overline{\mathrm{NCG}}_{n}^{k}$ for $3<$ $k<n-2$. When does $\overline{\mathrm{NCG}}_{n}^{k}$ fail to be a wedge of spheres? When does its integral homology have torsion? Recall that the integral homology of $\overline{\mathrm{NCG}}_{n}^{n-2}$ has torsion (Exercise 5.2.12 (b)).

\subsection{General poset fiber theorems}

In this section we present homotopy and homology versions of a fiber theorem of Björner, Wachs and Welker, which generalizes the Quillen fiber lemma and several other fiber theorems. Recall the definitions of $k$-connectivity and $k$-acyclicity, which appear after Theorem 3.1.7.

Theorem 5.3.1 (Björner, Wachs, Welker [43]). Let $f: P \rightarrow Q$ be a poset map such that for all $q \in Q$ the fiber $\Delta\left(f^{-1}\left(Q_{\leq q}\right)\right)$ is $l\left(f^{-1}\left(Q_{<q}\right)\right)$-connected. Then

$$
\Delta(P) \simeq \Delta(Q) \vee\left\{\Delta\left(f^{-1}\left(Q_{\leq q}\right)\right) * \Delta\left(Q_{>q}\right): q \in Q\right\},
$$

where * denotes join defined in (1.1.1) and $\vee$ denotes the wedge (of $\Delta(Q)$ and all $\left.\Delta\left(f^{-1}\left(Q_{\leq q}\right)\right) * \Delta\left(Q_{>q}\right)\right)$ formed by identifying the vertex $q$ in $\Delta(Q)$ with any element of $f^{-1}\left(Q_{\leq q}\right)$, for each $q \in Q$.

For clarity, let us remark that if $\Delta(Q)$ is connected then the space described on the right-hand side of (5.3.1), which has $|Q|$ wedge-points, is homotopy equivalent to a one-point wedge, where arbitrarily chosen points of $f^{-1}\left(Q_{\leq q}\right)$, one for each $q \in Q$, are identified with some (arbitrarily chosen) point of $Q$. Thus (5.3.1) becomes

$$
\Delta(P) \simeq \Delta(Q) \vee \bigvee_{q \in Q} \Delta\left(f^{-1}\left(Q_{\leq q}\right)\right) * \Delta\left(Q_{>q}\right)
$$

We will refer to a poset map $f: P \rightarrow Q$ such that for all $q \in Q$ the fiber $\Delta\left(f^{-1}\left(Q_{\leq q}\right)\right)$ is $l\left(f^{-1}\left(Q_{<q}\right)\right)$-connected as being well-connected. Note that the connectivity condition implies that each fiber $f^{-1}\left(Q_{\leq q}\right)$ is nonempty.

Example 5.3.2. Let $f: P \rightarrow Q$ be the poset map depicted in Figure 5.3.1. For the two maximal elements of $Q$ the fiber $\Delta\left(f^{-1}\left(Q_{\leq q}\right)\right)$ is a 1 -sphere. For the bottom element of $Q$ the fiber $\Delta\left(f^{-1}\left(Q_{\leq q}\right)\right)$ is a 0 -sphere, and $\Delta\left(Q_{>q}\right)$ is a 0 -sphere too. So in either case $\Delta\left(f^{-1}\left(Q_{\leq q}\right)\right) * \Delta\left(Q_{>q}\right)$ is homeomorphic to a 1 -sphere. Hence the simplicial complex on the right side of (5.3.1) has a 1-sphere attached to each element of $Q$. Thus Theorem 5.3.1 determines $\Delta(P)$ to have the homotopy type of a wedge of three 1-spheres. One can see this directly by observing that $\Delta(P)$ is homeomorphic to two 1-spheres intersecting in two points. 


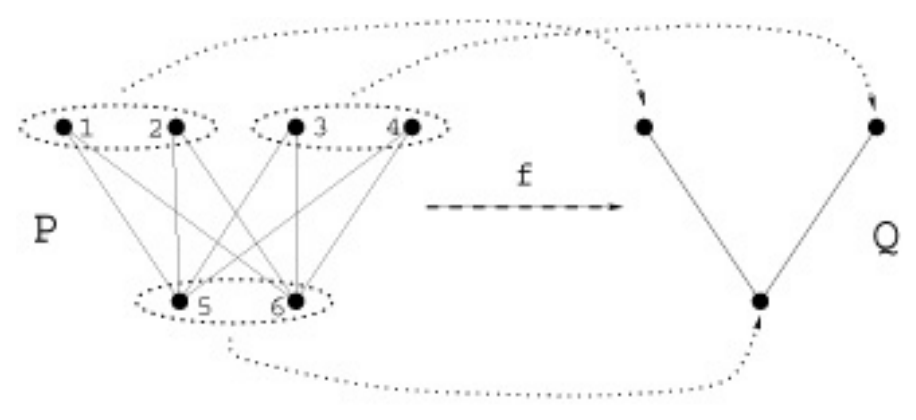

Figure 5.3.1. A well-connected poset map

In [43], a version of the general fiber theorem for homology over the integers or over any field is also given, as are equivariant homotopy and homology versions. We state only the equivariant homology version here.

Theorem 5.3.3 (Björner, Wachs, Welker [43]). Let $f: P \rightarrow Q$ be a $G$-poset map. If $f^{-1}\left(Q_{\leq q}\right)$ is $l\left(f^{-1}\left(Q_{<y}\right)\right)$-acyclic for all $q \in Q$ then

$$
\tilde{H}_{r}(P) \cong_{G} \tilde{H}_{r}(Q) \oplus \bigoplus_{q \in Q / G} \bigoplus_{i=-1}^{r}\left(\tilde{H}_{i}\left(f^{-1}\left(Q_{\leq q}\right)\right) \otimes \tilde{H}_{r-i-1}\left(Q_{>q}\right)\right) \uparrow_{G_{q}}^{G} .
$$

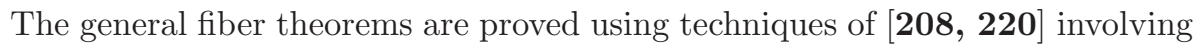
diagrams of spaces and spectral sequences, which were developed to study subspace arrangements. In Section 5.4, we discuss the connection between the general fiber theorems and subspace arrangements.

Example 5.3.4. Theorem 5.3 .3 can be applied to the well-connected poset map $f: P \rightarrow Q$ given in Example 5.3.2. Let $G$ be the cyclic group $\mathfrak{S}_{2}$ whose nonidentity element acts by $(1,2)(3,4)$ on $P$ and trivially on $Q$. The map $f$ is clearly a $G$-poset map. For each $q \in Q$, we have $G_{q}=\mathfrak{S}_{2}$. If $q$ is the bottom element of $Q$ then the fiber $f^{-1}\left(Q_{\leq q}\right)$ is $\mathfrak{S}_{2}$-homeomorphic to a 0 -sphere on which $\mathfrak{S}_{2}$ acts trivially. The same is true for $Q_{>q}$. It follows that the representation of $\mathfrak{S}_{2}$ on $\tilde{H}_{0}\left(f^{-1}\left(Q_{\leq q}\right)\right) \otimes \tilde{H}_{0}\left(Q_{>q}\right)$ is the trivial representation $S^{(2)}$. If $q$ is one of the maximal elements of $Q$ then the fiber $f^{-1}\left(Q_{\leq q}\right)$ is $\mathfrak{S}_{2}$-homeomorphic to a circle with $(1,2)(3,4)$ acting by reflecting the circle about the line spanned by a pair of antipodal points. Hence the representation of $\mathfrak{S}_{2}$ on $\tilde{H}_{1}\left(f^{-1}\left(Q_{\leq q}\right)\right)$ is the sign representation $S^{\left(1^{2}\right)}$. Since $Q_{>q}$ is the empty simplicial complex, the representation of $\mathfrak{S}_{2}$ on $\tilde{H}_{-1}\left(Q_{>q}\right)$ is the trivial representation. It follows that the representation of $\mathfrak{S}_{2}$ on $\tilde{H}_{1}\left(f^{-1}\left(Q_{<q}\right)\right) \otimes \tilde{H}_{-1}\left(Q_{>q}\right)$ is $S^{\left(1^{2}\right)}$. We conclude from (5.3.3) that the $\mathfrak{S}_{2}$-module $\tilde{H}_{1}(P)$ decomposes into $S^{(2)} \oplus S^{\left(1^{2}\right)} \oplus S^{\left(1^{2}\right)}$ and that $\tilde{H}_{i}(P)=0$ for $i \neq 1$.

Example 5.3.5. Consider the action of the hyperoctahedral group $\mathfrak{S}_{n}\left[\mathbb{Z}_{2}\right]$ on the type B partition lattice $\Pi_{n}^{B}$. Define the bar-erasing map

$$
f: \bar{\Pi}_{n}^{B} \rightarrow \bar{\Pi}_{\{0,1, \ldots, n\}}
$$




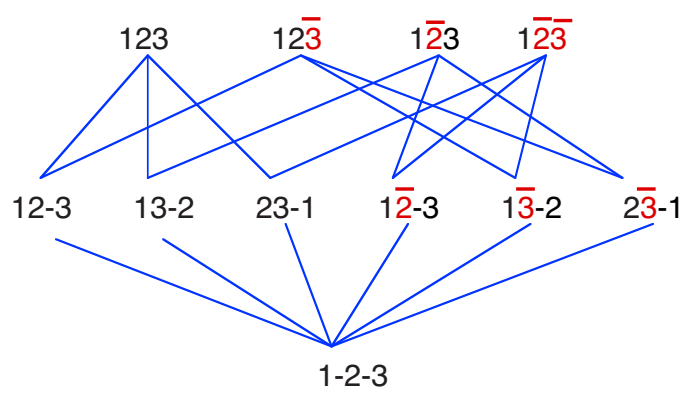

Figure 5.3.2. $P_{3}$

by letting $f(\pi)$ be the partition obtained by erasing the bars from the barred partition $\pi$. For example

$$
f(047 / 1 \overline{5} 6 \overline{9} / 2 \overline{3})=047 / 1569 / 23
$$

It is clear that $f$ is a $\mathfrak{S}_{n}\left[\mathbb{Z}_{2}\right]$-poset map. We establish well-connectedness in the following exercise.

Exercise 5.3.6 (Wachs $[202]$ ). Let $P_{n}$ be the subposet of $\Pi_{n}^{B}$ consisting of barred partitions whose zero-block is a singleton, where the zero-block is the block that contains 0. The poset $P_{3}$ with the zero-block suppressed is given in Figure 5.3.2. Show the following.

(a) The fibers of the bar-erasing map have the form

$$
\Pi_{b_{0}}^{B} \times P_{b_{1}} \times \cdots \times P_{b_{k}}-\{\hat{0}\},
$$

where $b_{0}+b_{1}+\cdots+b_{k}=n+1$.

(b) $P_{n}$ is a geometric semilattice

(c) The bar-erasing map (5.3.4) is well-connected.

(d) $\mid \mu\left(P_{n} \cup\{\hat{1}\} \mid=(2(n-1)-1) !\right.$ !.

It follows from Exercise 5.3.6 that the general fiber theorems can be used to compute the homotopy type and $\mathfrak{S}_{n}\left[\mathbb{Z}_{2}\right]$-equivariant homology of $\Pi_{n}^{B}$. Since $\overline{\Pi_{n}^{B}}$ has the homotopy type of a wedge of $(2 n-1)$ !! spheres of dimension $n-2$ (see Exercise 3.2.11), the homotopy equivalence (5.3.2) reduces to a combinatorial identity, which turns out to be easy to prove directly and therefore not very interesting. The equivariant homology version of the general fiber theorem does yield an interesting identity. Theorems 5.1.5 and 5.1.9 can be applied to the fibers given in Part (a) of Exercise 5.3.6. This and the homology version of the general fiber theorem are used in $[\mathbf{2 0 2}]$ to prove

$$
\tilde{H}_{n-2}\left(\overline{\Pi_{n}^{B}}\right) \cong_{\mathfrak{S}_{n}\left[\mathbb{Z}_{2}\right]} \bigoplus_{\left(\gamma_{1}, \ldots, \gamma_{k}\right): \sum_{i} \gamma_{i}=n} \tilde{H}_{\gamma_{1}-2}\left(\overline{P_{\gamma_{1}}}\right) \bullet \cdots \bullet \tilde{H}_{\gamma_{k}-2}\left(\overline{P_{\gamma_{k}}}\right),
$$

where $\bullet$ denotes induction product:

$$
X \bullet Y:=(X \otimes Y) \uparrow \underset{\mathfrak{S}_{n}\left[\mathbb{Z}_{2}\right] \times \mathfrak{S}_{m}\left[\mathbb{Z}_{2}\right]}{\mathfrak{S}_{n+m}\left[\mathbb{Z}_{2}\right]} .
$$

This example is considered in the more general setting of rank-selected Dowling lattices in [202]. 
Nonpure versions of Cohen-Macaulay fiber theorems of Baclawski [12] and of Quillen [135] follow from the general fiber theorems. We state only the nonpure version of Baclawski's result.

Theorem 5.3.7 (Björner, Wachs, Welker $[43]$ ). Let $P$ and $Q$ be semipure posets and let $f: P \rightarrow Q$ be a surjective rank-preserving poset map. Assume that for all $q \in Q$, the fiber $\Delta\left(f^{-1}\left(Q_{\leq q}\right)\right)$ is Cohen-Macaulay over $\mathbf{k}$. If $Q$ is sequentially Cohen-Macaulay over $\mathbf{k}$, then so is $P$.

\subsection{Fiber theorems and subspace arrangements}

Shortly after the Goresky-MacPherson formula (Theorem 1.3.8) appeared, Ziegler and Živaljević obtained a homotopy version, and after that Sundaram and Welker obtained an equivariant homology version. In this section we show that the ZieglerŽivaljević and Sundaram-Welker formulas for linear subspace arrangements can be easily derived from the general fiber theorems of Section 5.3. This connection should come as no surprise, since the method of proof of the general fiber theorems is based on the proofs of Ziegler-Živaljević and Sundaram-Welker; namely diagrams of spaces and spectral sequences.

Let $\mathcal{A}$ be a linear subspace arrangement i.e., a finite collection of linear subspaces in Euclidean space $\mathbb{R}^{d}$. The singularity link $V_{\mathcal{A}}^{o}$ is defined as

$$
V_{\mathcal{A}}^{o}=\mathbb{S}^{(d-1)} \cap \bigcup_{X \in \mathcal{A}} X
$$

where $\mathbb{S}^{(d-1)}$ is the unit $(d-1)$-sphere in $\mathbb{R}^{d}$. Recall that $L(\mathcal{A})$ denotes the intersection lattice of $\mathcal{A}$ (ordered by reverse inclusion). Let $\bar{L}(\mathcal{A})$ denote $L(\mathcal{A}) \backslash\{\hat{0}, \hat{1}\}$ if $\mathcal{A}$ is essential (i.e., $\cap \mathcal{A}=\{0\}$ ), and $L(\mathcal{A}) \backslash\{\hat{0}\}$ otherwise.

Theorem 5.4.1 (Ziegler \& Živaljević $[\mathbf{2 2 0}]$ ). Let $\mathcal{A}$ be a linear subspace arrangement. Then

$$
V_{\mathcal{A}}^{o} \simeq \Delta(\bar{L}(\mathcal{A})) \vee\left\{\operatorname{susp}^{\operatorname{dim} x}(\Delta(\hat{0}, x)): x \in \bar{L}(\mathcal{A})\right\},
$$

where the wedge is formed by identifying each vertex $x$ in $\Delta(\bar{L}(\mathcal{A}))$ with any point in $\operatorname{susp}^{\operatorname{dim} x}(\Delta(\hat{0}, x))$. Consequently, for each $i$,

$$
\tilde{H}_{i}\left(V_{\mathcal{A}}^{o} ; \mathbb{Z}\right) \cong \bigoplus_{x \in L(\mathcal{A}) \backslash\{\hat{0}\}} \tilde{H}_{i-\operatorname{dim} x}((\hat{0}, x) ; \mathbb{Z}) .
$$

Proof. (given in $[\mathbf{4 3}]$ ). Suppose $\mathcal{A}=\left\{X_{1}, \ldots, X_{n}\right\}$. Let $\mathcal{H}$ be an essential hyperplane arrangement in $\mathbb{R}^{d}$ such that each $X_{i}$ is the intersection of a collection of hyperplanes in $\mathcal{H}$. The hyperplane arrangement $\mathcal{H}$ partitions $\mathbb{S}^{(d-1)}$ into open cells of each dimension from 0 to $d-1$. Let $\mathcal{F}(\mathcal{H})$ be the poset of closures of the nonempty cells ordered by inclusion. Clearly $\Delta(\mathcal{F}(\mathcal{H}))$ is a triangulation of $\mathbb{S}^{(d-1)}$. For $X \in \bar{L}(\mathcal{A})$, let $\mathcal{F}_{\mathcal{H}}(X)$ be the order ideal of $\mathcal{F}(\mathcal{H})$ consisting of closed cells contained in $X$, and let $\mathcal{F}_{\mathcal{H}}(\mathcal{A})$ be the order ideal of $\mathcal{F}(\mathcal{H})$ consisting of closed cells contained in $V_{\mathcal{A}}^{o}$. Note that $\Delta\left(\mathcal{F}_{\mathcal{H}}(X)\right)$ is a triangulation of the $(\operatorname{dim} X-1)$-sphere $X \cap \mathbb{S}^{(d-1)}$ and $\Delta\left(\mathcal{F}_{\mathcal{H}}(\mathcal{A})\right)$ is a triangulation of $V_{\mathcal{A}}^{o}$.

Now let

$$
f: \mathcal{F}_{\mathcal{H}}(\mathcal{A}) \rightarrow \bar{L}(\mathcal{A})^{*}
$$


be defined by

$$
f(\tau)=\bigcap_{i: X_{i} \supseteq \tau} X_{i}
$$

Clearly $f$ is order preserving. We claim that $f$ is well-connected. Observe that for all $X \in \bar{L}(\mathcal{A})$,

$$
f^{-1}\left(\left(\bar{L}(\mathcal{A})^{*}\right)_{\leq X}\right)=\mathcal{F}_{\mathcal{H}}(X)
$$

So $f^{-1}\left(\left(\bar{L}(\mathcal{A})^{*}\right)_{<X}\right)$ is a $(\operatorname{dim} X-1)$-sphere, which is $(\operatorname{dim} X-2)$-connected. Since $f^{-1}\left(\left(\bar{L}(\mathcal{A})^{*}\right)_{<X}\right)$ has length at most $\operatorname{dim} X-2, f$ is indeed well-connected, and thus (5.4.1) follows from Theorem 5.3.1.

The reason we refer to Theorem 5.4.1 as a homotopy version of the GoreskyMacPherson formula is that the Goresky-MacPherson formula (for linear subspace arrangements) follows from (5.4.2) by Alexander duality. Indeed, one can simply apply Theorem 5.1.1 to the subposet $\mathcal{F}_{\mathcal{H}}(\mathcal{A})$ of $\mathcal{F}(\mathcal{H})$. A homotopy version of the Goresky-MacPherson formula for general affine subspace arrangements is also given in $[\mathbf{2 2 0}]$.

Now let $G$ be a finite subgroup of the orthogonal group $O_{d}$ that maps subspaces in $\mathcal{A}$ to subspaces in $\mathcal{A}$. We say that $\mathcal{A}$ is a $G$-arrangement. Clearly $G$ acts as a group of poset maps on $L(\mathcal{A})$ and as a group of homomorphisms on $\tilde{H}_{i}\left(V_{\mathcal{A}}^{o}\right)$ and on $\tilde{H}_{i}\left(M_{\mathcal{A}}\right)$. For each $x \in L(\mathcal{A})$, let $S_{x}$ be the $(\operatorname{dim} x-1)$-sphere $x \cap \mathbb{S}^{(d-1)}$. Each $g$ in the stabilizer $G_{x}$ acts as an orientation preserving or reversing homeomorphism on $S_{x}$. Define

$$
\operatorname{sgn}_{x}(g):= \begin{cases}1 & \text { if } g \text { is orientation preserving } \\ -1 & \text { if } g \text { is orientation reversing. }\end{cases}
$$

By viewing $g$ as an element of $G L(x)$, we have $\operatorname{sgn}_{x}(g)=\operatorname{det}(g)$. Note that $\tilde{H}_{\operatorname{dim} x-1}\left(S_{x}\right)$ is a one dimensional representation of $G_{x}$ whose character is given by $\operatorname{sgn}_{x}$.

Theorem 5.4.2 (Sundaram and Welker $[\mathbf{1 8 4}]$ ). Let $\mathcal{A}$ be a $G$-arrangement of linear subspaces in $\mathbb{R}^{d}$. Then for all $i$,

$$
\tilde{H}_{i}\left(V_{\mathcal{A}}^{o}\right) \cong_{G} \bigoplus_{x \in L(\mathcal{A}) \backslash\{\hat{0}\} / G}\left(\tilde{H}_{i-\operatorname{dim} x}(\hat{0}, x) \otimes \operatorname{sgn}_{x}\right) \uparrow_{G_{x}}^{G} .
$$

Consequently,

$$
\text { (5.4.4) } \tilde{H}^{i}\left(M_{\mathcal{A}}\right) \cong_{G} \bigoplus_{x \in L(\mathcal{A}) \backslash\{\hat{0}\} / G}\left(\tilde{H}_{d-2-i-\operatorname{dim} x}(\hat{0}, x) \otimes \operatorname{sgn}_{x} \otimes \operatorname{sgn}_{\hat{0}}\right) \uparrow_{G_{x}}^{G} .
$$

Exercise 5.4.3. Use Theorems 5.3.3 and 5.1.1 to prove Theorem 5.4.2.

In [184] Theorem 5.4.2 is applied to subspace arrangements whose intersection lattices are the $k$-equal partition lattice discussed in Section 3.2.4 and the $d$-divisible partition lattice discussed in Exercises 4.3.6 and 4.4.8. By computing the multiplicity of the trivial representation in $\tilde{H}^{i}\left(M_{\mathcal{A}}\right)$, Sundaram and Welker obtain the Betti number formula of Arnol'd given in (3.2.3) and an analogous formula for the space of monic polynomials of degree $n$, with at least one root multiplicity not divisible by $d$. For another recent approach to studying these Betti numbers, see [111].

In [199], Theorem 5.4.2 is applied to the subspace arrangement whose intersection lattice is the restricted block size partition lattice $\Pi_{n}^{S} \cup\{\hat{0}\}$ of Theorem 4.4.11. 
Since each interval $(\hat{0}, x)$ of $\Pi_{n}^{S}$ is the product of smaller $\Pi_{m}^{S}$, one uses Theorem 4.4.11, and the product formulas of Section 5.1 to compute the homology of $(\hat{0}, x)$ (see Example 5.1.12 and Exercise 5.1.13). This yields,

Theorem 5.4.4 (Wachs [199]). Suppose $S \subseteq\{2,3, \ldots\}$ is such that $S$ and $\{s-$ $\min S: s \in S\}$ are closed under addition (eg., $S=\{k, k+1, \ldots\}$ or more generally $S=\{k d,(k+1) d, \ldots\})$. For $n \in S$, let $M_{n}^{S}$ be the manifold

$\left\{\mathbf{z}:=\left(z_{1}, z_{2}, \ldots, z_{n}\right) \in \mathbb{C}^{n}:\right.$ for some $i$ the number of occurrences of $z_{i}$ is not in $\left.S\right\}$.

Then

$$
\sum_{\substack{m \in \mathbb{Z} \\ n \in S}} \operatorname{ch} \tilde{H}^{m}\left(M_{n}^{S}\right) u^{n}(-t)^{2 n-m-1}=\sum_{i \geq 1} h_{i} t^{i}\left[\left(\sum_{i \geq 1} h_{i}\right)^{[-1]}\right]\left[\sum_{i \in S} h_{i} u^{i} t^{\phi(i)}\right],
$$

where $\phi(i):=\max \left\{j \in \mathbb{Z}^{+}: i-(j-1) \min S \in S\right\}$. (Our notation reflects the fact that plethysm is associative.)

We leave it as an open problem to use this formula to compute the multiplicity of the trivial representation in $\tilde{H}^{m}\left(M_{n}^{S}\right)$, and thereby obtain a formula (which generalizes the above mentioned Sundaram-Welker formula) for the Betti numbers of the space of monic polynomials of degree $n$ with at least one root multiplicity not in $S$.

\subsection{Inflations of simplicial complexes}

Let $\Delta$ be a simplicial complex on vertex set $[n]$ and let $\mathbf{m}=\left(m_{1}, \ldots, m_{n}\right)$ be an $n$-tuple of positive integers. We form a new simplicial complex $\Delta_{\mathbf{m}}$, called the $\mathbf{m}$-inflation of $\Delta$, as follows. The vertex set of $\Delta_{\mathbf{m}}$ is $\left\{(i, c): i \in[n], c \in\left[m_{i}\right]\right\}$ and the faces of $\Delta_{\mathbf{m}}$ are of the form $\left\{\left(i_{1}, c_{1}\right), \ldots,\left(i_{k}, c_{k}\right)\right\}$ where $\left\{i_{1}, \ldots, i_{k}\right\}$ is a $(k-1)$-face of $\Delta$ and $c_{j} \in\left[m_{i_{j}}\right]$ for all $j=1, \ldots, k$. We can think of $c_{j}$ as a color assigned to vertex $i_{j}$ and of $\left\{\left(i_{1}, c_{1}\right), \ldots,\left(i_{k}, c_{k}\right)\right\}$ as a coloring of the vertices of face $\left\{i_{1}, \ldots, i_{k}\right\}$. A color for vertex $i$ is chosen from $m_{i}$ colors.

Example 5.5.1. Let $P$ and $Q$ be the posets depicted in Figure 5.3.1. Clearly $\Delta(P)$ is the $(2,2,2)$-inflation of $\Delta(Q)$.

Theorem 5.5.2 (Björner, Wachs, and Welker [43]). Let $\Delta$ be a simplicial complex on vertex set $[n]$ and let $\mathbf{m}$ be an $n$-tuple positive integers. If $\Delta$ is connected then

$$
\Delta_{\mathbf{m}} \simeq \bigvee_{F \in \Delta}\left(\operatorname{susp}^{|F|}\left(l k_{\Delta} F\right)\right)^{\vee \nu(F, \mathbf{m})},
$$

where $\nu(F, \mathbf{m})=\prod_{i \in F}\left(m_{i}-1\right)$, susp ${ }^{k}(\Delta)$ denotes the $k$-fold suspension of $\Delta$, and $\Delta^{\vee k}$ denotes the $k$-fold wedge of $\Delta$.

We prove this theorem in the following exercise.

Exercise 5.5.3. Let $f: \Delta_{\mathrm{m}} \rightarrow \Delta$ be the simplicial map that sends each vertex $(i, c)$ of $\Delta_{\mathrm{m}}$ to vertex $i$ of $\Delta$. We call this the deflating map. This can be viewed as a poset map $f: P\left(\Delta_{\mathbf{m}}\right) \rightarrow P(\Delta)$.

(a) Show that the fiber $f^{-1}\left(P(\Delta)_{\leq F}\right)$ is a geometric semilattice.

(b) Show that each $f^{-1}\left(\overline{P(\Delta)}_{\leq F}\right)$ is a wedge of $\nu(F, \mathbf{m})$ spheres of dimension $\operatorname{dim} F$. 
(c) Use (b) and the fact that the join operation is distributive over the wedge operation to prove Theorem 5.5.2.

(d) Show that $\Delta_{\mathrm{m}}$ is sequentially Cohen-Macaulay if $\Delta$ is.

Theorem 5.5.4. Let $\Delta$ be a $G$-simplicial complex on vertex set $[n]$ and let $\mathbf{m}$ be an $n$-tuple of positive integers. If $G$ acts on the inflation $\Delta_{\mathbf{m}}$ and this action commutes with the deflating map, then for all $r \in \mathbb{Z}$,

$$
\tilde{H}_{r}\left(\Delta_{\mathbf{m}}\right) \cong_{G} \bigoplus_{F \in \Delta / G}\left(\tilde{H}_{|F|-1}\left(\langle F\rangle_{\mathbf{m}(F)}\right) \otimes \tilde{H}_{r-|F|}\left(l k_{\Delta} F\right)\right) \uparrow_{G_{F}}^{G},
$$

where $\langle F\rangle$ is the subcomplex generated by $F$ and $\mathbf{m}(F)$ is the subsequence $\left(m_{i_{1}}, \ldots, m_{i_{t}}\right)$ of $\mathbf{m}=\left(m_{1}, \ldots, m_{n}\right)$ for $F=\left\{i_{1}<\cdots<i_{t}\right\}$.

Shareshian [154] and Shareshian and Wachs [156] use Theorems 5.5.2 and 5.5.4, to derive information about the homology of the Quillen complex $\mathcal{A}_{p}\left(\mathfrak{S}_{n}\right)$ from a hypergraph version of the matching complex. Another application of Theorems 5.5.2 and 5.5.4 can be found in [131]. Here we discuss the original example that led to the general fiber theorem, namely the colored chessboard complex [201].

Recall from Section 2.4.3 that the chessboard complex $M_{m, n}$ is the collection of rook placements on an $m \times n$ chessboard such that there is at most one rook in each row and each column. This complex arose in the work of Garst [78] on Tits coset complexes, in the work of Živaljević and Vrećica [221] on the colored Tverberg problem, in the work of Reiner and Roberts [138] on Segre algebras, and in the work of Babson and Reiner [10] on generalizations of Coxeter complexes. Like the matching complex, the chessboard complex has been extensively studied in the literature; see the survey article of Wachs [200]. We state just a few of the results on chessboard complexes here.

Theorem 5.5.5. Let $1 \leq m \leq n$ and $\nu_{m, n}=\min \left\{m,\left\lfloor\frac{m+n+1}{3}\right\rfloor\right\}-1$.

(a) (Garst [78]) If $n \geq 2 m-1$ then $M_{m, n}$ is Cohen-Macaulay.

(b) (Björner, Lovász, Vrećica and Živaljević [35]) The complex $M_{m, n}$ is $\left(\nu_{m, n}-\right.$ 1)-connected.

(c) (Shareshian and Wachs [155]) For $n \leq 2 m-5$ and $m+n \equiv 1 \bmod 3$,

$$
\tilde{H}_{\nu_{m, n}}\left(M_{m, n} ; \mathbb{Z}\right) \cong \mathbb{Z}_{3}
$$

Part (a) follows from part (b), and Ziegler [217] gives an alternative proof of part (b) by establishing shellability of the $\nu_{m, n}$-skeleton of $M_{m, n}$. Part (c) is one case of a more general torsion result, whose proof uses a chessboard complex analog of Bouc's representation theoretic formula (2.4.5) (due to Garst [78] for top homology, and to Friedman and Hanlon [75] in general) to construct a basis for the vector space $\tilde{H}_{m-1}\left(M_{m, n}\right)$. This basis consists of fundamental cycles constructed from pairs of standard Young tableaux via a classical combinatorial algorithm known as the Knuth-Robinson-Schensted correspondence. We remark that Parts (b) and (c) have analogs for the matching complex [35] [47] [155].

Let $M_{m, n}^{r}$ be the simplicial complex of placements of colored rooks on an $m \times n$ chessboard such that there is at most one rook in each row and each column, and the colors come from the set $[r]$. The colored chessboard complex arose in Garst's work as a more general example of a Tits coset complex. One can easily see that $M_{m, n}^{r}$ is the $(r, r, \ldots, r)$-inflation of $M_{m, n}$. 
Exercise 5.5.6 (Wachs [201]). Use Theorems 5.5.2, 5.5.4, and 5.5.5 to obtain a colored version of Theorem 5.5.5, i.e. a generalization to $M_{m, n}^{r}$, where $r \geq 1$.

For open problems on the topology of chessboard complexes, matching complexes and related complexes, see [200] and [155]. 



\section{BIBLIOGRAPHY}

1. F. Ardila and C. Klivans, The Bergman complex of a matroid and phylogenetic trees, J. Combin. Theory (B), to appear.

2. D. Armstrong, Braid groups, clusters, and free probability: an outline from the AIM workshop, Jan. 2005, http://www.aimath.org/WWN/braidgroups/.

3. V.I. Arnol'd, Topological invariants of algebraic functions, Trans. Moscow Math. Soc. 21 (1970), 30-52.

4. M. Aschbacher and S. Smith, On Quillen's conjecture for the p-groups complex, Ann. of Math. 2 (1993), 473-529.

5. C. Athanasiadis, Decompositions and connectivity of matching and chessboard complexes, Discrete Compute. Geom. 31 (2004), 395-403.

6. C. Athanasiadis, T. Brady and C. Watt, Shellability of noncrossing partition lattices, preprint 2005.

7. C. Athanasiadis and V. Reiner, Noncrossing partitions for the group $D_{n}$, SIAM J. Discrete Math. 18 (2004), 397-417.

8. E. Babson, A. Björner, S. Linusson, J. Shareshian, and V. Welker, Complexes of not $i$-connected graphs, Topology 38 (1999), 271-299.

9. E. Babson and P. Hersh, Discrete Morse functions from lexicographic orders, Trans. AMS 357 (2005), 509-534.

10. E. Babson and V. Reiner, Coxeter-like complexes, Discrete Math. Theor. Comput. Sci. 6 (2004), 223-252.

11. K. Baclawski, Whitney numbers of geometric lattices, Advances in Math. 16 (1975), 125-138.

12. K. Baclawski, Cohen-Macaulay ordered sets, J. Algebra 63 (1980), 226-258.

13. K. Baclawski and A. Björner, Fixed points and complements in finite lattices, J. Combin. Theory (A) 30 (1981), 335-338.

14. H. Barcelo, On the action of the symmetric group on the free Lie algebra and the partition lattice, J. Combin. Theory (A) 55 (1990), 93-129.

15. M.M. Bayer and L.J. Billera, Generalized Dehn-Sommerville relations for polytopes, spheres and Eulerian partially ordered sets, Invent. Math. $\mathbf{7 9}$ (1985), 143-157.

16. N. Bergeron, A hyperoctahedral analogue of the free Lie algebra, J. Combin. Theory (A) $\mathbf{5 8}$ (1991), 256-278.

17. D. Bessis, The dual braid monoid, Annales Scientifiques de l'École Normale Suprieure, 36 (2003), 647-683. 
18. L.J. Billera, S. Holmes, and K. Vogtmann, Geometry of the space of phylogenetic trees, Advances in Appl. Math. 27 (2001), 733-767.

19. L.J. Billera and C.W. Lee, A proof of sufficiency of McMullen's conditions for f-vectors of simplicial complexes, J. Combin. Th. (A) 31 (1981), 237-255.

20. L.J. Billera and A.N. Myers, Shellability of interval orders, Order 15 (1998/99), 113-117.

21. A. Björner, Shellable and Cohen-Macaulay partially ordered sets, Trans. AMS 260 (1980), 159-183.

22. A. Björner, On the homology of geometric lattices, Alg. Univ. 14 (1982), $107-128$.

23. A. Björner, Posets, regular CW-complexes and Bruhat order, Europ. J. Combin. 5 (1984), 7-16.

24. A. Björner, Orderings of Coxeter groups, combinatorics and algebra (Boulder, Colo., 1983), Contemp. Math. 34 (1984), 175-195.

25. A. Björner, The Möbius function of subword order, in Invariant Theory and Tableaux, ed. D. Stanton, IMA Volumes in Math. and its Applic., Vol 19, Springer-Verlag, New York, 1990, 118-124.

26. A. Björner, The homology and shellability of matroids and geometric lattices, in Matroid Applications, ed. N. White, Cambridge University Press, 1992, pp. 226-283.

27. A. Björner, The Möbius function of factor order, Theoretical Computer Science 117 (1993), 91-98.

28. A. Björner, Subspace arrangements, First European Congress of Mathematics, Paris 1992, A. Joseph et al. (Eds.), Progress in Math., Vol. 119, Birkhäuser, 1994, pp. 321-370.

29. A. Björner, Topological Methods, Handbook of Combinatorics, R. Graham, M. Grötschel and L. Lovász, (Eds), North-Holland, Amsterdam, 1995, pp. 1819-1872.

30. A. Björner and F. Brenti, Combinatorics of Coxeter Groups, Graduate Texts in Mathematics, Vol. 231, Springer-Verlag, New York, 2005.

31. A. Björner and K. Eriksson, Extendable shellability for rank 3 matroid complexes, Discrete Math. 132 (1994), 373-376.

32. A. Björner, A. Garsia, and R. Stanley, An introduction to the theory of Cohen-Macaulay posets, in Ordered Sets (I. Rival, ed.), Reidel, Dordrecht/Boston/London, 1982, pp. 583-615.

33. A. Björner and L. Lovász, Linear decision trees, subspace arrangements and Möbius functions, J. AMS 7 (1994), 677-706.

34. A. Björner, L. Lovász and A. Yao, Linear decision trees: volume estimates and topological bounds, Proc. 24th ACM Symp. on Theory of Computing (May 1992), ACM Press, New York, 1992, 170-177.

35. A. Björner, L. Lovász, S.T. Vrećica and R.T. Živaljević, Chessboard complexes and matching complexes, J. London Math. Soc. 49 (1994), 25-39.

36. A. Björner and B.E. Sagan, Subspace arrangements of type $B_{n}$ and $D_{n}$, J. Algebraic Combin. 5 (1996), 291-314.

37. A. Björner and M.L. Wachs, Bruhat order of Coxeter groups and shellability, Advances in Math. 43 (1982), 87-100.

38. A. Björner and M.L. Wachs, On lexicographically shellable posets, Trans. AMS 277 (1983), 323-341. 
39. A. Björner and M.L. Wachs, Generalized quotients in Coxeter groups, Trans. AMS 308 (1988), 1-37.

40. A. Björner and M.L. Wachs, Nonpure shellable complexes and posets I, Trans. AMS 348 (1996), 1299-1327.

41. A. Björner and M.L. Wachs, Nonpure shellable complexes and posets II, Trans. AMS 349 (1997), 3945-3975.

42. A. Björner and M.L. Wachs, Geometrically constructed bases for homology of partition lattices of type $A, B$ and $D$, special issue in honor of Richard Stanley, Electron. J. Combin. 11 (2004/05), Research Paper 3, 26 pp.

43. A. Björner, M.L. Wachs and V. Welker, Poset fiber theorems, Trans. AMS 357 (2005), 1877-1899.

44. A. Björner, M.L. Wachs and V. Welker, On sequentially Cohen-Macaulay complexes and posets, in preparation.

45. A. Björner and V. Welker, The homology of "k-equal" manifolds and related partition lattices, Advances in Math. 110 (1995), 277-313.

46. J.M. Boardman, Homotopy structures and the language of trees, Algebraic topology (Wisconsin, 1970) Proc. Symp. Pure Math. 22 (1971), 37-58.

47. S. Bouc, Homologie de certains ensembles de 2-sous-groupes des groupes symétriques, J. Algebra 150 (1992), 158-186.

48. T. Brady, A partial order on the symmetric group and new $K(\pi, 1)$ 's for the braid groups, Advances in Math. 161 (2001), 20-40.

49. T. Brady and C. Watt, $K(\pi, 1)$ 's for Artin groups of finite type, Proceedings of the Conference on Geometric and Combinatorial Group Theory, Part I, Geom. Dedicata 94 (2002), 225-250.

50. A.E. Browdy The (co)homology of lattices of partitions with restricted block size, Ph.D. dissertation, University of Miami, 1996.

51. A. Browdy and M.L. Wachs, On the (co)homology of the lattice of partitions with restricted block size, in preparation.

52. H. Bruggesser and P. Mani, Shellable decompositions of cells and spheres, Math. Scand. 29 (1971), 197-205.

53. C.C. Cadogan, The Möbius function and connnected graphs, J. Combin. Theory (B) 11 (1971), 193-200.

54. A.R. Calderbank, P. Hanlon and R.W. Robinson, Partitions into even and odd block size and some unusual characters of the symmetric groups, Proc. London Math. Soc. (3) 53 (1986), 288-320.

55. A. Chakrabarti, S. Khot and Y. Shi, Evasiveness of subgraph containment and related properties, SIAM J. Comput. 31 (2001/02), 866-875.

56. G. Danaraj and V. Klee, Shellings of spheres and polytopes, Duke Math. J. 41 (1974), 443-451.

57. C. de Concini and V. Lakshmibai, Arithmetic Cohen-Macaulayness and arithmetic normality for Schubert varieties, Amer. J. Math. 103 (1981), 835-850.

58. C. de Concini and C. Procesi, Wonderful models of subspace arrangements, Selecta Math., New Series 1 (1995), 459-494.

59. J. Désarménian, Une autre interprétation du nombre de dérangements, Sém. Lotharing. Combin. 9 (1984), 11-16.

60. J. Désarménian and M.L. Wachs, Descentes des derangements et mot circulaires, Sém Lotharing. Combin. 19 (1988), 13-21. 
61. X. Dong, Topology of bounded-degree graph complexes, J. Algebra 262 (2003), $287-312$.

62. X. Dong and M.L. Wachs, Combinatorial Laplacian of the matching complex, Electron. J. Combin. 9 (2002), R17.

63. J. G. Dumas, F. Heckenbach, D. Saunders and V. Welker, Computing simplicial homology based on efficient Smith normal form algorithms, Algebra, Geometry, and Software Systems, 177-206, Springer, Berlin, 2003.

64. A.M. Duval, Algebraic shifting and sequentially Cohen-Macaulay simplicial complexes, Electron. J. Combin. 3 (1996).

65. M.J. Dyer, Hecke algebras and shellings of Bruhat intervals, Compositio Math. 89 (1993), 91-115.

66. J.A. Eagon and V. Reiner, Resolutions of Stanley-Reisner rings and Alexander duality, J. Pure and Appl. Algebra 130 (1998), 265-275.

67. P.H. Edelman, The Bruhat order of the symmetric group is lexicographically shellable, Proc. AMS 82 (1981), 355-358.

68. F.D. Farmer, Cellular homology for posets, Math. Japonica 23 (1979), 607613.

69. E.M. Feichtner and G.M. Ziegler, On cohomology algebras of complex subspace arrangements, Trans. AMS 352 (2000), 3523-3555.

70. J. Folkman, The homology groups of a lattice, J. Math. Mech. 15 (1966), 631-636.

71. S. Fomin and N. Reading, Root systems and generalized associahedra, PCMI Lecture Notes, 2004.

72. R. Forman, Morse theory for cell complexes, Advances in Math. 134 (1998), 90-145.

73. R. Forman, Morse theory and evasiveness, Combinatorica 20 (2000), 498-504.

74. R. Forman, Discrete Morse theory, PCMI Lecture Notes, 2004.

75. J. Friedman and P. Hanlon, On the Betti numbers of chessboard complexes, J. Algebraic Combin. 8 (1998), 193-203.

76. W. Fulton, Young Tableaux, with Applications to Representation Theory and Geometry, LMS Student Texts 35, Cambridge University Press, Cambridge, 1997.

77. A.M. Garsia, Combinatorial methods in the theory of Cohen-Macaulay rings, Advances in Math. 38 (1980), 229-266.

78. P.F. Garst, Cohen-Macaulay complexes and group actions, Ph.D. Thesis, University of Wisconsin-Madison, 1979.

79. E. Getzler, and M.M. Kapranov, Cyclic operads and cyclic homology in Geometry, Topology and Physics, International Press, Cambridge, MA, 1995, pp. $167-201$.

80. M. Goresky and R.D. MacPherson Stratified Morse theory, Ergebnisse der Mathematic und ihrer Grenzgebiete 14, Springer-Verlag, 1988.

81. E. Gottlieb, Cohomology of Dowling lattices and Lie (super)algebras, Ph.D. Thesis, University of Miami, 1998.

82. E. Gottlieb, On the homology of the $h, k$-equal Dowling lattice, SIAM J. Discrete Math. 17 (2003), 50-71.

83. E. Gottlieb and M. L. Wachs, Cohomology of Dowling lattices and Lie (super)algebras, Advances in Appl. Math. 24 (2000), 301-336. 
84. H.T. Hall, Counterexamples in Discrete Geometry, Ph. D. Thesis, University of California, Berkeley, 2004.

85. P. Hanlon, The fixed-point partition lattices, Pacific J. Math. 96 (1981), 319341.

86. P. Hanlon, The characters of the wreath product group acting on the homology groups of the Dowling lattices, J. Algebra 91 (1984), 430-463.

87. P. Hanlon, Otter's method and the homology of homeomorphically irreducible $k$-trees, Jour. Combin. Theory (A), 74 (1996), 301-320.

88. P. Hanlon and P. Hersh, Multiplicity of the trivial representation in rankselected homology of the partition lattice, J. Algebra 266 (2003), 521-538.

89. P. Hanlon and P. Hersh, A hodge decomposition for the complex of injective words, Pacific J. Math. 214 (2004), 109-125.

90. P. Hanlon and R.P. Stanley, A q-deformation of a trivial symmetric group action, Trans. AMS 350 (1998), 4445-4459.

91. P. Hanlon and M.L. Wachs, On Lie k-algebras, Advances in Math. 113 (1995), 206-236.

92. A. Hatcher, Algebraic Topology, Cambridge University Press, 2001.

93. P. Hersh, Lexicographic shellability for balanced complexes, J. Algebraic Combin. 17 (2003), 225-254.

94. J. Herzog and T. Hibi, Componentwise linear ideals, Nagoya Math. J. 153 (1999), 141153.

95. J. Herzog, V. Reiner, and V. Welker, Componentwise linear ideals and Golod rings, Michigan Math. J. 46 (1999), 211-223.

96. M. Hochster, Rings of invariants of tori, Cohen-Macaulay rings generated by monomials, and polytopes, Annals of Math. 96 (1972), 318-337.

97. A. Hultman, Lexicographic shellability and quotient complexes, J. Algebraic Combin. 16 (2002), 83-86.

98. A. Hultman, The combinatorics of twisted involutions in Coxeter groups, Trans. AMS, to appear.

99. F. Incitti, The Bruhat order on the involutions of the symmetric group, J. Algebraic Combin. 20 (2004), 243-261.

100. F. Incitti, The Bruhat order on the involutions of the hyperoctahedral group, Europ. J. Combin. 24 (2003), 825-848.

101. F. Incitti, Bruhat order on the involutions of classical Weyl groups, Advances in Appl. Math., to appear.

102. J. Jonsson, On the topology of simplicial complexes related to 3-connected and Hamiltonian graphs, J. Combin. Theory (A) 104 (2003), 169-199.

103. J. Jonsson, Simplicial Complexes of Graphs, Ph.D. thesis, Royal Institute of Technology, Sweden, 2005.

104. A. Joyal, Foncteurs analytiques et espèces de structure, Springer Lecture Notes, No. 1234, Springer-Verlag, Berlin, Heidelberg, New York, 1986.

105. D.B. Karaguezian, V. Reiner and M.L. Wachs, Matching complexes, bounded degree graph complexes, and weight spaces of $G L_{n}$-complexes, J. Algebra 239 (2001), 77-92.

106. J. Kahn, M. Saks and D. Sturtevant, A topological approach to evasiveness, Combinatorica 4 (1984), 297-306.

107. B. Kind and P. Kleinschmidt, Schälbare Cohen-Macauley-Komplexe und ihre Parametrisierung, Math. Z. 167 (1979), 173-179. 
108. A.A. Klyachko, Lie elements in the tensor algebra, Siberian Math. J. 15 (1971), 1296-1304.

109. M. Kontsevich, Formal (non)-commutative symplectic geometry, in the Gelfand Mathematical Seminar, 1990-1992, eds L. Corwin et al., Birkhäuser, Boston, 1993, pp. 173-187.

110. D. Kozlov, General lexicographic shellability and orbit arrangements, Ann. of Combin. 1 (1997), 67-90.

111. D. Kozlov, Rational homology of spaces of complex monic polynomials with multiple roots, Mathematika 49 (2002), 77-91.

112. C. Kratzer and J. Thévenaz, Type d'homotopie des treillis et treillis des sousgroupes d'un groupe fini, Comment. Math. Helv. 60 (1985), 86-106.

113. G. Kreweras, Sur les partitions non croisées d'un cycle, Discrete Math. 1 (1972), 333-350.

114. R. Ksontini, Propriétés homotopiques du complexe de Quillen du groupe symétrique, Thèse de Doctorate, Université de Lausanne, 2000.

115. R. Ksontini, Simple connectivity of the Quillen complex of the symmetric group, J. Combin. Theory (A) 103 (2003), 257-279.

116. R. Ksontini, The fundamental group of the Quillen complex of the symmetric group, J. Algebra 282 (2004), 33-57.

117. G. Lehrer and L. Solomon, On the action of the symmetric group on the cohomology of the complement of its reflecting hyperplanes, J. Algebra 104 (1986), 410-424.

118. W.B. Lickorish, Unshellable triangulations of spheres, European J. Combin. 12 (1991) 527-530.

119. S. Linusson, Partitions with restricted block sizes, Möbius functions, and the k-of-each problem, SIAM J. Discrete Math. 10 (1997), 18-29.

120. S. Linusson, J. Shareshian and V. Welker, Complexes of graphs with bounded matching size, preprint 2003.

121. D.E. Littlewood, The Theory of Group Characters and Matrix Representations of Groups, 2nd ed., Oxford University Press, 1950.

122. G. Lusztig, Total positivity in partial flag manifolds, Representation Theory, 2 (1998), 70-78.

123. F.H. Lutz, Small examples of nonconstructible simplicial balls and spheres, SIAM J. Discrete Math. 18 (2004), 103-109.

124. I.G. Macdonald, Symmetric Functions and Hall Polynomials, Oxford Mathematical Monographs, Oxford University Press, New York, 1979.

125. J. McCammond, Noncrossing partitions in surprising locations, Amer. Math. Monthly, to appear.

126. P. McMullen, The maximum number of faces of a convex polytope, Mathematika 17 (1970), 179-184.

127. E. Miller and B. Sturmfels, Combinatorial Commutative Algebra, Grad. Texts in Math., Springer-Verlag, New York 2004.

128. J. Munkres, Topological results in combinatorics, Michigan Math. J. 31 (1984), 113-128.

129. P. Orlik and L. Solomon, Combinatorics and topology of complements of hyperplanes, Invent. Math. 56 (1980), 167-189.

130. P. Orlik and H. Terao, Arrangements of Hyperplanes, Grundlehren Series 300, Springer Verlag, Berlin-Heidelberg. 
131. J. Pakianathan and E. Yalçin, On commuting and non-commuting complexes, J. Algebra 236 (2001), 396-418.

132. R. Proctor, Classical Bruhat orders and lexicographically shellability, J. Algebra 77 (1982), 104-126.

133. J.S. Provan and L.J. Billera, Decompositions of simplicial complexes related to diameters of convex polyhedra, Math. Oper. Res. 5 (1980), 576-594.

134. P. Pudlák and J. Tuma, Every finite lattice can be embedded in a finite partition lattice, Alg. Univ. 10 (1980), 74-95.

135. D. Quillen, Homotopy properties of the poset of non-trivial p-subgroups of a group, Advances in Math. 28 (1978), 101-128.

136. M. Readdy, The pre-WDVV ring of physics and its topology, Ramanujan J., Special issue on the Number Theory and Combinatorics in Physics, to appear.

137. V. Reiner, Non-crossing partitions for classical reflection groups, Discrete Math. 177 (1997), 195-222.

138. V. Reiner and J. Roberts, Minimal resolutions and homology of chessboard and matching complexes, J. Algebraic Combin. 11 (2000), 135-154.

139. V. Reiner and M.L. Wachs, A decomposition of the eigenspaces of the random to top card shuffling operator, in preparation.

140. V. Reiner and P. Webb, The combinatorics of the bar resolution in group cohomology, J. Pure Appl. Algebra 190 (2004), 291-327.

141. V. Reiner and V. Welker, A homological lower bound for order dimension of lattices, Order 16 (1999), 165-170.

142. G. Reisner, Cohen-Macaulay quotients of polynomial rings, Advances in Math. 21 (1976), 30-49.

143. R.W. Richardson and T.A. Springer, The Bruhat order on symmetric varieties, Geom. Dedicata 49 (1990), 389-436.

144. K. Rietsch, Total positivity and real flag varieties, Ph.D. Thesis, MIT, 1998.

145. A. Robinson, Partition complexes, duality and integral tree representations, Algebr. Geom. Topol. 4 (2004), 943-960.

146. C.A. Robinson and S. Whitehouse The tree representation of $\sum_{n+1}$, J. Pure and App. Algebra 111 (1996), 245-253.

147. G.-C. Rota, On the foundations of combinatorial theory, I. Theory of Möbius functions, Z. Wahrscheinlichkeitstheorie und Verw. Gebiete 2 (1964), 340368.

148. B.E. Sagan, Shellability of exponential structures, Order 3 (1986), 47-54.

149. B.E. Sagan, The Symmetric Group. Representations, Combinatorial Algorithms, and Symmetric Functions, second edition, Graduate Texts in Mathematics, 203, Springer-Verlag, New York, 2001.

150. L. Schläfli, Theorie der vielfachen Kontiutät, written 1850-1852; Zürcher und Furrer, Zürich 1901; Denkschriften der Schweizerischen naturforschenden Gesellschaft 38 (1901), 1-237.

151. J. Shareshian, On the shellability of the order complex of the subgroup lattice of a finite group, Trans. AMS 353 (2001), 2689-2703.

152. J. Shareshian, Discrete Morse theory for complexes of 2-connected graphs, Topology 40 (2001), 681-701.

153. J. Shareshian, Topology of order complexes of intervals in subgroup lattices, J. Algebra 268 (2003), 677-686. 
154. J. Shareshian, Hypergraph matching complexes and Quillen complexes of symmetric groups, J. Combin. Theory (A) 106 (2004), 299-314.

155. J. Shareshian and M.L. Wachs, Torsion in the matching complex and chessboard complex, Advances in Math., to appear.

156. J. Shareshian and M.L. Wachs, Top homology of hypergraph matching complexes, p-cycle complexes and Quillen complexes of symmetric groups, in preparation.

157. J. Shareshian and M.L. Wachs, On the $1 \bmod k$ partition poset and graph complexes, in preparation.

158. R. Simion, On q-analogues of partially ordered sets, J. Combin. Theory (A) 72 (1995), 135-183.

159. R. Simion, Noncrossing partitions, Discrete Math. 217 (2000), 367-409.

160. R.S. Simon, Combinatorial properties of "cleanness", J. Algebra 167 (1994), $361-38$.

161. L. Solomon, A decomposition of the group algebra of a finite Coxeter group, J. Algebra 9 (1968), 220-239.

162. R.P. Stanley, Ordered structures and partitions, Memoirs AMS no. 119 (1972).

163. R.P. Stanley, Supersolvable lattices, Alg. Univ. 2 (1972), 197-217.

164. R.P. Stanley, Finite lattices and Jordan-Hölder sets, Alg. Univ. 4 (1974), 361-371.

165. R.P. Stanley, Exponential structures, Stud. Appl. Math. 59 (1978), 73-82.

166. R.P. Stanley, The number of faces of a simplicial convex polytope, Advances in Math. 35 (1980), 236-238.

167. R.P. Stanley, Some aspects of groups acting on finite posets, J. Combin. Theory (A) 32 (1982), 132-161.

168. R.P. Stanley, Combinatorics and Commutative Algebra, Second Edition, Progress in Mathematics 41, Birkhäuser, Boston 1983, 2nd ed. 1996.

169. R.P. Stanley, Enumerative Combinatorics, Vol. 1, Wadsworth \& Brooks/Cole, Monterey, CA, 1986, Second edition, Birkhäuser, Boston, 1995.

170. R.P. Stanley, Flag-symmetric and locally rank-symmetric partially ordered sets, Foata Festschrift, Electron. J. Combin. 3 (1996), RP 6, 22 pp.

171. R.P. Stanley, Parking functions and noncrossing partitions, Wilf Festschrift, Electron. J. Combin. 4 (1997), RP 20, 14 pp.

172. R.P. Stanley, Enumerative Combinatorics, Vol. 2, Cambridge Studies in Advanced Mathematics 62, Cambridge University Press, Cambridge, 1999.

173. R.P. Stanley Recent developments in algebraic combinatorics, Israel J. of Math. 143 (2004), 317-339.

174. R.P. Stanley, An introduction to hyperplane arrangements, PCMI Lecture Notes, 2004.

175. S. Sundaram, The homology representations of the symmetric group on Cohen-Macaulay subposets of the partition lattice, Advances in Math. 104 (1994), 225-296.

176. S. Sundaram, Applications of the Hopf trace formula to computing homology representations, Jerusalem combinatorics '93, 277-309, Contemp. Math., 178, Amer. Math. Soc., Providence, RI, 1994.

177. S. Sundaram, The homology of partitions with an even number of blocks, J. Algebra 4 (1995). 69-92. 
178. S. Sundaram, Plethysm, partitions with an even number of blocks and Euler numbers, in "Formal Power Series and Algebraic Combinatorics 1994", 171-198, DIMACS Series in Discrete Mathematics and Theoretical Computer Science 24, Amer. Math. Soc., Providence, RI, 1996.

179. S. Sundaram, Homotopy of non-modular partitions and the Whitehouse module, J. Algebraic Combin. 9 (1999), 251-269.

180. S. Sundaram, On the topology of two partition posets with forbidden block sizes, J. Pure Appl. Algebra 155 (2001), 271-304.

181. S. Sundaram, A homotopy equivalence for partition posets related to liftings of $S_{n-1}$-modules to $S_{n}$, J. Combin. Theory (A) 94 (2001), 156-168.

182. S. Sundaram and M.L. Wachs, The homology representations of the $k$-equal partition lattice, Trans. AMS 349 (1997), 935-954.

183. S. Sundaram and V. Welker, Group representations on the homology of products of posets, J. Combin. Theory (A) 73 (1996), 174-180.

184. S. Sundaram and V. Welker, Group actions on arrangements and applications to configuration spaces, Trans. AMS 349 (1997), 1389-1420.

185. E. Swartz, g-Elements, finite buildings and higher Cohen-Macaulay connectivity, preprint 2005.

186. G. Sylvester, Continuous spin ising ferromagnets, Ph.D. Thesis, MIT, 1976.

187. J. Thévenaz and P.J. Webb, Homotopy equivalence of posets with a group action, J. Combin. Theory (A) 56 (1991), 173-181.

188. H. Trappmann and G. Ziegler, Shellability of complexes of trees, J. Combin. Theory (A) 82 (1998), 168-178.

189. W. Trotter, Combinatorics and Partially Ordered Sets: Dimension Theory, Johns Hopkins Series in the Mathematical Sciences, Johns Hopkins University, Press, Baltimore, 1992.

190. V. Turchin, Homologies of complexes of doubly connected graphs, Russian Mathematical Surveys (Uspekhi) 52 (1997), 426-427.

191. V.A. Vassiliev, Complexes of connected graphs in the Gelfand Mathematical Seminar, 1990-1992, eds. L. Corwin et al., Birkhäuser, Boston, 1993, pp. 223-235.

192. V.A. Vassiliev, Complements of Discriminants of Smooth Maps: Topology and Applications, Revised Edition, Vol. 98 of Translations of Mathematical Monographs., Amer. Math. Soc., Providence, RI, 1994.

193. V.A. Vassiliev, Topology of two-connected graphs and homology of spaces of knots, in: S. L. Tabachnikov (ed.), "Differential and Symplectic Topology of Knots and Curves", AMS Transl. Ser. 2, 190, Amer. Math. Soc., Providence, RI, 1999.

194. A. Vince and M.L. Wachs, A shellable poset that is not lexicographically shellable, Combinatorica 5 (1985), 257-260.

195. K. Vogtmann, Local Structure of some out $\left(F_{n}\right)$-complexes, Proc. Edinburgh Math. Soc. (2) 33 (1990), 367-379.

196. M. L. Wachs, A basis for the homology of the d-divisible partition lattice, Advances in Math. 117 (1996), 294-318.

197. M. L. Wachs, On the (co)homology of the partition lattice and the free Lie algebra, Special issue in honor of Adriano Garsia, Discrete Math. 193 (1998), 287-319. 
198. M. L. Wachs, Obstructions to shellability, Discrete Compute. Geom. 22 (1999), 95-103.

199. M.L. Wachs, Whitney homology of semipure shellable posets, J. Algebraic Combin. 9 (1999), 173-207.

200. M.L. Wachs, Topology of matching, chessboard, and general bounded degree graph complexes, Special issue in memory of Gian-Carlo Rota, Algebra Universalis 49 (2003), 345-385.

201. M.L. Wachs, Bounded degree digraph and multigraph matching complexes, in preparation.

202. M.L. Wachs, Poset fiber theorems and Dowling lattices, in preparation.

203. M.L. Wachs and J.W. Walker, On geometric semilattices, Order 2 (1986), 367-385.

204. J.W. Walker, Topology and Combinatorics of Ordered Sets, MIT Ph.D. thesis, 1981.

205. J.W. Walker, Canonical homeomorphisms of posets, European J. Combin. 9 (1988), 97-107.

206. J.W. Walker, A poset which is shellable but not lexicographically shellable, European J. Combin. 6 (1985), 287-288.

207. P. J. Webb, Subgroup complexes, Arcata Conference on Representations of Finite Groups (Arcata, Calif., 1986), 349-365, Proc. Sympos. Pure Math., 47, Part 1, Amer. Math. Soc., Providence, RI, 1987.

208. V. Welker, G.M. Ziegler and R.T. Živaljević, Homotopy colimits - comparison lemmas for combinatorial applications, J. Reine Angew. Mathematik (Crelles Journal) 509 (1999), 117-149.

209. S. Whitehouse, The Eulerian representations of $\sum_{n}$ as restrictions of representations of $\sum_{n+1}$, J. Pure Appl. Algebra 115 (1996), 309-321.

210. L.K. Williams, Shelling totally nonnegative flag varieties, preprint 2005.

211. A. Yao, Monotone bipartite graph properties are evasive, SIAM J. Comput. 17 (1988), 517-520.

212. S. Yuzvinsky, Small rational model of subspace complement, Trans. AMS 354 (2002), 1921-1945.

213. T. Zaslavsky, Facing up to arrangements: Face-count formulas for partitions of space by hyperplanes, Memoirs AMS 154 (1975).

214. G.M. Ziegler, Combinatorial models for subspace arrangements, HabilitationsSchrift, Techn. Univ., Berlin 1992.

215. G.M. Ziegler, Matroid shellability, $\beta$-systems, and affine hyperplane arrangements, J. Algebraic Combin. 1 (1992), 283-300.

216. G.M. Ziegler, On the difference between real and complex arrangements, Math. Zeitschrift 212 (1993), 1-11.

217. G.M. Ziegler, Shellability of chessboard complexes, Israel J. Math. 87 (1994), 97-110.

218. G.M. Ziegler, Lectures on Polytopes, Grad. Texts in Math. 152, SpringerVerlag, New York 1995.

219. G.M. Ziegler, Shelling polyhedral 3-balls and 4-polytopes, Discrete Compute. Geom. 19 (1998), 159-174.

220. G.M. Ziegler and R.T. Živaljević, Homotopy type of arrangements via diagrams of spaces, Math. Ann. 295 (1993), 527-548. 
221. R.T. Živaljević and S.T. Vrećica, The colored Tverberg problem and complexes of injective functions, J. Combin. Theory (A) 61 (1992), 309-318. 